\title{
FLORISTIC SURVEY OF VASCULAR PLANTS IN CRAWFORD AND CHEROKEE COUNTIES IN SOUTHEASTERN KANSAS, U.S.A.
}

\author{
Samantha Young Pryer \\ Department of Biology \\ Pittsburg State University \\ Pittsburg, Kansas 66762, U.S.A. \\ spryer@ufl.edu
}

\author{
Neil Snow \\ Department of Biology \\ Pittsburg State University \\ Pittsburg, Kansas 66762, U.S.A. \\ nsnow@pittstate.edu
}

\author{
John Kartesz \\ Biota of North America Program \\ 9319 Bracken Lane \\ Chapel Hill, North Carolina 27516, U.S.A. \\ jkartesz@bonap.org
}

\begin{abstract}
This study documented the vascular flora of Crawford and Cherokee counties in southeastern Kansas. Each county experienced limited collecting in recent decades. A Pittsburg State University (PSU) student (Earl Sam Gibson) summarized the flora for Crawford County in 1963, but an extensive survey had never been done for Cherokee County. Fieldwork for the present study occurred mostly in 2014 and 2015. Based on approximately 6450 newly collected specimens, vouchers at PSU (including those of Gibson), and taxa documented by Biota of North America (BONAP) (Kartesz 2019), a total of 1420 unique taxa (species, subspecies and varieties) are reported for both counties combined, including 148 families, 591 genera, and 111 non-nominal infraspecific taxa. Of these, 1177 (82.9\%) are native and 243 (17.1\%) are non-native. A total of 44 state and 233 county records are documented. Included were 15 species of hybrid origin and 22 that are adventive from other parts of North America. Significant among the state records is the first published adventive occurrence of the genus Pinus in Kansas. Cherokee County has 1281 taxa (including 81 county records), whereas Crawford County includes 1139 taxa (and 152 county records). An important finding of our study, along with those of other recent floristic studies, is that vascular plant distributions at the state and county levels in the U.S.A. are less well documented than probably assumed. This is especially true for weedy taxa occurring in highly agricultural and industrial counties, as well as taxa with small, inconspicuous flowers and inflorescences, and counties relatively distant from universities. Undocumented occurrences of such taxa may adversely affect interpretations and distribution patterns of abundance, as for example when modeling plant distributions.
\end{abstract}

\section{CHINESE ABSTRACT}

该研究对堪萨斯州东南部克劳福德县和切罗基县的维管植物区系进行了分析。近几十年来, 在克劳福德县和切罗基县仅进行过有限的植物 采集, 一位匹兹堡州立大学的学生 (ES GIBSON) 早在1963年对克劳福德县的植物群进行了分析, 但从未有人在切罗基县进行过广泛的调查。 这项研究的调查时间为 2014 年和 2015 年。在约 6450 个新采集的标本以及吉布森研究的成果和北美生物群的植物群记录 (BONAP) (KARTESZ 2019）的基础上, 两个县总共记录了 1420 个独立分类群 (物种, 亚种和品种), 其中 148 科, 591属和111种非典型种内分类群。其中有 1177 (82.9\% ) 是本地种, 243 (17.1\%) 是非本地种。此项研究记录了来自44个州和 233 个县的记录, 远远超出研究预期。其中包括 15 种来源于杂交的种, 22 种来自北美其他地区的外来物种。在堪萨斯州Pinus外来属的首次发现具有重要意义。切罗基县有 1281 个分类群 (包括 81 个县的记录), 而克 劳福德县共有1139个分类群 (包括152个县的记录)。此项研究的一个重要发现, 包括其他近期植物区系研究的重要发现是, 美国州和县级的 维管植物分布记录比预计的要不完善许多, 尤其是对于在高度农业和工业县中分布广泛的杂草分类群以及具有小型花和花序的分类群, 还有 距离大学较远的区域。对于这种类群的不完善记录可能会对丰富度和分布模式产生不利影响, 例如在对植物分的布进行建模时。

\section{RESUMEN}

Este estudio documenta la flora vascular de los condados de Crawford y Cherokee en el sureste de Kansas. Todos los condados tuvieron colectas limitadas en las décadas recientes. Un estudiante de la Pittsburg State University (PSU) (Earl Sam Gibson) resumió la flora del condado de Crawford en 1963, pero nunca se hizo un estudio extensivo en el condado de Cherokee. El trabajo de campo del presente estudio se realizó mayormente en 2014 y 2015. Basados en unos 6450 especímenes colectados, cuyos testigos están en PSU (incluidos los de Gibson), y taxa documentados por Biota of North America (BONAP) (Kartesz 2019), se cita un total de 1420 taxa (especies, subespecies y variedades) para ambos condados combinados, incluidos en 148 familias, 591 géneros, y 111 taxa infraspecíficos no -nominales. De estos, 1177 (82.9\%) son nativos y 243 (17.1\%) son no nativos. Se documenta un total de 44 citas para el estado y 233 para el condado. Se incluyen 15 especies de 
origen híbrido y 22 que son adventicias de otras partes de Norte América. Significantes entre las citas para el estado es la primera ocurrencia publicada de una especie del género Pinus en Kansas. El condado de Cherokee tiene 1281 taxa (incluye 81 citas del condado), mientras el condado de Crawford incluye 1139 taxa (y 152 citas para el condado). Un hallazgo importante de nuestro estudio, junto con otros estudios florísticos recientes, es que las distribuciones de plantas vasculares a los niveles de estado y condado en U.S.A. están menos documentados de lo que se asumía. Esto es especialmente cierto para las malas hierbas que aparecen en condados altamente agriculturales e industriales, así como taxa con flores pequeñas e inflorescencias, inconspicuas, y condados relativamente alejados de universidades. Ocurrencias no documentadas de tales taxa pueden afectar adversamente las interpretaciones y patrones de distribución de la abundancia, como por ejemplo cuando se modelizan las distribuciones de plantas.

\section{INTRODUCTION}

Crawford and Cherokee counties are located in the southeastern corner of Kansas (Fig. 1). Of roughly equal sizes, Crawford County covers approximately $1541 \mathrm{~km}^{2}\left(595 \mathrm{mi}^{2}\right.$ ) and Cherokee County ca. $1533 \mathrm{~km}^{2}$ (ca. 592 $\mathrm{mi}^{2}$ ), totaling ca. $3074 \mathrm{~km}^{2}\left(1187 \mathrm{mi}^{2}\right)$. Both have relatively diverse vascular floras among counties in Kansas, including approximately half of the known vascular plant flora for the entire state (Kartesz 2019).

Both counties have experienced sporadic and occasionally more focused collecting. For example, working from the University of Kansas, H.A. Stephens collected over 90,000 specimens across the Great Plains in the 1960s and 70s, including southeastern Kansas, which were summarized in Atlas of the Flora of the Great Plains (Barker \& Barkley 1977) and later in Flora of The Great Plains (GPFA 1986). Various projects by the Kansas Biological Survey have evaluated smaller parcels in these counties (see Pryer 2018), and a few masters' theses from Pittsburg State University studied plant diversity in Crawford County, always on small areas and typically from a perspective of plant succession (Bass 1949; Featherstone 1952; Nelson 1952).

The only county-wide survey of these counties was that of Earl Sam Gibson (1939-2011), who surveyed the entirety of Crawford County nearly sixty years ago (Gibson 1963). (Gibson eventually taught at Missouri Southern State University [then Missouri Southern College] in Joplin, eventually becoming the Chair of the Department of Biology.) Distributional data of plant taxa from the earlier MS theses (Bass 1949; Featherstone 1952; Nelson 1952) at PSU were incorporated by Gibson (1963). However, no extensive floristic surveys had ever been done for Cherokee County.

Bordered by Missouri to the east and Oklahoma to the south, this region lies within the ecotone between the eastern deciduous forest and tallgrass prairie. The landscape is underpinned by a geology that transitions significantly moving from south to north. The Ozark Plateau in the southeastern corner in Cherokee County contains the region's oldest bedrock; a Mississippian limestone overtopped by mixed deciduous oak hickory forests. The remainder of Cherokee County and about half of Crawford County is underlain by Pennsylvanian bedrock in the Cherokee lowlands, where old surface coal mines are prevalent alongside the prairies. Running diagonally, across the northeast to southwest half of Crawford County are the Osage Cuestas. These are parallel ridges comprised of gentle slopes on the west side and steeper ones to the east; alternating between shale and limestone (Buchanan 2010). Lying between different ecosystems, and having the state's highest average annual precipitation, it is not surprising that this area of Kansas supports a diverse flora.

The first attempt at documenting the flora of southeastern Kansas was by Leland J. Gier (1931) in his publication A Preliminary Key to the Herbaceous Dicotyledons of the Cherokee Strip of Southeast Kansas, which included only about 200 species. The Flora of Kansas (Gates 1940) and A Manual of the Flowering Plants of Kansas (Barkley 1968) were the first two extensive attempts at documenting the flora of the state. Twenty years later, the first edition of Flora of The Great Plains (GPFA 1986) was published, which included southeastern Kansas. In the most recent floristic work, the Kansas Biological Survey established a few natural area sites within Cherokee County in 2005 (Loring et al. 2005), but plant collecting was limited. A floristics survey by two PSU faculty members, Bennington Ross and Harry H. Hall, focused on "Crawford County State Park" (Ross \& Hall 1939); however, that area is now known as Mined Lands Wildlife Area No. 1, and the current Crawford County State Park is located farther north between Farlington and the southern border of Bourbon County. A master's thesis at Pittsburg State University study focused on the systematics of Vitaceae (Kirkpatrick 1973). Although not extensively collected, southeastern Kansas had experienced sporadic collections historically. These collections are housed in several herbaria, including the T.M. Sperry Herbarium, Kansas State 


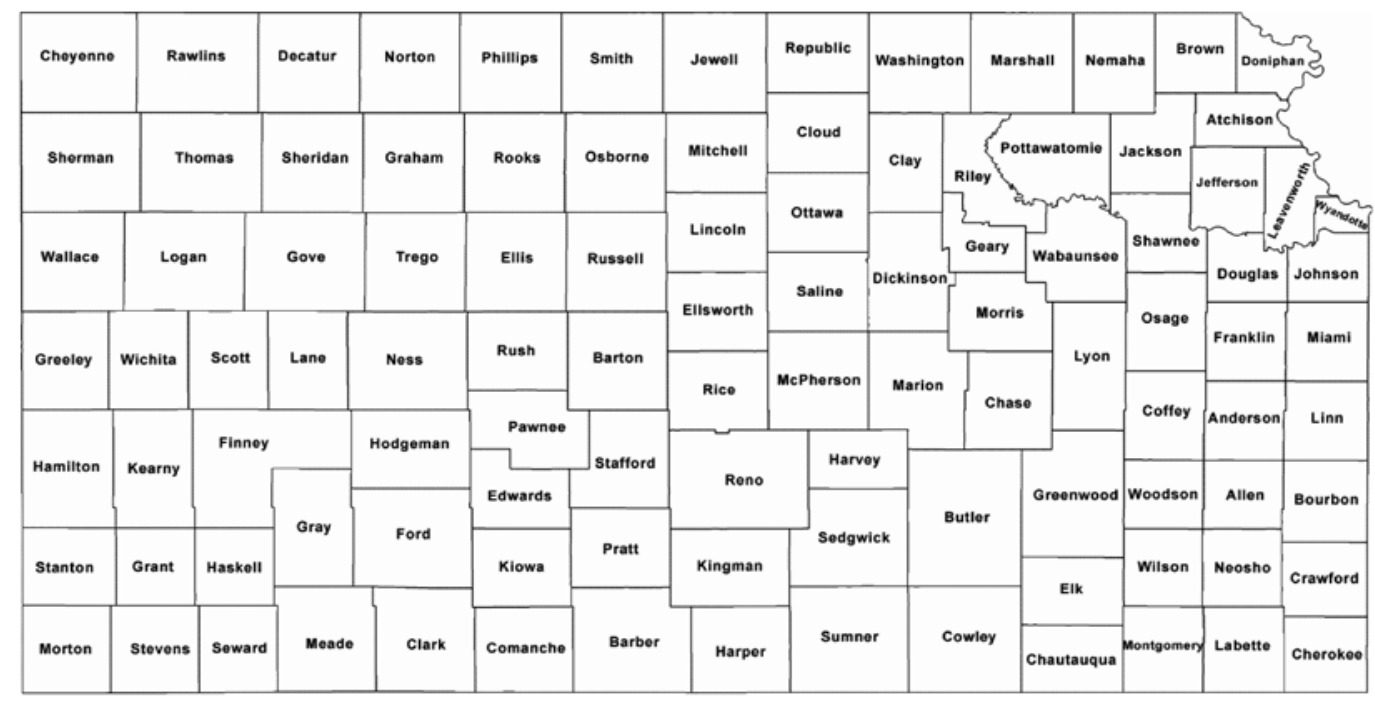

Get Printable Maps From:

WaterproofPaper.com

Fig. 1. Kansas County map. Crawford and Cherokee counties occupy the southeast corner of Kansas along the border of Missouri. Cherokee County is bordered by Oklahoma to the south.

University Herbarium, McGregor Herbarium (University of Kansas), H.A. Stevens Herbarium at Emporia State University, the Wichita State Herbarium, and Missouri Botanical Garden.

Climate.-Southeastern Kansas, located in the center of the U.S.A., has a humid continental climate. It is an area with considerable seasonal disparity of average temperatures. Summers tend to be hot and winters typically have one to several (and often protracted) spells of freezing weather. The region experiences weather extremes from severe thunderstorms, ice storms, and tornados.

Based on data obtained from the National Oceanic and Atmospheric Association (NOAA) weather station (GHCND:USC00141740) in Columbus, KS, the average annual precipitation for southeastern Kansas is approximately $117 \mathrm{~cm}(46 \mathrm{in})$, with an average temperature of $14^{\circ} \mathrm{C}\left(57^{\circ} \mathrm{F}\right)(\mathrm{NCDC})$. The temperature ranges from an average minimum of $-3.33^{\circ} \mathrm{C}\left(26^{\circ} \mathrm{F}\right)$ to an average maximum of $31^{\circ} \mathrm{C}\left(88^{\circ} \mathrm{F}\right)$. These averages summarize annual data between 1981-2010. Because of the relatively flat topography of the region, weather patterns vary minimally across the sampling area; thus only one weather station is cited.

Geography.-Lying within a transitional zone, southeastern Kansas comprises three main physiographic regions (Fig. 2). Cherokee County specifically represents a triangle of approximately $129 \mathrm{~km}^{2}$ of the Ozark Plateau (Buchanan 2010) in its southeastern corner, while the Cherokee Lowlands dominate the remainder of the county, extending through half of Crawford County (Buchanan 2010). The Osage Cuestas comprise much of northern half of Crawford County. Persisting strip pits from surface coal mining activities that terminated in the 1970s are prevalent throughout both counties (Buchanan 2010; Bailey \& Hooey 2017). On the map of Kansas published by the US Geological Survey, the Ozark Plateau is known as the Springfield Plateau, and the Cherokee Lowlands as the Cherokee Plains (Chapman et al. 2001).

Ozark plateau: The small occurrence of the Ozark Plateau in southeastern Cherokee County represents the oldest sediment exposure in Kansas. It was formed in the Mississippian era, about 320-360 million years ago (Fig. 2) (Buchanan 2010). At that time, seas covered much of the land areas in this region and carved tunnels into the underlain bedrock. The Ozark Plateau bedrock, comprised primarily of a soft limestone and embedded with harder chert and flint deposits, eroded over time, leaving most of the chert and flint deposits 
Generalized Physiographic Map of Kansis

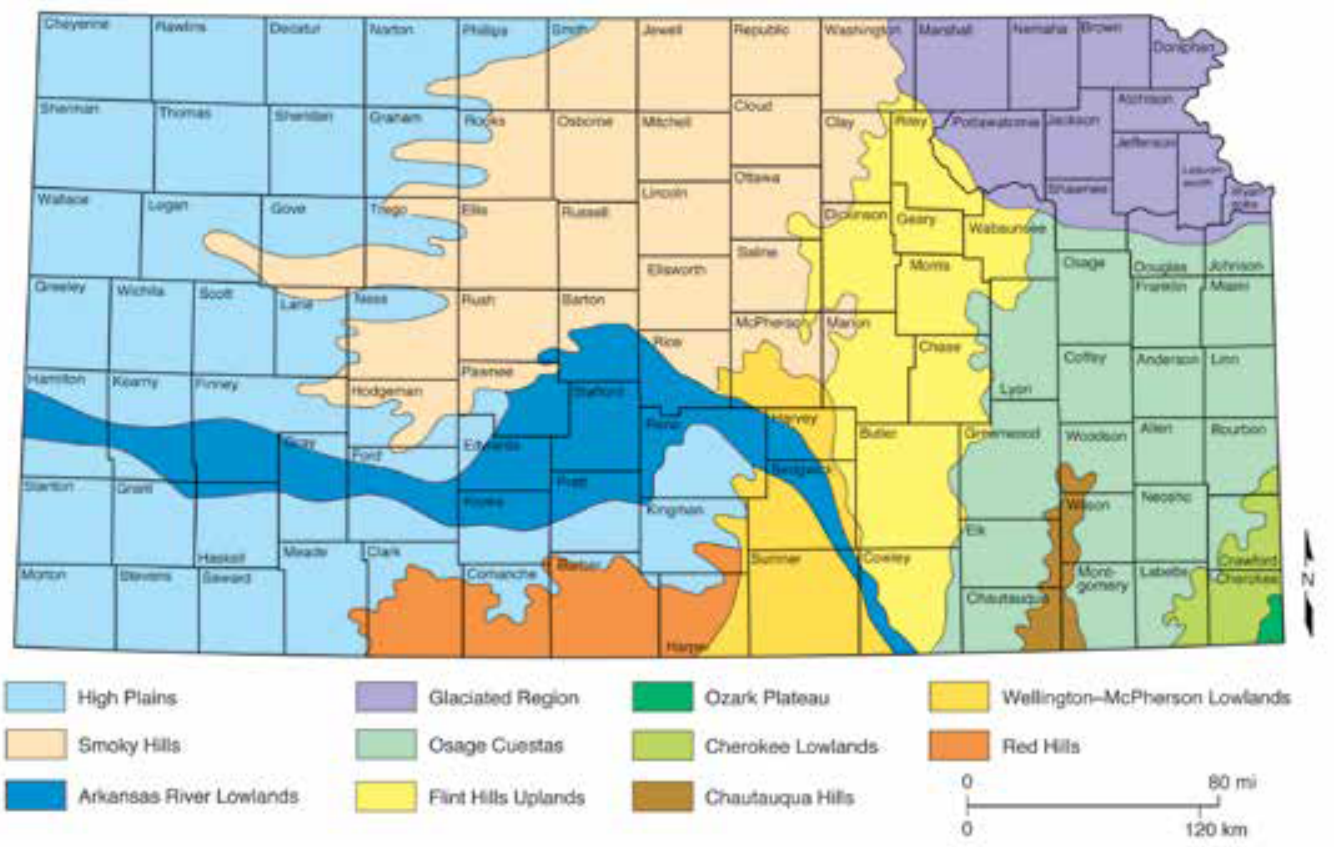

FIG. 2. Physiographic map of Kansas (Kansas Geological Survey).

intact. Higher elevations in this area are covered with this weathered cherty limestone gravels and various soil types (Buchanan 2010).

Cherokee lowlands: The Cherokee Lowlands, comprised of Pennsylvanian shale and sandstone bedrocks, dominate most of Cherokee County and extends approximately halfway into Crawford County (Fig. 2). During the Pennsylvanian era, seas and swamps covered most of Kansas. This region lay peripherally to these seas, and developed into vast swampland. The vegetative detritus that settled into the bottom of these areas formed coal veins (Buchanan 2010), which were mined extensively in the Cherokee Lowlands. Spoil banks and strips of watery pits litter the landscape.

Osage cuestas: The Osage Cuestas comprise the other half of Crawford County and extend into the northwest corner of Cherokee County (Fig. 2). The cuestas form parallel ridges that slope gently westward, but more steeply eastward. These slopes alternate between shale and limestone deposits (Buchanan 2010). The highest elevations in the region occur on the Osage Cuestas at approximately 300 meters.

Land use.-Past surface mining is evident across much of Crawford and Cherokee counties. The most visible of these are the strip pits, which have evolved into small (but sometimes deep) artificial ponds or lakes, dotting much of the landscape. Coal mining, once the dominating profession here, dwindled in southeastern Kansas in the 1970's and eventually ceased after legislation was passed regulating mining companies and required cleanup (Brady 2018).

Prior to 1969, coal companies often extracted coal leaving the overburden without attempting reclamation. In 1969 the State of Kansas passed the Mined-Land Conservation and Reclamation Act, Kansas Statute Annotated 49-401 et.seq., which required coal companies to obtain permits, follow reclamation guidelines, and submit to monthly monitoring (Kansas Department of Health and Environment: Regulatory Program 2018). Thereafter, in 1977 the Surface Mining Control and Reclamation ACT (SMCRA) was passed, which established federal guidelines for coal companies (Kansas Department of Health and Environment: Abandoned Mine Land Program 2018). Companies were required to reclaim lands by refilling coal extraction areas' holes, 
and reseeding these areas. The reclamation efforts were to be monitored closely, though already abandoned pits were not reclaimed; today it is common to find both reclaimed and un-reclaimed lands throughout.

Most lands with public access in southeastern Kansas are former strip mines that are now overseen by Kansas Department of Wildlife Parks and Tourism. A total of 47 Mined Land Wildlife Areas occur throughout Crawford and Cherokee counties, totaling approximately 14,500 acres (KDWPT pamphlet). Strip pits that were either un-reclaimed or too deep to fill form small ponds and lakes, which are now stocked with fish. Many of these reclaimed areas are used currently for public recreation, with hunting and fishing being the most popular activities.

Two toxic ores, lead and zinc, were commonly extracted through underground mining activities throughout the Ozark Plateau. These ores were mined extensively from 1870-1970 in the city Galena, in Cherokee County and surrounding areas. Over 2.9 million tons of zinc and 650 thousand tons of lead were extracted from Kansas during that interval (McCauley et al. 1983). To obtain these ores, the cherty rock, common to the Ozark Plateau, was crushed and deposited in large piles known as chat piles, which still dot the landscape along the southern border of Kansas (Buchanan 2010). These piles remain heavily contaminated with lead, zinc, and cadmium and contribute significantly to the heavy metal contamination in southern Cherokee County, southwest Missouri, and northeast Oklahoma (Beyer et al. 2004; Schmitt et al. 2006; Angelo et al. 2007; Merwe et al. 2011; Manders \& Aber 2014).

Farming became the most dominant land use in southeastern Kansas after the cessation of mining. Extensive areas of the region are tilled for cropland or used for cattle grazing. The most common crops include corn, wheat, soybeans, sorghum, red clover, and hay (fescue grass, Festuca and Schedonorus).

Soils.-Silt loams are the most common soils in the Cherokee Lowlands of Cherokee and Crawford counties; the most common of which are called the Parsons silt loam. They dominate the Osage Cuestas, particularly in the northwest corner of Crawford County, covering over $90 \%$ of the soil types, representing the most common type throughout the study area. However, they are less abundant here than in the Cherokee Lowlands because the cuestas have a considerable diversity of soil types, including silt loams, silty clay loams, and silty clays. The center of Crawford County contains the Zaar silty clay, whereas the northeastern corner contains more silty clay loams (Web Soil Survey 2016).

Silt loams are the dominant soil type in the Ozark Plateau in Cherokee County. Of these, the Clarksville, a very cherty silt loam type and Nixa, a very gravelly silt loam are most prevalent. The remainder of the Ozark Plateau is composed of a variety of silt loams, whereas silty clays are typically absent (Web soil Survey 2016).To a lesser degree, loams and silty clay loams occur throughout the region (Web Soil Survey 2016).

\section{MATERIALS AND METHODS}

Collecting and processing.-Plant specimens were collected from early spring through fall in both 2014 and 2015, with a few additional collections from 2016 through 2019. Searching a given field site employed the process of "intelligent meandering," mentioned and used by some floristicians throughout North America (e.g., Legler 2010). The first author, along with occasional assistants, collected in all habitat types and physiographic regions; the second author contributed several reports and assisted in the spring and late summer of the first year and sporadically thereafter. Intensive efforts were made to collect and document all taxa occurring in the counties, except those believed to be purposefully planted. We made no attempt to determine if a taxon's presence was merely a waif.

When possible, the entire plant was collected, including its underground parts. Cuttings of woody plants of up to ca. $35 \mathrm{~cm}$ long were taken from the branch tips. All specimens were marked relative to their specific collection sites. During the first collecting season, one to several duplicates were made for most specimens, while fewer duplicates were made the following year.

Specimens were sorted by location and pressed. Most roots were rinsed and trimmed when necessary. All specimens were frozen at ca. $-20^{\circ} \mathrm{C}$ for one week before entering the Sperry Herbarium.

Each physiographic region (ecoregion) was sampled monthly, although the Cherokee Lowlands and 
Osage Cuestas were collected more frequently than the Ozark Plateau because of their large sizes. Collecting trips typically alternated between Crawford and Cherokee counties over a one or two-week period to ensure the likelihood of collecting whatever was flowering or fruiting at a given time in each county. This was subject to change depending on weather conditions.

Rare species, such as the orchid Platanthera lacera, were collected only when fifteen or more individuals were observed, then only one specimen was taken. If greater than thirty plants were observed, then two plants were taken for exchange material. If the population exceeded ca. 100 plants then three were collected. To minimize pressure on each population, most orchids were removed above the apex of the root crown.

Biases in plant collecting are well documented amongst herbarium collections (Daru et al. 2017). Since southeastern Kansas has little topographic relief, an elevation bias does not apply to our region. However, among collections in the T.M. Sperry Herbarium at Pittsburg State University (KSP following Thiers 2019), which houses among the largest number of collections from Cherokee and Crawford counties, certain collection biases are evident. These include disproportionate collecting efforts during the dominant growing seasons, collections in close proximity to major roads, spatial biases (e.g., relative distance to the nearest herbarium), and a large portion of the collections have been made by relatively few collectors. Some biases are largely unavoidable. For example, southeastern Kansas and much of the Great Plains are divided into a road grid of $1 \times 1$ miles. As such, most previous collections are within approximately 100 meters from a road, and the distance to the nearest road is not necessarily a reliable indicator of bias in this region, since few areas are less than 0.5 mile from the nearest navigable road.

Given that most land in Crawford and Cherokee counties is private, and fewer collections typically have been made on private lands, we attempted to mitigate previous spatial sampling bias by collecting on private (with landowner permission) and public lands. Private properties were surveyed mostly to maximize geographical and ecological coverage. Access to private properties occurred largely in spring of 2015, resulting in 2816 collections, or approximately $43.3 \%$ of the total. Although many landowners allowed access, not all parts of the properties could be surveyed in depth. As such, successive collecting efforts were focused on areas that appeared to have higher levels of diversity.

Other actions attempted to reduce collecting biases during this study. To reduce temporal bias, collections were made throughout the growing season, including a few in winter. Reducing individual collecting bias can be more difficult. Floristic studies of small geographic areas generally are done by a single individual, which may itself impose a certain degree of collecting bias. This level of bias can also apply to collections at smaller repositories such as that of the T.M. Sperry Herbarium, where relatively few collectors contribute the large majority of collections. One way to reduce collecting bias is to increase the number of collections made for a particular geographic area. With approximately 6450 new specimens from private and public lands, the study hopefully largely avoided any personal collection biases. Stated another way, when fully accessioned and integrated, specimens from this study increased the holdings of the Sperry Herbarium by over nine percent, and likely represent fifteen percent or more of all collections from Crawford and Cherokee counties. The collections from this study thereby should reduce whatever regional biases might have existed for Cherokee and Crawford counties from having only a few "heavy hitting" collectors from these two counties (Bebber et al. 2012).

Identification of plant specimens.-Steyermark's Flora of Missouri (Yatskievych 1999, 2006, 2013) was the primary authority used for plant identifications. With few exceptions, taxonomic names and ranks, as well as distributions at the state and county levels, followed Biota of North America's Floristic (BONAP; Kartesz 2019). BONAP not only provides the accepted nomenclature for genera, species, and infraspecific North American taxa as currently curated in the T.M. Sperry Herbarium, and follows the familial classification of the Angiosperm Phylogeny Group IV (APG IV 2016), it also indicates (where known) previously misidentified reports at the state and county levels, and strives to voucher as many reports as possible. It also is the most consistently updated source of information nationally at the state and county level, and accepts vouchered reports from reliable contributors. 
Secondary sources used for identification or confirmation included several volumes of Flora of North America, Flora of the Great Plains (GPFA 1986), and Shinners \& Mahler's Illustrated Flora of North Central Texas (Diggs et al. 1999). For several genera we used on keys in Flora of North America, including Bromus (Pavlick \& Anderton 2007), Muhlenbergia (Peterson 2003), and Amaranthus (Mosyakin \& Robertson 2003). Of particular note, the treatment in Flora of North America was used for the identification of the grass genus Dichanthelium (Freckmann \& Lelong 2003), but we report names as recognized by Kartesz (2019). Since the taxonomy of Dicanthelium is particularly difficult, with various revisions underway (e.g., Thomas 2015; Thomas pers. comm., 2018), taxonomic boundaries of some taxa will likely change.

In the relatively few cases listed below the taxonomic names or ranks were modified from Kartesz (2019) and/or from APG IV (2016), including:

Acer is maintained in Aceraceae based on the work of (Buerki et al. 2010), as opposed to its inclusion in Sapindaceae (Kartesz 2019; APG IV 2016)

Fumariaceae are placed in Papaveraceae as per APG IV (2016), instead of Fumariaceae.

Bromus commutatus and Bromus racemosus (Poaceae) are recognized as distinct following Flora of North America (Palvick \& Anderton 2007) and Yatskievych (1999), who maintain these as distinct.

Kartesz (2019) occasionally recognizes quadrinomials, as for example in Symphyotrichum lanceolatum (Asteraceae). However, the T.M. Sperry Herbarium does not recognize varieties within subspecies based on the ontological aversion of the the second author of recognizing two hierarchical levels of variation within a species, when populations within a species themselves are not expected to be hierarchical (Snow 1997). We therefore follow the taxonomy of Yatskievych (2006) in recognizing only one level of infraspecific variation.

Analysis.-All specimen data were entered into an Excel $^{\mathrm{TM}}$ database using DarwinCore data standards (Wieczorek et al. 2012) and uploaded to Symbiota (Gries et al. 2014). They are available through SEINet, and the portal of the Consortium of Northern Great Plains Herbaria (http://ngpherbaria.org/portal/profile/index. php?refurl=/portal/index.php?) and other online aggregators such as the Global Biodiversity Information Facility (GBIF 2019) in Copenhagen (http://www.gbif.org/), the only global database that attempts to collate data worldwide. All locality data can be found on those sites; barcodes are being added to specimens as they are mounted. (At the time of the revised submission, only about five percent of the specimens have been mounted.)

Geo-coordinates and elevations were taken for each collecting location using the IOS app, Elevation for Real (EFR), which is produced by homedatasheet.com INC. This app uses the USGS web service to provide GPS coordinates for locations with a horizontal confidence radius of $5 \mathrm{~m}$. Sometimes Google Earth ${ }^{\mathrm{TM}}$ was consulted at the lab if the mobile device was not working or within reach during collection. Comparing this app to Google Earth and another GPS devices used in the Sperry herbarium, EFR gave the same reading as the others mentioned for the first five decimal places.

To confirm state and county records, the currently accepted names and synonyms (Kartesz 2019) were checked against other sources. These records also were checked against online specimen data available from Kansas State University Herbarium and the McGregor Herbarium (University of Kansas). Names and potential synonyms also were checked against Gibson (1963). When unable to confirm a report for Crawford County by Gibson (1963), either at KSP or other herbaria in Kansas, we did not include that name. For state records, specimens were checked by the first and second authors. All possible state records, except for easily identified specimens, were confirmed against specimens in the Missouri-Illinois reference collection at the Missouri Botanical Garden, or at the McGregor Herbarium at University of Kansas.

Taxa that we collected that were not listed in Kartesz (2019) by any currently accepted names or synonyms for Crawford or Cherokee counties are reported as county (or state) records. It is widely understood among taxonomists that older specimens may exist in other herbaria (possibly unreported in the literature or misidentified) that represent some of the records listed. However, given that they are not reported in any of our regional or national sources, this article may be considered the first official report for many county and state records.

Reporting.-All collections were deposited in the T.M. Sperry Herbarium at Pittsburg State University. Collection numbers are primarily of the first author (Young 1-400; Pryer 401-6425), including those that were 
modified later via adding suffixes $a$ and $b$ when multiple taxa were collected for the same specimen number. This increased the total sequence of collecting numbers to 6497 , although some later were discarded due to absence of fertile material. Relevant collections of the second author to this study included over fifty of Snow $(10,736-10,776 ; 10,785-10,786 ; 10,974 ; 10,980 ; 11,138)$. Following current data in Kartesz (2019), taxa are reported as native or non-native to North America; rare, noxious, extirpated, or adventive (a native North American taxon occurring beyond its original range) and (where applicable) as State or county records. In a few cases barcode numbers of the Sperry Herbarium are indicated in parentheses of older collections in square brackets. This work strived to meet high standards for floristics research proposed by Palmer and Richardson (2012) regarding categories of information and their levels of detail.

\section{RESULTS AND DISCUSSION}

Taxonomic summary.-A total of 1420 unique taxa (species and subspecies or varieties) were documented for Crawford (1139) and Cherokee (1281) counties based on new collections and vouchered reports from Gibson (1963) and Kartesz (2019). Approximately 6450 specimens were collected for this survey, resulting in 1021 vouchered taxa. Thus, despite two complete field seasons and sporadic opportunistic collecting after the 2014-2015 field seasons, only 71.9\% of the vascular plants known to occur in the two counties were collected. This is suggestive of the time, effort, geographical coverage, and intensity of sampling required to fully document the flora of a given area, even at the county level. As another example of the effort needed to fully document a flora, species accumulation curves across five common habitat types from a floristic survey in northeastern New Mexico had not yet asymptoped after two complete field seasons that included over 9600 newly collected specimens (Schiebout et al. 2008).

Collected taxa that are absent for either Cherokee or Crawford counties in BONAP (Kartesz, 2019) as accepted names or synonyms were considered county (or state) records. The 399 known taxa for the two counties (combined) that were not collected for this project were confirmed with vouchers from Gibson's vouchers in the Sperry Herbarium or by reports from BONAP (Kartesz 2019). Of the 1021 taxa collected, 494 occurred on the Ozark Plateau, 578 on the Osage Cuestas, and 808 in the Cherokee Lowlands. Assignments to these three major ecoregions were not made for taxa that we did not collect (See Appendix for distribution of taxa by ecoregions.)

The overall totals included 148 families and 591 genera (Table 1). Angiosperms comprised $98.0 \%$ of the taxa, with 1391 specific and infraspecific taxa, including 135 families and 570 genera (Table 1). Ferns and fern allies accounted for approximately 1.8\% (11 families; 17 genera; 25 taxa) and gymnosperms approximately $0.3 \%$ of the total ( 2 families; 3 genera; 4 taxa) of the overall taxa (Table 1). Of the 1420 specific and infraspecific taxa, 1177 (ca. 82.9\%) are considered native to the North America (Kartesz 2019). Twenty-two taxa (1.5\%) in the area are native but considered adventive from elsewhere in North America. A total of 243 taxa (1.7\%) are considered "rare" in BONAP, based on information provided primarily from the Kansas Biological Survey (J. Kartesz, pers. comm.). Eight taxa presently are listed as "extirpated" in BONAP, but given the number of county and state records we discovered, reports of extirpated should not be taken as definitive.

The ten largest families collectively comprise approximately $53.1 \%$ of the flora (750 taxa), but just the five largest comprise approximately $41.5 \%$ of the diversity (586 taxa). The ten largest families typically comprise a large percentage of the taxa in similar floristic studies for most continental U.S. counties (e.g., Snow 1994; Hazlett 2004; Schiebout et al. 2008; Legler 2010). Poaceae (181 taxa; 12.7\% of the total unique taxa) was the largest family, followed by Asteraceae (180; 12.7\%), Fabaceae (106; 7.5\%), Cyperaceae (92; 6.5\%), Rosaceae (47; $3.3 \%)$, Brassicaceae (41; 2.9\%), Lamiaceae (40;2.8\%), Apiaceae (29; 2.0\%), Ranunculaceae (29; $2.0 \%)$, and Plantaginaceae $(27 ; 1.9 \%)$.

The ten largest genera comprise approximately $12.8 \%$ of the total (181 taxa), including: Carex (54 taxa), Symphyotrichum (19), Euphorbia (15), Quercus (15), Juncus (15), Solidago (14), Dichanthelium (13), Asclepias (13), Viola (12), and Desmodium (11). Many species are near the western edges of their geographical distribution. See the Appendix (Annotated Checklist) for the complete list of taxa. 
TABle 1. Taxonomic and numerical summaries. Nativity refers to North America (Kartesz 2019).

\begin{tabular}{|c|c|}
\hline \multicolumn{2}{|l|}{ List by taxonomic category, study overall } \\
\hline Families & 148 \\
\hline Genera & 591 \\
\hline Species & 1309 \\
\hline Non-nominal infraspecific taxa & 111 \\
\hline Nothotaxa & 15 \\
\hline Unique taxa & 1420 \\
\hline \multicolumn{2}{|l|}{ List by special category } \\
\hline Native taxa & 1177 \\
\hline Percent native taxa & 82.9 \\
\hline Non-native taxa & 243 \\
\hline Percent non-native taxa & 17.1 \\
\hline KS noxious taxa & 53 \\
\hline State records & 44 \\
\hline Crawford County records & 152 \\
\hline Cherokee County records & 81 \\
\hline \multicolumn{2}{|l|}{ List of unique taxa by major plant group } \\
\hline Ferns and Fern Ally families & 11 \\
\hline Ferns and Fern Ally genera & 17 \\
\hline Ferns and Fern Ally taxa & 25 \\
\hline Ferns and Fern Ally native taxa & 25 \\
\hline Ferns and Fern Ally non-native taxa & 0 \\
\hline Gymnosperm familes & 2 \\
\hline Gymnosperm genera & 3 \\
\hline Gymnosperm taxa & 4 \\
\hline Gymnosperm taxa native & 4 \\
\hline Gymnosperm taxa non-native & 0 \\
\hline Angiosperm families & 135 \\
\hline Angiosperm genera & 568 \\
\hline Angiosperm taxa & 1382 \\
\hline Angiosperm taxa native & 1130 \\
\hline Angiosperm taxa non-native & 241 \\
\hline
\end{tabular}

Non-native and invasive taxa.- Non-natives were represented by 243 taxa ( $17.1 \%$ of the unique taxa), a figure considerably higher than some areas (e.g., 9.3\% [Schiebout et al. 2008] and 10.5\% [Larson et al. 2014]). Of the 1420 total taxa, $54(3.8 \%)$ are considered noxious by various state agencies. The noxious taxa were spread across 14 families and 30 genera, including 23 considered native and 31 non-native. Convolvulaceae had the highest number with 11 noxious taxa, due primarily to the genus Cuscuta. The Cherokee Lowlands had the highest number of noxious plants with 33 taxa. Twenty-five noxious taxa were recorded for the Osage Cuestas and 15 for the Ozark Plateau.

The significant percentage of non-native taxa is unsurprising given that both counties have been surfacemined or farmed heavily. The tallgrass prairies historically native to southeastern Kansas now are restricted to small remnant patches, such as the O'Malley Prairies owned by Pittsburg State University, and some privately owned parcels. Roadsides in southeastern Kansas sometimes retain remnant prairie patches. Gibson (1963) correctly predicted that a majority of the remaining prairie parcels he had surveyed would disappear. Much of his collecting was from the northwest corner of Crawford County, but most of that area now has been tilled, with few existing native prairie remaining. According to several local landowners (pers. comm. to SYP), some remnants are in danger of being converted to cropland.

Many of the prairie remnants in southeastern Kansas are rarely if ever burned. The lack of fire may promote the invasive non-native species, including fescue grasses (Schedonorus and Festuca) and ox-eye daisy (Leucanthemum). Longer absences of fire can give way to woody species such as the native cedar (Juniperus virginiana) and green ash (Fraxinus americana). Given that some landowners take great pride in maintaining native prairies, there is room for hope that some will remain in perpetuity. 
Callery Pear (Pyrus calleryana), also known as Bradford Pear, has naturalized in both counties and is documented in both for the first time. It is native to China and was imported to the U.S.A. in 1917 to help combat fire blight in the pear tree, Pyrus communis (Culley \& Hardiman 2007). It has become a common landscape tree because of its fast growth, pyramidal growth habit, and abundant white flowers in early spring. However, it is now considered an invasive species occurring across the eastern half of the U.S.A. and reported westward into California and Utah. The Missouri Department of Conservation, Kansas Department of Wildlife Parks and Tourism, Kansas Wildlife Federation, the New York State Urban Forestry Department and others are requesting that people stop planting this tree, despite its attributes. Efforts are underway in several states, including Kansas to remove the species because of its invasive tendencies. Another widely planted and now weedy tree is Elaeagnus umbellata, which is spreading rapidly in some areas in the Cherokee Lowlands.

Rare species.-Of the approximately 500 rare taxa tracked for Kansas (Kartesz 2019), 243 (17.1\% of the total unique taxa) have been reported in Crawford or Cherokee counties. Corroborating current data suggesting they are rare, 127 (52.3\%) of the 243 rara taxa were not found during the present study. Cyperaceae included the most rare taxa (36). The most rare taxa at the generic level was Carex (21 taxa, of which 14 were collected during this study). Rare taxa collected during this study included 55 from the Ozark Plateau, 50 in the Cherokee Lowlands, and 27 in the Osage Cuestas (with many occurring in more than one of these ecoregions). Limestone bedrocks in the Ozark Plateau comprise only a small part of Cherokee County, but plant species often have high fidelity on these calcareous substrates (Yatskievych 1999). Cherokee County is the only one in Kansas with this bedrock, which likely explains why it also has the most rare taxa among counties in the state (Kartesz 2019). The Cherokee Lowlands, which comprise the remainder of Cherokee County, have a relatively small total coverage in Kansas.

Species of special concern and rare taxa.-Mead's milkweed (Asclepias meadii; Apocynaceae) and the western prairie fringed orchid (Platanthera praeclara; Orchidaceae) are federally listed as Threatened. Their historical ranges extended into southeastern Kansas. Asclepias meadii has been reported for most of the eastern tier counties of Kansas, including Crawford County (USFWS 2003; Kartesz 2019), and Kindscher et al. (2009) indicated that eastern Kansas is the core of its range. Platanthera praeclara has been reported in several of the northeastern counties in Kansas and also Crawford County. Despite considerable effort to relocate these species, no populations were found in Cherokee or Crawford counties. The state of Kansas does not report plants as becoming species of concern, threatened, or endangered. However, it does list taxa it considers rare. Of the 24 taxa listed as rare for Kansas (Kartesz 2019), 18 were collected as county records for Crawford County and 6 for Cherokee County.

Noxious taxa.-Designation of a taxon as noxious is made individually by each state, and may apply to native or non-native taxa. A designation of noxious in Kansas applies to six of the county records and one state record.

New records for Kansas.-Although many represent relatively short-distance range extensions or fillins between neighboring counties, a higher number of state and county records were discovered during this study than expected. Forty-four taxa new to Kansas are reported here, representing 26 families and 42 genera (Table 2). The current number of vouchered, unique taxa in Kansas is 2479 (Kartesz 2019). Interestingly, only approximately one quarter of the state records $(12 ; 27.3 \%)$ were non-native taxa, and of these only three are adventive from ornamental plantings (Leucojum aestivum subsp. aestivum, Narcissus poeticus, and Syringa vulgaris). One, Allium vineale subsp. compactum, is considered noxious in surrounding states, and all taxa of Allium are considered noxious in Kansas.

A total of 233 county records are reported for combined totals of Crawford (152) and Cherokee (81) counties, representing 193 unique taxa. (Country records herein exclude designations of taxa as Kansas state records.) Of the 193 unique taxa representing new county records, 55 are non-native and 138 are native. Somewhat unexpectedly, the number of county records for taxa native to North America is $257 \%$ (= $139 / 54$ [100\%]) higher than the non-native taxa. These ratios for southeast Kansas indicate that new county and state records represent substantially more than uncollected non-native (and often weedy) taxa. 
TABLE 2. First published state records for Kansas, including counties in which they were collected and nativity status; see Appendix for voucher specimens and other discussions).

\begin{tabular}{|c|c|c|c|}
\hline Family & Species Name & County collected & Nativity \\
\hline Alliaceae & Allium vineale subsp. compactum & Cherokee; Crawford & Non-native \\
\hline Altingiaceae & Liquidambar styraciflua & Cherokee; Crawford & Native \\
\hline \multirow[t]{2}{*}{ Amaryllidaceae } & Leucojum aestivum subsp. aestivum & Crawford & Non-native \\
\hline & Narcissus poeticus & Crawford & Non-native \\
\hline Aquifoliaceae & Ilex opaca var. opaca & Crawford & Native \\
\hline Araliaceae & Hederahelix & Crawford & Non-native \\
\hline \multirow[t]{3}{*}{ Asteraceae } & Euthamia leptocephala & Crawford & Native \\
\hline & Fleischmannia incarnata & Cherokee & Native \\
\hline & Solidago arguta var. caroliniana & Cherokee & Native \\
\hline Bignoniaceae & Catalpa ovata & Crawford & Non-native \\
\hline Blechnaceae & Woodwardia areolata & Crawford & Native \\
\hline Boraginaceae & Myosotis macrosperma & Crawford & Native \\
\hline \multirow[t]{2}{*}{ Brassicaceae } & Cardamine hirsuta & Cherokee; Crawford & Non-native \\
\hline & Erysimum capitatum & Crawford & Native \\
\hline \multirow[t]{2}{*}{ Caryophyllaceae } & Arenaria serpyllifolia var. tenuior & Cherokee & Non-native \\
\hline & Stellaria neglecta & Cherokee; Crawford & Non-native \\
\hline Convolvulaceae & Cuscuta compacta var. compacta & Crawford & Native \\
\hline Cyperaceae & Carex amphibola & Crawford & Native \\
\hline \multirow[t]{3}{*}{ Fabaceae } & Desmodium viridiflorum & Cherokee & Native \\
\hline & Lathyrus venosus & Cherokee & Native \\
\hline & Lespedeza frutescens & Cherokee & Native \\
\hline \multirow[t]{2}{*}{ Fagaceae } & Quercus falcata & Cherokee & Native \\
\hline & Quercus michauxii & Cherokee & Native \\
\hline Hypericaceae & Hypericum gymnanthum & Crawford & Native \\
\hline Juncaceae & Juncus secundus & Crawford & Native \\
\hline \multirow[t]{2}{*}{ Lamiaceae } & Callicarpa americana & Crawford & Native \\
\hline & Salvia farinacea & Crawford & Native \\
\hline Oleaceae & Syringa vulgaris & Cherokee & Non-native \\
\hline Pinaceae & Pinus taeda & Cherokee & Native \\
\hline \multirow[t]{8}{*}{ Poaceae } & Bromus sterilis & Cherokee; Crawford & Non-native \\
\hline & Diarrhena americana & Crawford & Native \\
\hline & Elymus riparius & Cherokee; Crawford & Native \\
\hline & Eragrostis pilosa var. pilosa & $\mathrm{CR}$ & Native \\
\hline & Microstegium vimineum & Cherokee & Non-native \\
\hline & Paspalum dilatatum & Cherokee & Native \\
\hline & Sorghum bicolor subsp. drummondii & Cherokee & Non-native \\
\hline & Urochloa platyphylla & Cherokee & Native \\
\hline Ranunculaceae & Ficaria verna subsp. calthifolia & Cherokee & Non-native \\
\hline Rosaceae & Geum virginianum & Cherokee; Crawford & Native \\
\hline Salicaceae & Populus deltoides subsp. deltoides & Crawford & Native \\
\hline Smilacaceae & Smilax glauca & Crawford & Native \\
\hline \multirow[t]{2}{*}{ Solanaceae } & Solanum americanum & Crawford & Native \\
\hline & Solanum sarrachoides & Cherokee; Crawford & Non-native \\
\hline Violaceae & Viola affinis & Cherokee & Native \\
\hline
\end{tabular}

Some taxa collected previously in Kansas, such as Solanum sarrachoides, Eragrostis pilosa var. pilosa, and Allium vineale subsp. compactum, to the best of our knowledge had not been published (Kartesz 2019). A few to many specimens of each were found during this study in T.M. Sperry Herbarium (Pittsburg State University) and R.L. McGregor Herbarium (University of Kansas). In the case of Eragrostis and Allium, our infraspecific designations are not recognized at R.L. McGregor Herbarium, although these taxa were observed among specimens there (SYP, pers. obsv., March 2018). To re-emphasize, these taxa have been present in Kansas for some time, and their reports here reflect a discrepancy of current taxonomic concepts.

Range expansions.-Several surprising range expansions were noted. For example, Stellaria neglecta (Caryophyllaceae) was known previously from Newton County, Arkansas (ca. $200 \mathrm{~km}$ ). Although the first 
author confirmed this taxon in Kansas early in 2014, its regional occurrence was noted by Snow et al. (2017) in Ottawa County, Oklahoma. Interestingly, Stellaria neglecta had been recorded previously only in eleven counties across the U.S.A. (Kartesz 2019). Perhaps because of its non-native status, it had been reported infrequently, and in fact most floras do not include it in keys and descriptions. It closely resembles $S$. media, the common chickweed that occurs across the U.S.A., differing by its conical tubercles on the mature seeds, in contrast to the hemispherical tubercles of S. media. Collections of S. neglecta documented in Crawford and Cherokee counties comprise almost half of the collections made of Stellaria for this study. It likely is common in surrounding counties as well.

A second surprising range expansion was Ficaria verna subsp. calthifolia (Ranunculaceae), a non-native taxon with the closest reported occurrence being in St. Louis County, Missouri, ca. $400 \mathrm{~km}$ to the east. The specimens occurred in low-lying and moist areas, which are abundant in the Cherokee Lowlands. Most documented records for this species, including all of the recognized subspecies, occur along the East coast, with a sparse occurrence into the Midwest. Nesom (2008) reported it naturalizing in Texas and later noted that the population continues to grow locally (Nesom 2015). It was first introduced to the U.S.A. in the $19^{\text {th }}$ century as a garden ornamental (Post et al. 2009). The specimens collected in Kansas were found growing abundantly on a private property in Cherokee County. The owner, who is now retired, recalls it as being common on his property throughout most of his lifetime, but he did not plant it (anonymous pers. comm. to SYP, 2014). Its origin in Kansas is unknown, and on the property it is abundant in areas that are neither landscaped nor mowed. Post et al. (2009) also indicated that Ficaria verna and its several subspecies are likely to naturalize where introduced in New England. They expected its existence in Kansas in low irrigated areas or wetlands, though unirrigated areas of Kansas are usually too arid for this species to persist. Southeastern Kansas has more average annual precipitation than the rest of the state, thus its continued naturalization within the region is likely.

A comparison of the specimens of Ficaria verna subsp. calthifolia (Ranunculaceae) was made with materials from Missouri-Illinois Reference collection of Missouri Botanical Garden. Our materials keyed out well using Flora Europaea (Tutin 1964), except that the achenes were mostly glabrous. The specimen keys out well using Post et al. (2009), as the leaves are crowded at the base, not on elongated stems, and are not $4 \times 4 \mathrm{~cm}$ or less.

A specimen of Catalpa ovata (Bignoniaceae) was collected from a pasture in Crawford County. The specimen was a small tree not planted by the owner, but subsequently cut during haying season. A dense patch of suckering shoots was observed the following year. No additional eradication measures were planned (anonymous pers. comm. to SYP, 2014). Catalpa ovata has been recorded for 12 states, mostly northern New England states, to West Virginia, Ohio, extending into Pennsylvania, Minnesota, and Wisconsin. The closest records to Kansas are from Boone (ca. $300 \mathrm{~km}$ ) and Crawford (ca. $325 \mathrm{~km}$ ) counties in Missouri. It was reported in one county of Nebraska, but eradicated from the state (Kartesz 2019). The genus Catalpa was planted widely, with significant plantings also of Maclura pomifera, Castanea (chestnut), Juglans nigra (black walnut), and Ailanthus glandulosa (ailanthus), in the long-defunct St. Louis-San Francisco ("Frisco") Tree Farm in Farlington, about $18 \mathrm{~km}$ to the north-northwest from where C. ovata was collected. However, the last tree harvests occurred in 1918 (Nelson 1952). It is possible that farm was a source of seed to the area where it was collected for this study.

Our records of Allium vineale subsp. compactum (Alliaceae) are not surprising, but noteworthy. It is considered noxious in surrounding states. This taxon has been reported only in four states and eight counties nationwide (Kartesz 2019), though some of these reports may be due to taxonomic confusion. Allium vineale subsp. vineale is widespread throughout the eastern half of the U.S.A., and some to possibly many of those collections may be subspecies compactum; moreover, some herbaria may not recognize this taxon at the infraspecific level. For instance, both expressions were observed in the T.M. Sperry Herbarium and R.L. McGregor Herbarium, not yet differentiated.

Leucojum aestivum subsp. aestivum (Amaryllidaceae) was a surprising find and a significant range expansion, with the closest record being in Boone County, Missouri (ca. $300 \mathrm{~km})$. It appears to be rather common in some of the southern states, including Louisiana and Alabama; with spotty distributions along the east coast and with some occurrences in California and one from Oregon. The specimens were growing abundantly 
along a small creek bed in Schlanger Park in Pittsburg, where 100+ plants were observed. Since the population was hidden in the trees along a creek and otherwise not planted, it is here considered naturalized.

Several plants of Salvia farinacea (Lamiaceae) were found in the State of Kansas Mined Land Wildlife Areas, where they likely had re-established naturally, given that these areas typically were not reclaimed after the cessation of surface mining activities (Bailey \& Hooey 2017). The closest record is from Delaware County, Oklahoma (ca. $100 \mathrm{~km}$ ). This taxon has a limited distribution across the U.S.A., persisting mostly in southern arid areas of Texas and New Mexico, with records in seven counties in Oklahoma, three in Louisiana, and two in Florida. It has been reported and labeled as adventive in Ohio and Connecticut. One specimen was found for Kansas in the R.L. McGregor Herbarium (Holland 5857, Neosho County, KS September 4, 1987), though it was listed as "under cultivation." This is the first report of this taxon occurring outside of cultivation for the state.

Callicarpa americana (Lamiaceae) was reported previously in four counties of Missouri and listed as rare for that state in BONAP (Kartesz 2019). It is common in the southeastern U.S.A, with Kansas being at northern terminus of its range (Kartesz 2019). It has also been reported as occurring in Virginia and Maryland, with its closest records from Taney County, Missouri (ca. 170 km), Washington County, Oklahoma (ca. 150 km), and Washington County, Arkansas (ca. 160 km).

Quercus michauxii (Fagaceae) is distributed throughout much of the southeastern U.S.A, with its closest record in Carroll County, Arkansas (ca. 150 km). Kansas represents the northwestern extent of its known range. The farthest west it had been reported is Canadian County, Oklahoma. This specimen comes from Schermerhorn Park, a nature park in Cherokee county. This park preserves the natural flora of the Ozark Plateau and according to the caretakers they avoid plantings (J. Rader, park manager, pers. comm. to S. Pryer, 2014). Though some work to make trails more accessible and remove invasive species has been done, to our knowledge no tree plantings have occurred.

Extirpated Taxa.—Eight taxa are considered extirpated from Kansas (Kartesz 2019). However, of these, the first author collected four taxa and Gibson (1963) reported two more. Species collected for this study previously considered extirpated in Kansas are included in all the tallies and the Annotated Checklist.

Exclusions.-A few taxa excluded from the tallies are considered remnants from previous plantings they are included in the Annotated Checklist but excluded from numerical tallies. We note here their presence for the benefit of future workers: Castanea mollissima, Crocus vernus, Chaenomeles speciosa, and Forsythia suspensa. Gibson (1963) earlier reported Broussonetia papyrifer, Cornus foemina, Viburnum rafinesquianum, and Ziziphus jujuba.

Identification notes.-Specimens of Plantago lanceolata (Plantaginaceae) did not always key easily in Yatskievych (2013). However, they keyed well in Kaul et al. (2011). Many specimens of P. lanceolata have bracts that are conspicuously hairy on the midrib, not just the margins. Since Yatskievych (2013) uses this to separate the species, P. lanceolata typically fell outside of the key.

Two specimens of Cyperus lupulinus (Cyperaceae; Pryer 5104 and 5666) show a similar discrepancy when keying to subspecies. Both specimens have only three florets, which would indicate subsp. macilentus, but their scales are $4.0 \mathrm{~mm}$ and $3.3 \mathrm{~mm}$ (respectively), which would indicate subsp. lupulinus. For this reason, subspecific designation was not studied.

Kartesz (2019) indicates Coreopsis grandiflora var. harveyana (Asteraceae) as being common in Kansas. However, given that none of the primary identification sources (Steyermark's Flora of Missouri, Flora of North America, Flora of the Great Plains) provide identification information for varieties, we do not include varietal designations.

As noted in the Methods, for Prunus (Ranunculaceae) we follow the treatment in Flora of North America, which reports Prunus rivularis (Rosaceae), but not Prunus munsoniana. If both were recognized taxonomically, then both would occur here.

A specimen of Juncus brachycarpus (Pryer 1830) (Juncaceae) appears to have vegetative bulblets growing from the inflorescence. If so, this might be the first reported case for Juncus (Grant 1981). However, the bulblets may be an effect of an endophytic fungus. Fungal endophytes are found more commonly on grasses, though 
one case was reported for Juncus effusus in New Hampshire (Kilpatrick et al. 1961). Clay (1990) suggested that this was probably a case of host range expansion, given that there has only been one other case reported in rushes. Another possibility, and maybe a more likely one, is that the appearance of apomixis is evidence of a gall caused by a psyllid (Patch 1916; Hodkinson 1984; Wier 1986). These galls at maturity have a much different appearance than that present on this specimen, however it may just be in the early stages of growth.

Finally, several specimens of Desmodium (Fabaceae) could not be identified confidently to species, given that their flowers were absent, in bud only, or had flowers much larger than any listed in the identification sources (Pryer numbers 1742, 2202, 2412, 2053, 3050, and 3197). These could be of interest to revisionary taxonomists and may represent one or more range expansions.

\section{CONCLUSIONS}

This work represents the first in-depth floristic survey for Cherokee County and the first extensive survey published for Crawford County in 57 years.

Prior to this survey, a total of 1300 unique vascular plant taxa (species, nothospecies, subspecies, and varieties) had been reported for (combined) Crawford and Cherokee counties in southeastern Kansas (1169 for Cherokee Co. and 1007 for Crawford Co) (Gibson 1963; Kartesz 2019). Here we report a total of 1420 taxa for the combined counties (1281 for Cherokee Co. and 1139 for Crawford Co.), representing known increase in vascular plant taxa of $9.2 \%$ overall, including $9.6 \%$ for Cherokee County, and $13.1 \%$ for Crawford County. The 44 state and 233 county records documented for Crawford and Cherokee counties were substantially higher than expected.

Plant collecting in most areas of North America has declined since the 1970s (Prather et al. 2004 a,b), as have field studies in general (Ríos-Saldaña et al. 2018), despite the fact that specimens are being used more extensively now than ever before (e.g., Pyke \& Ehrlich 2010; Lavoie 2013). Since publication of the Flora of the Great Plains (GPFA 1986), relatively few large-scale floristic studies have occurred in the Midwest, including none in southeastern Kansas. Although we suspect there is a persisting notion (even among some botanists) that plant distributions in North America are well documented, our study and those of others strongly suggest otherwise at county and even state levels (Hartman \& Nelson, 1998; Ertter 2000; Schiebout et al. 2009; Legler 2010; Charboneau 2013; Larson et al. 2014; Martine \& Ward 2013; Snow et al. 2017; Gillespie et al. 2017).

The taxonomic and distributional data from floristic surveys provide valuable information to land managers and policy makers, and such data can be used in numerous ways for ecological studies, particularly now that data increasingly are available online (Funk 2004; Chapman 2005; Bebber et al. 2010, 2012; Pyke \& Ehrlich 2010; Culley 2013; Lavoie 2013; Daru et al. 2017; Queenborough 2017; James et al. 2018).

Future Work.-Additional floristic and vegetative studies that focus on the Mined Land Wildlife Areas in southeastern Kansas would be useful. In particular, floristic quality assessments, analyses of species richness, and the production of site-specific lists of taxa on the numerous mined areas would be useful to managers of these areas. 
APPENDIX

Annotated Checklist of the Vascular Plants of Crawford and Cherokee counties, Kansas.

The Checklist is organized by Pteridophytes and Lycophytes (Ferns and Fern-Allies), Gymnosperms, and Angiosperms. All families and taxa within each family are arranged alphabetically. Common names follow Kartesz (2017), as do the rare, noxious, or adventive designations. These designations, as well as all state and county records are bolded. Voucher numbers for both state and county records are also provided. Commentary is provided for certain taxa, including distribution summaries for state records and rare plants. For the purpose of this study, if a taxon was reported in $\mathbf{0 - 2 0}$ counties in the combined surrounding states (Missouri, Oklahoma, and Arkansas), it was considered rare for the area, but if reported for 20 or more counties, it was considered common for the area. The widely used phrase "4-state region" refers to the general area including southeast Kansas, northwest Oklahoma, southwest Missouri, and northwest Arkansas.

Gibson (1963) did not recognize infraspecific expressions for many species complexes. For these, when two or more expressions were recognized, an additional name was entered into the list, indicating that although he collected the species, he did not differentiate expressions. In these cases, only taxa with varieties or subspecies are included within the tally results.

\section{LEGEND}

!- Collected by Pryer

G - Collected by Gibson

$\mathbf{K}$ - Reported by Kartesz
CH - Cherokee County
OC - collected in the Osage Cuestas
OP - collected in the Ozark Plateau
CL-collected in the Cherokee Lowlands CRW - Crawford County

\section{Example:}

Taxon name Authors - Common Name. Counties collected [Collectors]. Nativity. Notes including rare, noxious, extirpated, or adventive. Physiographic region. Record (vouchers). Additional commentary.

\section{FERNS AND FERN ALLIES}

\section{ASPLENIACEAE (Spleenwort Family)}

Asplenium platyneuron (L.) B.S.P. - Ebony Spleenwort. CH [!, K], CRW $[!, \mathrm{K}, \mathrm{G}]$. Native. OP, OC, CL.

Asplenium resiliens Kunze - Black-Stem Spleenwort. $\mathrm{CH}[\mathrm{K}]$ Native. Asplenium rhizophyllum L. - Walking Fern. CH [!, K], CRW [G]. OP.

\section{BLECHNACEAE (Chain Fern Family)}

Woodwardia areolata (L.) T. Moore - Netted Chain Fern. CRW [!]. Native. CL. State Record (Pryer 1462). Collected on a mined land in Crawford County. Abundant with hundreds of fronds present. This taxon is considered rare in the state of Missouri, with the closest records in Barton and Lawrence counties. It is common in the southeastern and eastern potion of the United States, including Arkansas where it has been reported in all but two counties.

CYSTOPTERIDACEAE (Bladder Fern Family)

Cystopteris protrusa (Weath.) Blasdell - Lowland Bladder Fern. $\mathrm{CH}$ $[!, \mathrm{K}], \mathrm{CRW}[!, \mathrm{K}]$. Native. OP, CL.

Cystopteris tennesseensis Shaver - Tennessee Bladder Fern. CH [!, K], CRW [!, K]. Native. OP, OC, CL.

\section{DENNSTAEDTIACEAE (Bracken Fern Family)}

Pteridium aquilinum var. pseudocaudatum (Clute) Heller - $\mathrm{CH}$ [K]. Native. Extirpated. The most recent specimen located was made on August 21, 1949 (R.L. McGregor 3863) and is located at McGregor Herbarium, University of Kansas.

DRYOPTERIDACEAE (Wood Fern Family)

Dryopteris marginalis (L.) A. Gray - Marginal Wood Fern. CH [K]. Native.

Polystichum acrostichoides (Michx.) Schott - Christmas Fern. CH [!, K], CRW [K]. Native. Rare. OP. Reported from nine counties in Kansas. Common throughout the eastern United States and surrounding states.

ONOCLEACEAE (Sensitive Fern Family)

Onoclea sensibilis L. - Sensitive Fern. CRW [G]. Native.

OPHIOGLOSSACEAE (Adder's-Tongue Family)

Botrypus virginianus (L.) Holub - Rattlesnake Fern. CH [!, K], CRW [!, $\mathrm{K}, \mathrm{G}]$. Native. OP, OC, CL.

Ophioglossum engelmannii Prantl - Limestone Adder's-Tongue. $\mathrm{CH}$ [K], CRW [K]. Native.
Sceptridium dissectum (Spreng.) Lyon - Cut-Leaf Grape Fern. CH [K], CRW [!, K]. Native. CL.

POLYPODIACEAE (Polypody Fern Family)

Pleopeltis polypodioides (L.) Andrews \& Windham var. michauxiana (Weatherby) Andrews \& Windham - Resurrection Fern. $\mathrm{CH}$ [!]. Native. Rare. OP. Cherokee County Record (Pryer 5097, Pryer 5648).

PTERIDACEAE (Maidenhair Fern Family)

Adiantum pedatum L. - Northern Maidenhair. $\mathrm{CH}[\mathrm{K}]$, CRW [K,]. Native.

Argyrochosma dealbata (Pursh) Windham - Powdery False Cloak Fern. $\mathrm{CH}[\mathrm{K}], \mathrm{CRW}[\mathrm{K}, \mathrm{G}]$. Native.

Myriopteris alabamensis (Buckl.) Grusz \& Windham - Alabama Lip Fern. $\mathrm{CH}$ [K]. Native.

Myriopteris gracilis Fée. - Slender Lip Fern. CH [K]. Native.

Myriopteris lanosa (Michx.) Grusz \& Windham. - Hairy Lip Fern. CH $[!, \mathrm{K}]$. Native. Rare. OP. Reported from seven counties in Kansas. Common in surrounding states.

Myriopteris tomentosa (Link) Fée - Woolly Lip Fern. Native. Extirpated.

Pellaea atropurpurea (L.) Link - Purple-Stem Cliffbrake. CH [!, K], CRW [!, K]. Native. OP, OC.

Pellaea glabella Mett. ex Kuhn subsp. glabella - Smooth Cliffbrake. $\mathrm{CH}[\mathrm{K}], \mathrm{CRW}[\mathrm{K}, \mathrm{G}]$. Native. Gibson did not recognize subspecies of this taxon.

THELYPTERIDACEAE (Maiden Fern Family)

Phegopteris hexagonoptera (Michx.) Fée - Broad Beech Fern. $\mathrm{CH}$ [K]. Native. Extirpated.

WOODSIACEAE (Cliff Fern Family)

Woodsia obtusa (Spreng.) Torr. - Blunt-Lobe Cliff Fern. CRW [G]. Gibson did not identify his specimens to subspecies.

Woodsia obtusa (Spreng.) Torr. subsp. obtusa-Blunt-Lobe Cliff Fern. $\mathrm{CH}[!, \mathrm{K}], \mathrm{CRW}[!, \mathrm{K}]$. Native. OP, CL.

Woodsia obtusa (Spreng.) Torr. subsp. occidentalisWindham - BluntLobe Cliff Fern. CH [K], CRW [!, K]. Native. OC.

\section{GYMNOSPERMS}

CUPRESSACEAE (Cypress Family)

Juniperus virginiana L. var. virginiana - Eastern red-cedar. $\mathrm{CH}[!, \mathrm{K}]$, CRW [!, K, G]. Native. OC, CL. 
Taxodium distichum (L.) L.C. Rich. var. distichum - Southern BaldCypress. CRW [!, K]. Native. Adventive. CL. Gibson originally listed Taxodium distichum, however his specimens were young Juniperus virginiana var. virginiana.

PINACEAE (Pine Family)

Pinus banksiana Lamb. - Jack Pine. CRW [!, K]. Most likely long-ago introduced to the PSU's Natural History Reserve, where it has been collected previously, and possibly adventive. CL.

Pinus taeda L. - Loblolly Pine. CH [!]. CL. Adventive. State Record (Pryer 3755 [KSP023158], Pryer 6427 [KSP024294]). The specimens were taken from an older large tree in the middle of Mined Land Wildlife Area \#27, one of the largest such areas in southeastern Kansas, and an nearby younger tree. The two large and one small trees in the immediate vicinity have no indication, given their locality, of being intentional plantings. A separate area nearby indicated several other trees of various age classes, including some areas that are mostly only accessible by boat. No obvious human establishments are nearby. This species occurs mostly in the southeastern United States, with the nearest current native range being northcentral Arkansas (e.g., Pope County). Kartesz (2019) reports it as beyond its native range in six counties in Missouri, with the nearest being Polk Co.

\section{ANGIOSPERMS}

ACANTHACEAE (Acanthus Family)

Dicliptera brachiata (Pursh) Spreng. - Branched Foldwing. CH [!, K], CRW [!, K, G]. Native. OP, OC, CL.

Justicia americana (L.) Vahl - American Water-Willow. CH [!, K], CRW [!, K, G]. Native. OP, OC.

Ruellia humilis Nutt. - Fringe-Leaf Wild Petunia. CH [!, K], CRW [!, K, G]. Native. OP, OC, CL.

Ruellia strepens L. - Limestone Wild Petunia. CH [!, K], CRW [!, K, G]. Native. OP, OC, CL.

\section{ACERACEAE (Maple Family)}

Acer floridanum (Chapman) Pax - Florida Maple. CH [K]. Native.

Acernegundo L. var. negundo - Ash-Leaf Maple. CH [!, K], CRW [!, K, G]. Native. OP, OC, CL.

Acer saccharinum L. - Silver Maple. CH [!, K], CRW [!, K, G]. Native. $\mathrm{OP}, \mathrm{CL}$.

Acer saccharum Marshall var. saccharum - Sugar Maple. CH [!, K], CRW [!, K, G]. Native. OP, OC, CL.

ACORACEAE (Calamus Family)

Acorus calamus L. - Single-Vein Sweetflag. CH [K], CRW [K, G]. Non-native.

ADOXACEAE (Muskroot Family)

Sambucus nigra L. subsp. canadensis (L.) R. Bolli - Black Elder. CH [!, $\mathrm{K}]$, CRW [!, K, G]. Native. OC, CL.

Viburnum prunifolium L. - Smooth Blackhaw. CH [!, K], CRW [!, K, G]. Native. OP, CL.

Viburnum rufidulum Raf. - Rusty Blackhaw. CH [!, K], CRW [!, K, G]. Native. OP, OC, CL.

AGAVACEAE (Century-Plant Family)

Camassia angusta (Engelm. \& A. Gray) Blank. - Prairie Camas. $\mathrm{CH}$ [K], CRW [!, K]. Native. OC, CL.

Camassia scilloides (Raf.) Cory - Atlantic Camas. CH [K], CRW [!, K, G]. Native. OC. CL.

Yucca arkansana Trel. - Arkansas Yucca. CH [K], CRW [K]. Native. Rare. Yucca flaccida Haw. - Weak-LeafYucca. CH [!, K], CRW [!, K, G]. Native. Adventive. OP, CL.

Yucca glauca Nutt. - Soapweed Yucca. CH [!]. Native. CL. Cherokee County Record (Pryer 4959).
ALISMATACEAE (Water-Plantain Family)

Alisma subcordatum Raf. - American Water-Plantain. CH [!, K], CRW [K, G]. Native. CL.

Alisma triviale Pursh - Northern Water-Plantain. CR [!]. Native. CL. Crawford County Record (Pryer 2118).

Echinodorus berteroi (Spreng.) Fassett - Upright Burrhead. CH [!], CR [K]. Native. CL. Cherokee County Record (Pryer 5566).

Echinodorus cordifolius (L.) Griseb. - Creeping Burrhead. CH [!, K]. Native. OP, CL.

Sagittaria ambigua J.G. Sm. - Kansas Arrowhead. CH [K]. CRW $[K, G]$. Native. Rare. Reported in eleven counties in Kansas. Considered rare across its distribution and reported in only five states: Kansas, Oklahoma, Missouri, Illinois, and Indiana. Most common in surrounding areas.

Sagittaria brevirostra Mack. \& Bush - Short-Beak Arrowhead. CH [!, K], CRW [!, K, G]. Native. OP, CL.

Sagittaria calycina Engelm. - Hooded Arrowhead. CH [K], CR [K, G]. Native.

Sagittaria graminea Michx. - Grass-Leaf Arrowhead. CH [K].

Sagittaria latifolia Willd. - Duck-Potato. CH [K, G]. Native.

ALLIACEAE (Onion Family)

Allium canadense L. var. canadense - Meadow Garlic. CH [!, K], CRW $[!$, K]. Native Noxious. CL.

Allium canadense L. var. lavendulare (Bates) Ownbey \& Aase Meadow Garlic. CH [!, K], CRW [!, K]. Native Noxious. CL.

Allium canadense L. var. mobilense (Regel) Ownbey-Meadow Garlic. $\mathrm{CH}[!, \mathrm{K}], \mathrm{CRW}[!, \mathrm{K}, \mathrm{G}]$. Native. Noxious. OC, CL.

Allium sativum L. - Cultivated Garlic. CH [!, K], CRW [!, K, G]. Nonnative. Noxious. CL.

Allium stellatum Fraser ex Ker-Gawl. - Autumn Onion. CH [!], CRW [!, K]. Native. Noxious. OP, OC, CL. Cherokee County Record (Pryer 1205, Pryer 1623).

Allium vineale L. subsp. compactum (Thuill.) Coss. \& Germ. - Crow Garlic. CH [!], CRW [!]. Non-native. State record (Pryer 1082). $\mathrm{OP}, \mathrm{CL}$.

Allium vineale L. subsp. vineale - Crow Garlic. CH [!, K], CR [K]. Nonnative. Noxious. OC.

Nothoscordum bivalve (L.) Britt. - Crowpoison. CH [!, K], CRW [!, K, G]. Native. OP, OC, CL.

ALTINGIACEAE (Sweet-Gum Family)

Liquidambar styraciflua L. - Sweet-Gum. CH [!], CRW [!]. Native. CL. State Record Cherokee County Vouchers (Pryer 3744, Pryer 3863) Crawford County Voucher (Pryer 6117). Naturalizing and prevalent on mined land areas in Cherokee County and occasionally in Crawford County. Abundant in southeastern United States, including the 4-state region. Reported in all but four counties in Arkansas and scattered in Oklahoma and Missouri, the closest record being Benton County, Arkansas. One specimen was located in McGregor Herbarium, University of Kansas for Douglas County, Kansas (C. Morse s.n.; October 2007).

AMARANTHACEAE Amaranth Family

Amaranthus albus L. - Tumbleweed. CH [K], CRW [K, G]. Native.

Amaranthus blitoides S. Wats. - Mat Amaranth. CH [K], CRW [K]. Native.

Amaranthus hybridus L. - Smooth Amaranth. CH [K], CRW [K, G]. Non-native.

Amaranthus retroflexus L. - Red-Root. CH [K], CRW [!, K, G]. Nonnative. CL.

Amaranthus spinosus L. - Spiny Amaranth. CH [K], CRW [!, G]. Native. Adventive. CL.

Amaranthus tuberculatus (Moq.) Sauer - Rough-Fruit Amaranth. $\mathrm{CH}$ [!, K], CRW [!, K, G]. Native. OP, OC, CL.

Bassia scoparia (L.) A.J. Scott - Mexican-Fireweed. CH [K], CRW [K]. Non-native. 
Chenopodiastrum simplex (Torr.) S. Fuentes, Uotila \& Borsch - GiantSeed Mock Goosefoot. CH [K], CRW [!, K]. Native. OC, CL.

Chenopodium album L. var. album - Lamb's-Quarters. CH [!, K], CRW [K]. Non-native. CL.

Chenopodium album L. var. missouriense (Aellen) Bassett \& Crompton - Lamb's-Quarters. CRW [!]. Native. OC. Crawford County Record (Pryer 6032).

Chenopodium berlandieri Moq. var. bushianum (Aellen) Cronquist - Pit-Seed Goosefoot. CRW [!]. Native. OC. Crawford County Record (Pryer 2820).

Chenopodium berlandieri Moq. var. zschackii (Murr) Murr - Pit-Seed Goosefoot. CH [K]. Native.

Chenopodium pratericola Rydb. - Desert Goosefoot. CH [!, K], CR [!]. Native. OC, CL. Crawford County Record (Pryer 6033).

Chenopodium standleyanum Aellen - Standley's Goosefoot. CH [! $\mathrm{K}], \mathrm{CRW}[!, \mathrm{K}, \mathrm{G}]$. Native. OP, CL.

Cycloloma atriplicifolium (Spreng.) Coult. - Winged-Pigweed. CH [K]. Native.

Dysphania ambrosioides (L.) Mosyakin \& Clemants - Mexican-Tea. $\mathrm{CH}[\mathrm{K}], \mathrm{CR}[\mathrm{K}]$. Non-native.

Froelichia floridana (Nutt.) Moq. var. campestris (Small) Fernald - Plains Snake-Cotton. CH [K], CRW [!]. Native. CL. Crawford County Record (Pryer 5333).

Froelichia gracilis (Hook.) Moq. - Slender Snake-Cotton. CH [K]. CRW [K]. Native.

Iresine rhizomatosa Standl. - Juda's-Bush. CH [!, K], CRW [!, K, G]. Native. OP, CL.

AMARYLLIDACEAE (Daffodil Family)

Leucojum aestivum L. subsp. aestivum - Summer Snowflake. CRW [!]. Non-native. CL. State Record (Pryer 27). Abundant and naturalized in Shlanger Park in Pittsburg, KS along creek banks. Scattered distribution across the country, mostly in the southern and eastern states. No collections reported from Oklahoma. A report from Boone County, Missouri, is the closest record.

Narcissus poeticus L. - Poet's Narcissus. CRW [!]. Non-native. CL. State Record (Pryer 41). Common throughout the county. This taxon has an eastern distribution, with the exception of Oregon and Washington. It has been reported in counties throughout Missouri and Arkansas, but not in Oklahoma. The closest report is in Newton County, Missouri. One specimen was located at McGregor Herbarium, University of Kansas for Douglas County, Kansas (C.C. Freeman 20037; April 2004).

Narcissus pseudonarcissus L. - Common Daffodil. CR [!]. Non-native. CL. Crawford County Record (Pryer 25).

ANACARDIACEAE (Sumac Family)

Rhus aromatica Aiton - Fragrant Sumac. CRW [G]. Gibson did not recognize varieties of this taxon at the time of publication.

Rhus aromatica Aiton var. aromatica - Fragrant Sumac. CH [!, K], CRW [!]. Native. OC, CL. Crawford County Record (Pryer 80, Pryer 4349)

Rhus aromatica Aiton var. serotina (Greene) Rehd. - Fragrant Sumac. CH [!], CRW [!, K]. Native. OC, CL. Cherokee County Record (Pryer 932, Pryer 4007).

Rhus copallinum L. - Winged Sumac. CH [!, K], CRW [!, K, G]. Native. $\mathrm{OP}, \mathrm{CL}$.

Rhus glabra L. - Smooth Sumac. CH [!, K], CRW [!, K]. Native. OP, $\mathrm{OC}, \mathrm{CL}$.

Toxicodendron pubescens P. Mill. - Eastern Poison-Oak. CH [K]. Native. Rare. Reported from only two counties in Kansas, but common in surrounding states. Found mostly in the southeastern portion of the United States.

Toxicodendron radicans (L.) Kuntze subsp. negundo (Greene) Gillis - Eastern Poison Ivy. CH [!, K], CRW [!, K, G]. Native. OC, CL.
Toxicodendron radicans (L.) Kuntze subsp. pubens (Engelm. ex S. Wats.) Gillis - Eastern Poison Ivy. CH [!], CRW [!]. Native. OP, CL. Crawford County Record (Pryer 1818). Cherokee County Record (Pryer 1303).

ANNONACEAE (Custard-Apple Family)

Asimina triloba (L.) Dunal - Common Pawpaw. CH [!, K], CRW [!, K, G]. Native. $\mathrm{OC}, \mathrm{CL}$.

\section{APIACEAE (Carrot Family)}

Ammoselinum butleri (Engelm. ex S. Wats.) Coult. \& Rose - Butler's Sand-Parsley. CH [K], CRW [K]. Native.

Anethum graveolens L. Dill. CRW [K]. Non-native.

Chaerophyllum procumbens (L.) Crantz var. procumbens - Spreading Chervil. CH [!, K], CRW [!, K, G]. Native. OP, CL. Gibson did not recognize varities of this taxon.

Chaerophyllum tainturieri Hook. - Hairy-Fruit Chervil. CH [!, K], CRW [!, K, G]. Native. OP, OC, CL.

Cicuta maculata L. - Spotted Water-Hemlock. CRW [G]. Gibson collection. He did not recognize varieties for this taxon.

Cicuta maculata L. var. angustifolia Hook. - Spotted Water-Hemlock. $\mathrm{CH}[\mathrm{K}]$. Native.

Cicuta maculata L. var. bolanderi (S. Wats.) G.A. Mulligan - Spotted Water-Hemlock. CH [K], CRW [!, K]. Native. CL.

Cicuta maculata L. var. maculata - Spotted Water-Hemlock. CH [K], CRW [!, K]. Native. CL.

Conium maculatum L. - Poison-Hemlock. CH [!, K], CRW [!, K]. Nonnative. $\mathrm{OC}, \mathrm{CL}$.

Cryptotaenia canadensis (L.) DC. - Canadian Honewort. CH [K], CRW [K, G]. Native.

Daucus carota L. - Queen Anne's-Lace. CH [!, K], CR [!, K, G]. Nonnative. Noxious. OC, CL.

Daucus pusillus Michx. - American Wild Carrot. CH [K]. Native. Rare. Reported from six counties in Kansas and common in surrounding states.

Erigenia bulbosa (Michx.) Nutt. - Harbinger-of-Spring. CH [K], CRW [K, G]. Native. Rare. Reported from four counties in Kansas. Common in Missouri and Arkansas, but considered rare in Oklahoma.

Eryngium leavenworthii Torr. \& A. Gray - Leavenworth's Eryngo. CR [!, K, G]. Native. OC.

Eryngium prostratum Nutt. ex DC. - Creeping Eryngo. CH [K]. Native. Rare. Reported in one county in Kansas, though this is the most northwestern report this taxon. Common in surrounding states.

Eryngium yuccifolium Michx. var. yuccifolium - Button Eryngo. $\mathrm{CH}$ [!, K], CRW [!, K]. Native. OP, CL.

Limnosciadium pinnatum (DC.) Mathias \& Constance - Tansy Dogshade. $\mathrm{CH}$ [K]. Native.

Lomatium foeniculaceum (Nutt.) Coult. \& Rose subsp. daucifolium (Torr. \& A. Gray) W.L. Theob. - Carrot-Leaf Desert-Parsley. CRW [!, K]. Native. OC.

Osmorhiza claytonii (Michx.) C.B. Clarke - Hairy Sweet-Cicely. CH [!]. CRW [!]. Native. OC, CL. Crawford County Record (Pryer 993, Pryer 1326, Pryer 2007, Pryer 2222). Cherokee County Record (Pryer 515b).

Osmorhiza longistylis (Torr.) DC. - Aniseroot. CH [K], CRW [!, K, G]. Native. $\mathrm{OP}, \mathrm{CL}$

Perideridia americana (Nutt. ex DC.) Reichenb. - Eastern Yampah. $\mathrm{CH}[!, \mathrm{K}], \mathrm{CRW}[!, \mathrm{K}, \mathrm{G}]$. Native. OP, CL.

Polytaenia nuttallii DC. - Nuttall's Prairie-Parsley. CH [!, K], CRW [!, $\mathrm{K}, \mathrm{G}]$. Native. OP, OC, CL.

Ptilimnium capillaceum (Michx.) Raf. - Herbwilliam. CRW [G]. Native. Ptilimnium nuttallii (DC.) Britton - Laceflower. CH [!, K], CRW [!, K, G]. Native. OC. CL. 
Sanicula canadensis L. var. canadensis - Canadian Black-Snakeroot. $\mathrm{CH}[!, \mathrm{K}], \mathrm{CRW}[!, \mathrm{K}]$. Native. OP, OC, CL.

Sanicula odorata (Raf.) K.M. Pryer \& L.R. Phillippe - Clustered BlackSnakeroot. CH [!, K], CRW [!, K, G]. Native. OC, CL.

Spermolepis inermis (Nutt. ex DC.) Mathias \& Constance - Red River Scaleseed. CH [K], CRW [K]. Native.

Taenidia integerrima (L.) Drude - Yellow-Pimpernel. CH [!, K], CRW [!]. Native. OP. OC. Crawford County Record (Pryer 5429).

Thaspium barbinode (Michx.) Nutt. - Hairy-Joint Meadow-Parsnip. $\mathrm{CH}[!, \mathrm{K}], \mathrm{CRW}[\mathrm{K}]$. Native. OP.

Torilis arvensis (Huds.) Link subsp. arvensis - Spreading HedgeParsley. CH [!, K], CRW [!, K, G]. Non-native. OC, CL.

Zizia aurea (L.) W.D.J. Koch - Golden Alexanders. CH [!, K], CRW [!, $\mathrm{K}, \mathrm{G}$. Native. OC, $\mathrm{CL}$.

\section{APOCYNACEAE (Dogbane Family)}

Amsonia tabernaemontana Walt. var. salicifolia (Pursh) Woods. Eastern Bluestar. CH [!, K], CRW [K, G]. Native. Rare. OP. Reported in five counties in Kansas and common in surrounding states.

Amsonia tabernaemontana Walt. var. tabernaemontana - Eastern Bluestar. CH [!, K], CRW [K]. Native. Rare. OP. Reported in four counties in Kansas, but common in surrounding states.

Apocynum cannabinum L. - Indian-Hemp. CH [!, K], CRW [!, K, G]. Native. OC. CL.

Apocynum $\times$ floribundum Greene (pro sp.) $-\mathrm{CH}[\mathrm{K}]$. Native.

Asclepias amplexicaulis Sm. - Clasping Milkweed. CH [K]. Native.

Asclepias incarnata L. subsp. incarnata - Swamp Milkweed. CH [!, K], CRW [!, K, G]. Native. CL. Gibson did not recognize subspecies of this taxon.

Asclepias longifolia Michx. var. hirtella (Pennell) B.L. Turner - LongLeaf Milkweed. CH [!, K], CRW [!, K, G]. Native. OP, OC, CL.

Asclepias meadiiTorr. ex A. Gray-Mead's Milkweed. CRW [K]. Native. Rare. Reported in twelve counties in Kansas. This taxon is considered rare across its distribution, which includes: Kansas, Missouri, Wisconsin, Illinois, and Indiana.

Asclepias purpurascens L. - Purple Milkweed. CH [!, K], CRW [!, K, G]. Native. OP, OC.

Asclepias quadrifolia Jacq. - Four-Leaf Milkweed. CH [!, K]. Native. Rare. OP. Only reported in Cherokee County, Kansas, but common in surrounding states.

Asclepias stenophylla A. Gray - Slim-Leaf Milkweed. CH [K], CRW [K, G]. Native.

Asclepias sullivantii Engelm. ex A. Gray - Prairie Milkweed. CH [!, K], CRW [!, K,]. Native. OC, CL.

Asclepias syriaca L. - Common Milkweed. CH [!, K], CRW [!, K, G]. Native. OC, CL.

Asclepias tuberosa L. subsp. interior Woods. - Butterfly Milkweed. $\mathrm{CH}$ $[!, \mathrm{K}], \mathrm{CRW}[!, \mathrm{K}, \mathrm{G}]$. Native. OP, OC, CL. Gibson did not recognize varieties of this taxon.

Asclepias verticillata L. - Whorled Milkweed. CH [!, K], CRW [!, K, G]. Native. OP, OC, CL.

Asclepias viridiflora Raf. - Green Comet Milkweed. CH [K], CRW [!, $\mathrm{K}, \mathrm{G}]$. Native. OC, CL.

Asclepias viridis Walt. - Green Antelope-Horn. CH [!, K], CRW [!, K, G]. Native. OC, CL.

Cynanchum laeve (Michx.) Pers. - Honeyvine. CH [K], CRW [!, K, G]. Native. Noxious. OC.

Gonolobus suberosus (L.) R. Br. var. granulates (Scheele) Krings \& Q.Y. Xiang - Angular-Fruit Anglepod. CH [!, K], CRW [G]. Native. Rare. OP. Only reported in Cherokee County, Kansas, but common in surrounding states.

Matelea decipiens (Alexander) Woods. - Oldfield Milkvine. CH [!, $\mathrm{K}]$. Native. Rare. OP. Reported in two counties in Kansas and common in surrounding states.

Vinca minor L. - Lesser Periwinkle. CR [K]. Non-native.

\section{AQUIFOLIACEAE (Holly Family)}

Ilex decidua Walt. - Deciduous Holly. CH [!, K], CRW [!, K, G]. Natie. CL. Ilex opaca Aiton var. opaca - American Holly. CRW [!]. Native. CL.

State Record (Pryer 3456). Older tree located at the bottom of a slew in a mined land wildlife area. Where this specimen was growing, it was unlikely to have been purposefully planted. This taxon is common throughout the southeastern United States, with scattered records throughout the southern half of Missouri. This taxon is considered rare in Oklahoma and common throughout Arkansas. The closest records are in Craig County, Oklahoma and Greene County, Missouri.

ARACEAE (Arum Family)

Arisaema dracontium (L.) Schott var. dracontium - Greendragon. $\mathrm{CH}$ [!, K], CRW [!, K, G]. Native. OP, OC, CL.

Arisaema triphyllum (L.) Schott subsp. triphyllum - Jack-in-the-Pulpit. $\mathrm{CH}[\mathrm{K}], \mathrm{CRW}[!, \mathrm{K}, \mathrm{G}]$. Native. OC.

Lemna aequinoctialis Welw. - Lesser Duckweed. CRW [K]. Native.

Lemna minor L. - Common Duckweed. CH [!, K], CRW [!, G]. Native. $\mathrm{OP}, \mathrm{OC}, \mathrm{CL}$.

Lemna minuta Kunth - Least Duckweed. CH [!]. Native. OP. Cherokee County Record (Pryer 188, Pryer 5778, Pryer 5783a).

Lemna obscura (Austin) Daubs. - Little Duckweed. CH [K]. Native. Lemna perpusilla Torr. - Minute Duckweed. CH [!, K], CRW [!, K]. Native. OP, $\mathrm{CL}$.

Spirodela polyrhiza (L.) Schleid. - Common Duckmeat. CH [!, K], CRW [K]. Native. OP.

Wolffia brasiliensis Wedd. - Brazilian Watermeal. CH [K]. Native. Rare. Reported in ten counties in Kansas and scattered across the state. Common in surrounding states, but with scattered distribution.

Wolffia columbiana Karst. - Columbian Watermeal. CH [K].

\section{ARALIACEAE (Ginseng Family)}

Hedera helix L. - English Ivy. CRW [!]. Non-native. CL. State Record (Pryer 6413). Abundant in a reclaimed mined land that is now used as a public walking area. This taxon has a wide distribution in the United States. It has scattered reports in Missouri and Arkansas and none in Oklahoma. The closest report is from McDonald County, Missouri. One specimen was located at McGregor Herbarium, University of Kansas for Douglas County, Kansas (C. Morse s.n.; December 2007).

ARISTOLOCHIACEAE (Birthwort Family)

Asarum canadense L. - Canadian Wild Ginger. CH [!, K], CRW [G]. Native. OP.

Endodeca serpentaria (L.) Raf. - Virginia-Snakeroot. CH [K]. Native. Isotrema tomentosum (Sims) H. Huber - Woolly Pipevine. CH [!]. Native. CL.

ASPARAGACEAE (Asparagus Family)

Asparagus officinalis L. - Asparagus. CH [K], CRW [!, K, G]. Nonnative. $\mathrm{CL}$.

\section{ASTERACEAE (Aster Family)}

Achillea millefolium L. - Common Yarrow. CH [!, K], CRW [!, K, G]. Native. OC, CL.

Ageratina altissima (L.) King \& H.E. Rob. var. altissima - White Snakeroot. CH [!, K], CRW [!, K, G]. Native. OP, OC, CL.

Ambrosia artemisiifolia L. - Annual Ragweed. CH [!, K], CRW [!, K, G]. Native. OP, OC, CL.

Ambrosia bidentata Michx. - Lance-Leaf Ragweed. CH [!, K], CRW [!, $\mathrm{K}, \mathrm{G}]$. Native. OP, OC, CL.

Ambrosia psilostachya DC. - Perennial Ragweed. CH [!, K], CRW [!, $\mathrm{K}, \mathrm{G}$. Native. OP, OC, CL.

Ambrosia trifida L. - Great Ragweed. CH [!, K], CRW [!, K, G]. Native. $\mathrm{OC}, \mathrm{CL}$. 
Amphiachyris dracunculoides (DC.) Nutt. - Prairie Broomweed. CH [K], CRW [!, K, G]. Native. OC.

Antennaria neglecta Greene - Field Pussytoes. CH [!, K], CRW [!, K, $\mathrm{G}$. Native. OP, CL.

Antennaria parlinii Fernald subsp. fallax (Greene) Bayer \& Stebbins - Parlin's Pussytoes. CH [!, K], CRW [K]. Native. OP.

Anthemis cotula L. - Stinking Chamomile. CH [K], CRW [K, G]. Nonnative. $\mathrm{OC}, \mathrm{CL}$.

Arctium minus (Hill) Bernh. - Lesser Burrdock. CH [K], CRW [!, K, G]. Non-native. OC, CL.

Arnoglossum atriplicifolium (L.) H. Rob. - Pale Indian-Plantain. $\mathrm{CH}$ [K], CRW [!, K]. Native. OC.

Arnoglossum plantagineum Raf. - Groove-Stem Indian-Plantain. $\mathrm{CH}$ [!, K], CRW [!, K, G]. Native. OC, CL.

Artemisia ludoviciana Nutt. subsp. ludoviciana - White Sagebrush. CRW [!, G]. Native. OC.

Artemisia mexicana Willd. ex Spreng. - Mexican Wormwood. $\mathrm{CH}$ $[\mathrm{K}], \mathrm{CRW}[\mathrm{K}]$. Native.

Bidens aristosa (Michx.) Britton - Bearded Beggarticks. CH [!, K], CRW [!, K, G]. Native. OC, CL.

Bidens bipinnata L. - Spanish-Needles. CH [!, K], CRW [!, K, G]. Native. OC, OP, CL.

Bidens cernua L. - Nodding Burr-Marigold. CRW [!]. Native. OC. Crawford County Record (Pryer 3650).

Bidens frondosa L. - Devil's-Pitchfork. CH [!, K], CRW [!, K]. Native. $\mathrm{OP}, \mathrm{CL}$.

Boltonia asteroides (L.) L'Hér. var. latisquama (A. Gray) Cronquist White Doll's Daisy. CH [!, K], CRW [!, K, G]. Native. CL.

Boltonia asteroides (L.) L'Hér. var. recognita (Fernald \& Griscom) Cronquist - White Doll's Daisy. CH [!, K], CRW [!, K, G]. Native. $\mathrm{OP}, \mathrm{CL}$.

Bradburia pilosa (Nutt.) Semple - Soft Bradbury-Bush. CH [!, K], CRW [G]. Native. OP.

Brickellia eupatorioides (L.) var. corymbulosa (Torr. \& A. Gray) Shinners - ShinnersFalse Boneset. CH [K], CRW [!, K, G]. Native. OC, CL.

Brickellia eupatorioides (L.) var. texana (Shinners) Shinners - Shinners False Boneset. CH [K], CRW [!, K]. CL.

Carduus nutans L. - Nodding Plumeless-Thistle. CH [!, K], CRW [!, K, G]. Non-native. CL.

Chaetopappa asteroides (Nutt.) DC. var. asteroides - Arkansas Leastdaisy. CH [K]. Native. Rare. Reported in six counties in Kansas. Common in Oklahoma and Texas, but rare in Missouri.

Cichorium intybus L. - Chicory. CH [K], CRW [!, K, G]. Non-native. $\mathrm{OC}, \mathrm{CL}$.

Cirsium altissimum (L.) Spreng. - Tall Thistle. CH [!, K], CRW [!, K, G]. Native. OP, OC, $\mathrm{CL}$.

Cirsium arvense (L.) Scop. - Canadian Thistle. CR [K]. Non-native. Noxious.

Cirsium discolor (Muhl. ex Willd.) Spreng. - Field Thistle. CH [!], CRW [!]. Native. OC, CL. Cherokee County Record (Pryer 5887), Crawford County Record (Pryer 2354, Pryer 2416, Pryer 2550, Pryer 2660, Pryer 2708, Pryer 2908, Pryer 3557, Pryer 3622).

Cirsium undulatum (Nutt.) Spreng. - Wavy-Leaf Thistle. CH [K], CRW [K]. Native.

Cirsium vulgare (Savi) Ten. - Bull Thistle. CH [!, K], CRW [!, K]. Nonnative. Noxious. $\mathrm{OC}, \mathrm{CL}$.

Conoclinium coelestinum (L.) DC. - Blue Mistflower. CH [!, K], CRW [K, G]. Native. OP.

Coreopsis grandiflora Hogg ex Sweet - Large-Flower Tickseed. $\mathrm{CH}$ $[!$, K], CRW [!, K, G]. Native. OP, OC, CL. Given that Flora of North America and Steyermark's Flora of Missouri do not recognize varieties for this species, none were identified to infraspecific level. Kartesz (2017) reports Coreopsis grandifloraHogg ex Sweet var. harveyana (A. Gray) in both counties.
Coreopsis lanceolata L. - Lance-LeafTickseed. CH [!]. Native. Rare. OP, CL. Cherokee County Record (Pryer 372, Pryer 3162). Reported in eight counties in Kansas and common in surrounding states. Coreopsis palmata Nutt. - Stiff Tickseed. CH [!, K], CRW [K]. Native. OP. Coreopsis pubescens Elliott var. pubescens - Star Tickseed. CH [K], CRW [K]. Native. Rare. Reported in two counties in Kansas and common in surrounding areas.

Coreopsis tinctoria Nutt. var. tinctoria - Golden Tickseed. CH [!, K], CRW [!, K, G]. Native. OC, CL.

Coreopsis tripteris L. - Tall Tickseed. CH [K]. Native. Rare. Reported in six counties in Kansas and common in surrounding states.

Crepis capillaris (L.) Wallr. - Smooth Hawk's-Beard. CRW [K]. Non-native.

Cyanus segetum Hill - Garden Cornflower. CH [P.H. Ireland s.n., May 19, 1966; Roxy Blessant 1, May 9, 1985], CRW [John D. Smith s.n., April 30, 1974; Ian Sangster 52, April 25, 1976; J.J. Williams 132, May 5, 1978]. Non-native. OP, CL. Gibson reported this taxon by the older name Centaurea cyanus $\mathrm{L}$.

Dracopis amplexicaulis (Vahl) Cass. - Clasping-Coneflower. CH [!, K], CRW [!, K, G]. Native. OC, CL.

Dyssodia papposa (Vent.) A.S. Hitchc. - Fetid-Marigold. CH [K], CRW [K, G]. Native.

Echinacea angustifolia DC. - Blacksamson. CH [!, K], CRW [G]. Native. OP.

Echinacea pallida (Nutt.) Nutt. var. pallida - Pale Purple-Coneflower. $\mathrm{CH}[!, \mathrm{K}], \mathrm{CRW}[!, \mathrm{K}, \mathrm{G}]$. Native. OC, CL.

Echinacea purpurea (L.) Moench. - Eastern Purple-Coneflower. CH [K], CRW [!]. Native. Rare. CL. Crawford County Record (Pryer 5323). Reported from 3 counties in Kansas (including this report) and common in surrounding area.

Eclipta prostrata (L.) L. - False Daisy. CH [!, K], CRW [K, G]. Native. OC, CL.

Elephantopus carolinianus Raeusch. - Carolina Elephant's-Foot. CH [!, K], CRW [!, K, G]. Native. OP, OC, CL.

Erechitites hieraciifolius (L.) Raf. ex DC. var. hieraciifolius - American Burnweed. CH [!, K], CRW [!, K, G]. Native. OP, CL.

Erigeron annuus (L.) Pers. - Eastern Daisy Fleabane. CH [!, K], CRW $[!, \mathrm{K}, \mathrm{G}]$. Native. OP, OC, CL.

Erigeron canadensis L. - Canadian Horseweed. CH [!, K], CRW [!, K, G]. Non-native. $\mathrm{OP}, \mathrm{CL}$.

Erigeron divaricatus Michx. - Dwarf Horseweed. CH [!, K], CRW [!, K, G]. Native. OP, OC, CL.

Erigeron philadelphicus L. var. philadelphicus - Philadelphia Fleabane. Native. OC, CL.

Erigeron pulchellus Michx. var. pulchellus - Robin's-Plantain. CH [!, K]. Native. Rare. OP. Reported in Cherokee County, Kansas and common in surrounding states.

Erigeron strigosus Muhl. ex Willd. var. strigosus - Prairie Fleabane. $\mathrm{CH}[!, \mathrm{K}], \mathrm{CRW}[!, \mathrm{K}, \mathrm{G}]$. Native. OP, OC, CL.

Erigeron tenuis Torr. \& A. Gray - Slender-Leaf Fleabane. CH [!, K], CRW [!, G]. Native. Rare. OP, OC, CL. Reported in five counties in Kansas and common in surrounding areas.

Eupatorium altissimum L. - Tall Thoroughwort. CH [!, K]. CRW [!, K, G]. Native. OP, OC, CL.

Eupatorium perfoliatum L. - Common Boneset. CH [!, K], CRW [!, K, G]. Native. OP, CL.

Eupatorium serotinum Michx. - Late-Flowering Thoroughwort. $\mathrm{CH}$ $[!, \mathrm{K}]$, CRW [!, K, G]. Native. OP, OC, CL.

Eurybia hemispherica (Alexander) Nesom - Prairie Wood-Aster. $\mathrm{CH}$ $[!, \mathrm{K}], \mathrm{CRW}[\mathrm{K}, \mathrm{G}]$. Native. OP, CL.

Euthamia graminifolia (L.) Nutt. - Flat-Top Goldentop. CH [!], CRW [!]. Native. Rare. CL. Cherokee County Record (Pryer 2937, Pryer 3386, Pryer 3686), Crawford County Record (Pryer 3700a, Pryer 6112, Pryer 6229). Reported in three counties in 
Kansas, including this report. Considered rare in Oklahoma and scattered throughout Missouri. Common in the northeastern United States.

Euthamia gymnospermoides Greene - Texas Goldentop. CH [!, K], CRW [!, K, G]. Native. CL.

Euthamia leptocephala (Torr. \& A. Gray) Greene ex Porter \& Britton - Bushy Goldentop. CR [!]. Native. CL. State Record. Crawford County Voucher (Pryer 1908). This taxon has a southern distribution in the United States and has been reported throughout Arkansas. It is considered rare in Oklahoma and only recorded in three southern counties and few counties in Missouri. The closest record is in Jasper County, Missouri.

Eutrochium purpureum (L.) E. Lamont var. holzingeri (Rydb.) E. Lamont - Sweet-Scented Joe-Pye-Weed. CH [K], CRW [!, K]. Native. OC, CL.

Eutrochium purpureum (L.) E. Lamont var. purpureum - SweetScented Joe-Pye-Weed. CH [!, K], CRW [G]. Native. OP.

Fleischmannia incarnata (Walt.) King \& H.E. Rob. - Pink SlenderThoroughwort. CH [!]. Native. OP, CL. State Record. Cherokee County Voucher (Pryer 3103, Pryer 3692b, Pryer 3916). This taxon has a southern distribution in the United States. It is scattered throughout Arkansas, eastern Oklahoma, and southern Missouri. The closest records are Ottawa County, Oklahoma and Newton County, Missouri.

Gaillardia pulchella Foug. var. pulchella - Firewheel. CH [!], CRW [!, K]. Native. OC, CL. Cherokee County Record (Pryer 923, Pryer 3102, Pryer 4987).

Galinsoga parviflora Cav. var. parviflora - Gallant-Soldier. CH [K], CRW [K]. Non-native.

Galinsoga quadriradiata Ruiz \& Pav. - Shaggy-Soldier. CH [K], CRW [G]. Non-native.

Gamochaeta argyrinea Nesom - Silvery Everlasting. CH [!, K]. Native. Rare. OP. Reported in only Cherokee County, Kansas. This taxon is rare in Missouri as well with reports from only two counties. However, it is common in Oklahoma and Arkansas.

Gamochaeta purpurea (L.) Cabrera - Spoon-Leaf Purple Everlasting. $\mathrm{CH}[!, \mathrm{K}], \mathrm{CRW}[!, \mathrm{K}, \mathrm{G}]$. Native. OP, OC, CL.

Grindelia lanceolata Nutt. var. lanceolata - Narrow-Leaf Gumweed. $\mathrm{CH}[\mathrm{K}], \mathrm{CRW}[!, \mathrm{K}, \mathrm{G}]$. Native. OC, CL.

Helenium amarum (Raf.) H. Rock var. amarum - Yellowdicks. CH [!, $\mathrm{K}], \mathrm{CRW}[\mathrm{K}, \mathrm{G}]$. Native. Adventive. OP, CL.

Helenium autumnale L. - Autumn Sneezeweed. CH [K], CRW [K]. Native.

Helenium flexuosum Raf. - Purple-Head Sneezeweed. CH [!, K], CRW $[!, K, G]$. Native. Rare. OP, CL. Reported in six counties in Kansas and common in surrounding states.

Helianthus annuus L. - Common Sunflower. CH [!, K], CRW [!, K, G]. Native. OC, CL.

Helianthus grosseserratus Martens - Saw-Tooth Sunflower. CH [!, K], CRW [!, K, G]. Native. OP, CL.

Helianthus hirsutus Raf. - Whiskered Sunflower. CH [!, K], CRW [K]. Native. OP, CL.

Helianthus $\times$ kellermanii Britton (pro sp.) - CRW [K]. Native.

Helianthus maximiliani Schrad. - Maximilian Sunflower. CH [!, K], CRW [!, K, G]. Native. OP, OC, CL.

Helianthus mollis Lam. - Ashy Sunflower. CH [!, K], CRW [!, K, G]. Native. OP, OC, CL.

Helianthus pauciflorus Nutt. subsp. pauciflorus - Stiff Sunflower. $\mathrm{CH}$ $[\mathrm{K}], \mathrm{CRW}[\mathrm{K}, \mathrm{G}]$. Native.

Helianthus petiolaris Nutt. subsp. petiolaris - Prairie Sunflower. $\mathrm{CH}$ [!, K]. CRW [!]. Native. OC, CL. Crawford County Record (Pryer 1395, Pryer 2536, Pryer 2539, Pryer 2716, Pryer 2878, Pryer 2879, Pryer 3311, Pryer 3312, Pryer 3397, Pryer 3581, Pryer 6286).

Helianthus salicifolius A. Dietr. - Willow-Leaf Sunflower. CH [!, K], CRW [!, K, G]. Native. OC.
Helianthus strumosus L. - Pale-Leaf Woodland Sunflower. CH [!, K], CRW [!, G]. Native. Rare. OP, OC.

Helianthus tuberosus L. - Jerusalem-Artichoke. CH [!, K], CRW [!, K, G]. Native. OP, OC, CL.

Heliopsis helianthoides (L.) var. scabra (Dunal) Fernald - SweetSmooth Oxeye. CH [!, K], CRW [!, K, G]. Native. OP, CL.

Hieracium gronovii L. - Queendevil. CH [!, K], CRW [K]. Native. Rare. OP. Reported from nine counties in Kansas, but common in surrounding states.

Hieracium longipilum Torr. ex Hook. - Hairy Hawkweed. CH [K], CRW [!, K, G]. Native. CL.

Hieracium scabrum Michx. - Rough Hawkweed. CH [K]. Natie. Rare. Reported only from Cherokee County, Kansas. This taxon is also considered rare in Oklahoma and Arkansas, but common in Missouri.

Hymenopappus scabiosaeus L'Hér. var. corymbosus (Torr. \& A. Gray) B.L. Turner - Carolina Woollywhite. CH [K], CRW [K]. Native.

lonactis linariifolia (L.) Greene - Flax-Leaf Ankle-Aster. CH [K]. Native. Rare. Reported only from Cherokee County, Kansas; considered rare in Oklahoma, but common in Missouri and Arkansas.

Iva annua L. - Annual Marsh-Elder. CH [!, K], CRW [!, K, G]. Native. $\mathrm{OC}, \mathrm{CL}$.

Iva asperifolia Less. var. angustifolia (Nutt. ex DC.) B.L. Turner Pensacola Marsh-Elder. CH [K]. Native. Rare. Reported in three counties in Kansas. Rare in surrounding areas, though considered common for the state of Oklahoma.

Krigia cespitosa (Raf.) Chambersvar. cespitosa - Weedy DwarfDandelion. CH [!, K], CRW [!, K, G]. Native. OP, CL.

Krigia dandelion (L.) Nutt. - Potato Dwarf-Dandelion. CH [K], CRW $[!, \mathrm{K}, \mathrm{G}]$. Native. OC, CL.

Krigia occidentalis Nutt. - Western Dwarf-Dandelion. CH [K], CRW [G]. Native. Rare. Reported in five counties in Kansas and common in Missouri and Oklahoma; considered rare in Arkansas but with a southern distribution statewide.

Lactuca canadensis L. - Canadian Blue Lettuce. CH [!, K], CRW [!, K, G]. Native. OP, OC, CL.

Lactuca floridana (L.) Gaertn. - Woodland Lettuce. CH [!, K], CRW [!, $\mathrm{K}, \mathrm{G}]$. Native. OP, OC, CL.

Lactuca saligna L. - Willow-Leaf Lettuce. CH [K], CRW [!, K]. Nonnative. $\mathrm{CL}$.

Lactuca serriola L. - Prickly Lettuce. CH [!, K], CRW [!, K, G]. Nonnative. $\mathrm{CL}$.

Leucanthemum vulgare Lam. - Ox-Eye Daisy. CH [!, K], CRW [!, K, G]. Non-native. Noxious. OC, CL.

Liatris aspera Michx. - Tall Gayfeather. CH [!, K], CRW [!, K, G]. Native. $\mathrm{OC}, \mathrm{CL}$.

Liatris hirsuta Rydb. - Calcareous Gayfeather. CH [!, K]. Native. OP.

Liatris punctata Hook. var. mucronata (DC.) B.L. Turner - Dotted Gayfeather. CH [!, K], CRW [!, G]. Native. OP, OC, CL. Gibson reported Liatris punctata, and did not recognize variations within this taxon at the time of his thesis. I did verify that his specimens are in fact var. mucronata.

Liatris punctata Hook. var. punctata - Dotted Gayfeather. CRW [K]. Native.

Liatris pycnostachya Michx. var. pycnostachya - Cat-Tail Gayfeather. CH [!, K], CRW [!, K, G]. Native. OP, CL. Gibson reported Liatris pycnostachya and did not recognize variations within this taxon at the time of his thesis.

Marshallia caespitosa Nutt. ex DC. var. caespitosa - Puffballs. $\mathrm{CH}$ [K]. Native.

Matricaria chamomilla L. - Wild Chamomile. CRW [K, G]. Non-native. Matricaria discoidea DC. - Pineapple-Weed. CH [!]. Native. CL. Cherokee County Record (Pryer 645).

Nabalus asperus (Michx.) Torr. \& A. Gray - Rough Rattlesnake-Root. $\mathrm{CH}[\mathrm{K}], \mathrm{CRW}[!, \mathrm{K}, \mathrm{G}]$. Native. CL. 
Packera glabella (Poir.) C. Jeffrey - Cress-Leaf Groundsel. CH [K], CRW [!, K, G]. Native. Rare. Reported in ten counties in Kansas and common in surrounding states.

Packera obovata (Muhl. ex Willd.) W.A. Weber \& A. Löve - Round-Leaf Groundsel. CH [!, K], CRW [!, K, G]. Native. OP, OC, CL.

Packera plattensis (Nutt.) W.A. Weber \& A. Löve - Prairie Groundsel. $\mathrm{CH}[!, \mathrm{K}], \mathrm{CRW}[!, \mathrm{K}]$. Native. OP, CL.

Parthenium integrifolium L. - Wild Quinine. CH [!, K]. Native. OP, CL.

Pluchea camphorata (L.) DC. - Plowman's-Wort. CRW [!, K]. Native. Rare. CL. Reported in three counties in Kansas, but common in surrounding area. Found mostly in southern regions including Oklahoma and Arkansas.

Polymnia canadensis L. - White-Flower Leafcup. CH [K]. Native.

Pseudognaphalium obtusifolium (L.) Hilliard \& Burtt subsp. obtusifolium - Blunt-Leaf Rabbit-Tobacco. CH [K], CRW [!, K, G]. Native. OP, CL.

Pyrrhopappus carolinianus (Walt.) DC. - Carolina Desert-Chicory. $\mathrm{CH}[!, \mathrm{K}], \mathrm{CRW}[!, \mathrm{K}, \mathrm{G}]$. Native. OP, OC, CL.

Ratibida columnifera (Nutt.) Woot. \& Standl. - Red-Spike MexicanHat. CH [!, K], CRW [!, K, G]. Native. CL.

Ratibida pinnata (Vent.) Barnh. - Gray-Head Mexican-Hat. CH [!, K], CRW [!, K, G]. Native. OP, OC, CL.

Rudbeckia grandiflora (Sweet) K.C. Gmel. ex DC. var. grandiflora Rough Coneflower. CH [K], CRW [!, K]. Native. Rare. CL. Reported in four counties in Kansas, but common to the south in Oklahoma and Arkansas; considered rare in Missouri.

Rudbeckia hirta L. var. hirta - Black-Eyed-Susan. CH [!, K], CRW [!, $\mathrm{K}, \mathrm{G}$. Native. OP, OC, CL. Gibson did not recognize varieties for this taxon.

Rudbeckia laciniata L. var. laciniata - Green-Head Coneflower. CH [!, K], CRW [!, K, G]. Natifve. OP, OC, CL. Gibson did not recognize varieties for this taxon.

Rudbeckia subtomentosa Pursh - Sweet Coneflower. CH [K], CRW $[!, \mathrm{K}, \mathrm{G}]$. Native. CL.

Rudbeckia triloba L. var. triloba - Brown-Eyed-Susan. CH [!, K], CRW $[!, \mathrm{K}]$. Native. OP, OC, CL.

Senecio hieraciifolius L. var. hieraciifolius - American Burnweed. CH $[!, \mathrm{K}], \mathrm{CRW}[!, \mathrm{K}, \mathrm{G}]$. Native. OP, CL.

Silphium integrifolium Michx. var. integrifolium - Entire-Leaf Rosinweed. CH [K], CRW [!, K, G]. Native. OC, CL. Gibson did not recognize varieties for this taxon.

Silphium integrifolium Michx. var. laeve Torr. \& A. Gray - Entire-Leaf Rosinweed. CH [K]. Native.

Silphium laciniatum L. - Compassplant. CH [!, K], CRW [!, K, G]. Native. OC, CL.

Silphium perfoliatum var. perfoliatum - Cup-Plant. CH [!, K], CRW [!, K, G]. Native. OC. CL. Gibson did not recognize varieties for this taxon.

Smallanthus uvedalia (L.) Mack. ex Small - Bear's-Foot. CH [K]. Native. Rare. Only reported from Cherokee County, Kansas, but common in surrounding states.

Solidago altissima L. var. altissima - Tall Goldenrod. CH [!], CRW [!, K]. Native. OC, CL. Crawford County Record (Pryer 2588, Pryer 2691, Pryer 2711, Pryer 2851, Pryer 2936, Pryer 2944, Pryer 3310, Pryer 3315, Pryer 3385, Pryer 3394, Pryer 3535, Pryer 3555, Pryer 3615, Pryer 5893, Pryer 5921, Pryer 6069, Pryer 6098, Pryer 6232). Gibson did not recognize varieties for this taxon.

Solidago altissima L. var. gilvocanescens (Rydb.) Semple - Tall Goldenrod. CH [!], CRW [!, K, G]. Native. OC, CL. Cherokee County Record (Pryer 5855). Gibson recognized an older name for this taxon, Solidago canadensis var. gilvocanescens.

Solidago arguta Aiton var. boottii (Hook.) E.J. Palmer \& Steyerm. Atlantic Goldenrod. CH [!, K]. Native. Rare. OP. Reported from Cherokee County, Kansas, but common in the surrounding areas.
Solidago arguta Aiton var. caroliniana A. Gray - Atlantic Goldenrod. $\mathrm{CH}$ [!]. Native. OP. State Record. Cherokee County Voucher (Pryer 6303). This taxon has a southeastern distribution in the United States. It has been reported in several southern counties in Missouri but for Arkansas or Oklahoma. The closest record is Barry County, Missouri.

Solidago delicatula Small - Smooth Elm-Leaf Goldenrod. CH [K]. CRW [!, K]. Native. OP, CL.

Solidago gigantea Aiton - Late Goldenrod. CH [!, K]. CRW [!, K, G]. Native. OP, OC, CL.

Solidago missouriensis Nutt. - Missouri Goldenrod. CH [!, K], CRW [!, K, G]. Native. OP, CL.

Solidago nemoralis Aiton var. longipetiolata (Mack. \& Bush) E.J. Palmer \& Steyerm. - Goldenrod. CH [!], CRW [!, G]. Native. OP, CL. Cherokee County Record (Pryer 3015, Pryer 6347).

Solidago nemoralis Aiton var. nemoralisA. Gray - Goldenrod. CH [!, $\mathrm{K}], \mathrm{CRW}[!, \mathrm{K}]$. Native. OP, OC, CL.

Solidago petiolaris Aiton var. angusta (Torr. \& A. Gray) A. Gray Downy Ragged Goldenrod. CH [!, K], CRW [K]. Native. OP.

Solidago radula Nutt. - Western Rough Goldenrod. CH [!, K], CRW [!]. Native. CL. Crawford Country Record (Pryer 3434).

Solidago rigida L. subsp. rigida - Hard-Leaf Flat-Top-Goldenrod. $\mathrm{CH}$ [K], CRW [!, K]. Native. OC, CL. Gibson did not recognize subspecies for this taxon.

Solidago speciosa Nutt. var. rigidiuscula Torr. \& A. Gray - Showy Goldenrod. $\mathrm{CH}[\mathrm{K}], \mathrm{CRW}[\mathrm{K}, \mathrm{G}]$. Native.

Solidago ulmifolia Muhl. Ex Willd. var. ulmifolia - Elm-Leaf Goldenrod. CH [!, K], CRW [K, G]. Native. Gibson did not recognize varieties for this taxon.

Sonchus asper (L.) Hill - Spiny-Leaf Sow-Thistle. CH [!, K], CRW [!, K, G]. Non-native. OP, OC, CL.

Sonchus oleraceus L. - Common Sow-Thistle. CRW [!]. Non-native. CL. Cherokee County Record (Pryer 1137).

Symphyotrichum anomalum (Engelm.) Nesom - Many-Ray American-Aster. CH [!, K]. Native. Rare. OP. Reported in two counties in Kansas, but common in surrounding areas.

Symphyotrichum cordifolium (L.) Nesom - Common Blue AmericanAster. CH [!], CRW [!]. Native. OP, OC. Crawford County Record (Pryer 6273). Cherokee County Record (Pryer 2961, Pryer 3228).

Symphyotrichum drummondii (Lindl.) Nesom var. drummondii Drummond's American-Aster. CH [!, K], CRW [!, K, G]. Native. $\mathrm{OP}, \mathrm{CL}$.

Symphyotrichum drummondii (Lindl.) Nesom var. texanum (Burgess) Nesom - Drummond's American-Aster. CH [!], CRW [!]. Native. OP, OC, CL. Cherokee County Record (Pryer 2959, Pryer 2968, Pryer 2987, Pryer 3189, Pryer 6293, Pryer 6315), Crawford County Record (Pryer 6164, Pryer 6173, Pryer 6277).

Symphyotrichum ericoides (L.) Nesom var. ericoides - White Heath American-Aster. CH [!, K], CRW [!, K, G]. Native. OC, CL. Gibson did not recognize varieties for this taxon. He reported Aster ericoides.

Symphyotrichum lanceolatum (Willd.) Neson subsp. interior (Wieg.) Nesom - White Panicled American-Aster. CH [!, K], CRW [!]. Native. OP, CL. Crawford County State Record (Pryer 6408).

Symphyotrichum lanceolatum (Willd.) Neson subsp. lanceolatum White Panicled American-Aster. CH [!, K], CRW [!, K, G]. Native. $\mathrm{OP}, \mathrm{OC}, \mathrm{CL}$.

Symphyotrichum lateriflorum (L.) A.\& D. Löve - Farewell-Summer. $\mathrm{CH}[\mathrm{K}]$. Native. Rare. Reported from four counties in Kansas but common in surrounding states.

Symphyotrichum novae-angliae (L.) Nesom - New England American-Aster. $\mathrm{CH}[\mathrm{K}]$. Native.

Symphyotrichum oblongifolium (Nutt.) Nesom - Aromatic AmericanAster. CH [K], CRW [!, K]. Native. CL. 
Symphyotrichum ontarionis (Wieg.) Nesom var. ontarionis - Ontario American-Aster. $\mathrm{CH}[\mathrm{K}], \mathrm{CRW}[\mathrm{K}, \mathrm{G}]$. Native.

Symphyotrichum oolentangiense (Riddell) Nesom - Sky-Blue American-Aster. CH [!, K], CRW [!, K]. Native. OP, CL.

Symphyotrichum parviceps (Burgess) Nesom - Small-Head American-Aster. $\mathrm{CH}[\mathrm{K}]$. Native.

Symphyotrichum patens (Aiton) Nesom var. patens - Late Purple American-Aster. CH [!, K]. Native. OP.

Symphyotrichum patens (Aiton) Nesom var. patentissimum (Lindl. ex DC.) Nesom - Late Purple American-Aster. CH [!, K], CRW [K]. Native. OP.

Symphyotrichum pilosum (Willd.) Nesom var. pilosum - White Oldfield American-Aster. CH [!, K], CRW [!, K, G]. Native. OC, CL.

Symphyotrichum praealtum (Poir.) Nesom var. praealtum - WillowLeaf American-Aster. CH [!, K], CRW [!, K, G]. Native. OC, CL.

Symphyotrichum turbinellum (Lindl.) Nesom - Smooth Violet Prairie American-Aster. CH [!, K]. Native. Rare. OP. Only reported from Cherokee County, Kansas, but common in surrounding states.

Symphyotrichum urophyllum (Lindl. ex DC.) Nesom - White Arrow American-Aster. CH [K], CRW [!]. Native. CL. Crawford County Record (Pryer 3539).

Tanacetum vulgare L. - Common Tansy. CRW [K]. Non-native. Noxious.

Taraxacum erythrospermum Andrz. ex Bess. - Red-Seed Dandelion. $\mathrm{CH}[\mathrm{K}], \mathrm{CRW}[\mathrm{K}, \mathrm{G}]$. Non-native

Taraxacum officinale G.H.Weber ex Wiggers - Common Dandelion. $\mathrm{CH}[!, \mathrm{K}], \mathrm{CRW}[!, \mathrm{K}, \mathrm{G}]$. Native. CL.

Tragopogon dubius Scop. - Meadow Goat's-Beard. CH [!, K], CRW [!, $\mathrm{K}, \mathrm{G}]$. Non-native. OP, OC, CL.

Verbesina alternifolia (L.) Britton ex Kearney - Wingstem. CH [!, K], CRW [!, K, G]. Native. OC, CL.

Verbesina encelioides (Cav.) Benth. \& Hook. f. ex A. Gray - Golden Crownbeard. CRW [!]. Native. CL. Crawford County Record (Pryer 3877).

Verbesina helianthoides Michx. - Gravelweed. CH [!, K], CRW [!, K, G]. Native. Rare. OP, CL. In Kansas reported for only six counties in the southeast; becoming more nd common to the east.

Verbesina virginica L. - White Crownbeard. CH [!, K], CRW [!, K, G]. Native. $\mathrm{OC}, \mathrm{CL}$.

Vernonia arkansana DC. - Arkansas Ironweed. CH [!, K], CRW [!, K, G]. Native. OP, OC, CL.

Vernonia baldwinii Torr. - Western Ironweed. CH [!, K], CRW [!, K, G]. Native. $\mathrm{OP}, \mathrm{OC}, \mathrm{CL}$.

Vernonia fasciculata Michx. - Prairie Ironweed. CH [!, K], CRW [K, G]. Native. CL.

Vernonia gigantea (Walt.) Trel. ex Branner \& Coville - Giant Ironweed. $\mathrm{CH}[\mathrm{K}]$. Native. Rare. Reported in three counties in Kansas, but common in surrounding states.

Vernonia missurica Raf. Missouri Ironweed. CH [K], CRW [!, K, G]. Native. Rare. CL. Reported in four counties in Kansas, but common in surrounding states.

Xanthium strumarium L. - Rough Cockleburr. CH [!, K], CRW [!, K, G]. Native. Noxious. OC, CL.

BALSAMINACEAE (Touch-Me-Not Family)

Impatiens capensis Meerb. - Spotted Touch-Me-Not. CH [!, K], CRW $[!, \mathrm{K}, \mathrm{G}]$. Native. OP, OC, CL.

Impatiens pallida Nutt. - Pale Touch-Me-Not. CH [!, K], CRW [K, G]. Native. OP.

BERBERIDACEAE (Barberry Family)

Podophyllum peltatum L. - May-Apple. CH [!, K], CRW [!, K, G]. Native. OP, OC, CL.

BETULACEAE (Birch Family)

Alnus serrulata (Aiton) Willd. - Brookside Alder. $\mathrm{CH}[\mathrm{K}]$. Native.
Betula nigra L. - River Birch. CH [!, K], CRW [!, K, G]. Native. OP, CL. Plant observed in Crawford County but not collected.

Corylus Americana Marshall - American Hazelnut. CH [K], CRW [!, $\mathrm{K}, \mathrm{G}]$. Native. OC.

Ostrya virginiana (P. Mill.) K. Koch - Eastern Hop-Hornbeam. CH $[\mathrm{K}], \mathrm{CRW}[\mathrm{K}]$. Native.

BIGNONIACEAE (Trumpet-Creeper Family)

Campsis radicans (L.) Seem. ex Bureau -Trumpet-Creeper. CH [!, K], CRW [!, K, G]. Native. OP, CL.

Catalpa bignonioides Walt. - Southern Catalpa. CH [!], CRW [!, G]. Native. Adventive. CL. Cherokee County Record (Pryer 4956, Pryer 4963, Pryer 5041)

Catalpa ovata G. Don - Chinese Catalpa. CRW [!]. Non-native. CL. State Record. Crawford County Voucher (Pryer 2085). One tree located in the middle of a hay field and suckering. Present two years in a row. Reported from few counties nationwide, in mostly northeastern states. Reported only from two counties in Missouri, Boone and Crawford. The Missouri records are the closest reports.

Catalpa speciosa Warder ex Engelm. - Northern Catalpa. CH [K], CRW [!, K, G]. Native. Adventive. OC, CL.

BORAGINACEAE (Borage Family)

Buglossoides arvensis (L.) I.M. Johnst. - Corn-Gromwell. CH [!, K], CRW [!, K, G]. Non-native. OP, CL.

Ellisianyctelea (L.) L. - Aunt Lucy. CH [K], CRW [!, K, G]. Native. OP, CL. Hackelia virginiana (L.) I.M. Johnst. - Beggar's-Lice. CH [K], CRW [!, $\mathrm{K}, \mathrm{G}]$. Native. OC.

Heliotropium indicum L. - Indian Heliotrope. CH [!, K], CRW [!, K, G]. Native. Adventive. OP.

Heliotropium tenellum (Nutt.) Torr. - Pasture Heliotrope. CRW [!, K, G]. Native. OC.

Hydrophyllum virginianum L. var. virginianum - Shawnee-Salad. $\mathrm{CH}[\mathrm{K}], \mathrm{CRW}[\mathrm{K}, \mathrm{G}]$. Native. Gibson did not recognize varieties of this taxon.

Lithospermum canescens (Michx.) Lehm. - Hoary Puccoon. CH [K], CRW [K, G]. Native.

Lithospermum incisum Lehm. - Fringed Gromwell. CH [!, K], CRW [!, $\mathrm{K}, \mathrm{G}]$. Native. OC, CL.

Lithospermum occidentale (Mack.) Weakley, Witsell \& D. Estes Western Marbleseed. CRW [!, K, G]. Native. OC.

Myosotis macrosperma Engelm. - Large-Seed Forget-Me-Not. CRW [!]. Native. CL. State Record. Crawford County Vouchers (Pryer 598, Pryer 987, Pryer 4223, Pryer 4270). This taxon is common throughout the south and eastern United States, including the 4-State region. The closest reports are Barton and Jasper counties, Missouri.

Myosotis verna Nutt. - Spring Forget-Me-Not. CH [!, K], CRW [!, K, G]. Native. OP, CL.

Phacelia gilioides Brand - Brand's Scorpion-Weed. CH [K], CRW [K, G]. Native.

Phacelia hirsuta Nutt. - Fuzzy Scorpion-Weed. CH [K], CRW [!, K, G]. Native. OC.

BRASSICACEAE (Mustard Family)

Abdra brachycarpa (Nutt. ex Torr. \& A. Gray) Greene - Smooth CrossHair Cress. CH [!, K], CRW [!, K, G]. Native. OC, CL.

Alliaria petiolata (Bieb.) Cavara \& Grande - Garlic-Mustard. CRW [!]. Non-native. Noxious. OC, CL. Crawford County Record (Pryer 4261, Pryer 4313, Pryer 4541).

Arabidopsis thaliana (L.) Heynh. - Thalecress. CH [K]. Non-native.

Arabis pycnocarpa M. Hopkins var. adpressipilis M. Hopkins - Hairy Eared Rockcress. CH [K]. Native. Rare. Only reported from Cherokee County, Kansas, but common in Missouri; considered rare in Arkansas and has not been reported in Oklahoma. 
Barbarea vulgaris W.T. Aiton - Garden Yellow-Rocket. CH [!, K], CRW [!, K, G]. Non-native. OC, CL.

Borodinia canadensis (L.) P.J. Alexander \& Windham - Sicklepod. CH [K], CRW [K, G]. Native.

Borodinia dentata (Raf.) P.J. Alexander \& Windham - Short's False Rockcress. CH [!, K]. Native. OP.

Borodinia laevigata (Muhl. ex Willd.) P.J. Alexander \& Windham Smooth False Rockcress. CH [K], CRW [!, K]. Native. Rare. OP, OC. Reported in three counties in Kansas, but common in surrounding states.

Borodinia missouriensis (Greene) P.J. Alexander \& Windham - Green False Rockcress. CH [!, K]. Native. Rare. OP. Only reported in Cherokee County, Kansas, but common in surrounding states.

Brassica juncea (L.) Czern. - Chinese Mustard. CRW [G]. Non-native. Noxious.

Brassica rapa L. var. rapa - Rape (Canola). CRW [!]. Non-native. Noxious. CL. Crawford County Record (Pryer 100, Pryer 110, Pryer 4257).

Camelina microcarpa Andrz. ex DC. - Little-Pod False Flax. CH [K], CRW [K, G]. Non-native.

Capsella bursa-pastoris (L.) Medik. - Shepherd's-Purse. CH [!, K], CRW $[!, \mathrm{K}, \mathrm{G}]$. Non-native. OC, CL.

Cardamine bulbosa (Schreb. ex Muhl.) B.S.P. - Bulbous Bittercress. $\mathrm{CH}[\mathrm{K}]$. Native. Rare. Reported from five counties in Kansas, but common in surrounding states.

Cardamine concatenata (Michx.) Sw. - Cut-Leaf Toothwort. CH [!, K], CRW [!, K, G]. Native. OP, OC, CL.

Cardamine hirsuta L. - Hairy Bittercress. CH [!], CRW [!]. Non-native. OP, OC. State Record. Cherokee County Vouchers (Pryer 4116, Pryer 4128, Pryer 4207, Pryer 4208). Crawford County voucher (Pryer 4053, Pryer 4066). This taxon is scattered throught the United States and most common throught the southeast. It is commonly reported in the 4-state region. The closest reports are from Barry County, Missouri and Osage County, Oklahoma. This taxon was also collected in Leavenworth County, Kansas and is accessioned in the McGregor Herbarium at the University of Kansas (KANU 395956 - C.C. Freeman 25760).

Cardamine parviflora L. - Sand Bittercress. CH [!, K], CRW [!, K, G]. Native. OC, CL.

Cardamine pensylvanica Muhl. ex Willd. Quaker Bittercress. CH [K], CRW [G]. Native.

Chorispora tenella (Pallas) DC. - Crossflower. CRW [!]. Non-native. OC. Crawford County Record (Pryer 4857).

Descurainia pinnata (Walt.) Britton subsp. brachycarpa (Richardson) Detling - Western Tansy-Mustard. CH [!], CRW [!, G]. Native. OP, OC, CL. Crawford County Record (Pryer 556, Pryer 4291, Pryer 4334, Pryer 4391, Pryer 4514, Pryer 4515, Pryer 4567).

Draba brachycarpa Nutt. ex Torr. \& A. Gray - Short-Pod WhitlowGrass. Ch [!, K], CRW [!, K, G]. Native. OC, CL.

Draba cuneifolia Nutt. ex Torr. \& A. Gray var. cuneifolia - WedgeLeaf Whitlow-Grass. CH [K], CRW [!, K, G]. Native. OC. Gibson did not recognize varities of this taxon. Crawford County Record (Pryer 4331).

Draba reptans (Lam.) Fernald - Carolina Whitlow-Grass. CH [K], CRW [!, G]. Native. OC.

Erysimum capitatum (Dougl. ex Hook.) Greene - Sand-Dune Wallflower. CRW. Native. State Record. Collected in 1998 along the roadside of Highway 160. Crawford County Voucher (Timme 15028). This taxon is common in western states and adventive in the Great Plains region. The closest report is Benton County, Arkansas, Barry County, Missouri, and Rogers County, Oklahoma.

Erysimum repandum L. - Spreading Wallflower. $\mathrm{CH}[\mathrm{K}], \mathrm{CRW}[!, \mathrm{K}, \mathrm{G}]$. Non-native. Noxious. OC, CL.
Hesperis matronalis L. - Mother-of-the-Evening. CH [!], CRW [!, K]. Native. OC, CL. Cherokee County Record (Pryer 629).

lodanthus pinnatifidus (Michx.) Steud. - Purple-Rocket. CH [K], CRW [!, K, G]. Native. CL.

Lepidium campestre (L.)W.T. Aiton - Cream-Anther Field Pepperwort. CH [!], CRW [!]. Non-native. Cherokee County Record (Pryer 433), Crawford County Record (Timme 17044; Snow 10769; Pryer 4271, Pryer 4507).

Lepidium densiflorum Schrad. - Miner's Pepperwort. CH [!, K], CRW [!, K, G]. Native. OC.

Lepidium virginicum $L$. subsp. virginicum - Poorman's-Pepperwort. $\mathrm{CH}[!, \mathrm{K}], \mathrm{CRW}[!, \mathrm{K}, \mathrm{G}]$. Native. OP, OC, CL.

Nasturtium officinale W.T. Aiton - Watercress. CH [K]. Non-native. Physaria gracilis (Hook.) O'Kane \& Al-Shehbaz subsp. gracilis Spreading Bladderpod. CRW [!, G]. Native. CL.

Planodes virginica (L.) Greene - Virginia Winged Rockcress. CH [!, K], CRW [!, K, G]. Native. OP, OC, CL.

Rorippa palustris (L.) Bess. subsp. palustris - Bog Yellowcress. CH [!, K]. CRW [!]. Native. OP, OC, CL. Crawford County Record (Pryer 592, Pryer 602, Pryer 4550).

Rorippa sessiliflora (Nutt.) A.S. Hitchc. - Stalkless Yellowcress. CH [!, $\mathrm{K}]$, CRW [!, K]. Native. CL.

Rorippa sinuata (Nutt.) A.S. Hitchc. - Spreading Yellowcress. CRW [K]. Native.

Selenia aurea Nutt. - Golden Selenia. CH [K], CRW [K, G]. Native

Sinapis arvensis L. - Corn-Mustard. CH [K], CRW [K]. Non-native. Noxious.

Sisymbrium altissimum L. -Tall Hedge-Mustard. CRW [G]. Non-native. Sisymbrium officinale (L.) Scop. - Hedge-Mustard. CH [K], CRW [!, $\mathrm{K}, \mathrm{G}]$. CL.

Thlaspi arvense L. - Field Pennycress. CH [!, K], CRW [!, K, G]. Nonnative. Noxious. $\mathrm{OC}, \mathrm{CL}$.

BUTOMACEAE (Flowering-Rush Family)

Butomus umbellatus L. - Greater Flowering-Rush. CH [K]. Non-native.

CABOMBACEAE (Watershield Family)

Brasenia schreberi J.F. Gmel. - Watershield. CH [K]. Native. Rare. Reported in two counties in Kansas, but common in surrounding states.

CACTACEAE (Cactus Family)

Opuntia cespitosa Raf. - Devil's-Tongue. CRW [!, K, G]. Native. OC. This taxon formerly was recognized as Opuntia humifusa (Raf.) Raf. (Majure et al. 2017).

Opuntia macrorhiza Engelm. - Twist-Spine Prickly-Pear. CH [K], CRW [!, K] Native. CL.

CAMPANULACEAE (Bellflower Family)

Campanulastrum americanum (L.) - Small American-Bellflower. $\mathrm{CH}$ $[!, \mathrm{K}]$, CRW [!, K, G]. Native. OP, OC, CL.

Lobelia appendiculata A. DC. - Pale Lobelia. CH [K], CRW [K]. Native. Rare. Reported in five counties in Kansas; not known from Missouri but common in Oklahoma and Arkansas.

Lobelia cardinalis L. - Cardinal-Flower. CH [!, K], CRW [!, K, G]. Native. $\mathrm{OP}, \mathrm{OC}, \mathrm{CL}$.

Lobelia inflata L. - Indian-Tobacco. CH [!, K], CRW [!]. Native. Rare. CL. Crawford County Record (Pryer 1473, Pryer 1603a). Reported in nine counties in Kansas, including this report; common in surrounding states.

Lobelia siphilitica L. - Great Blue Lobelia. CH [K], CRW [!, K, G]. Native. OC.

Lobelia spicata Lam. - Pale-Spike Lobelia. CH [!, K], CRW [!, K, G]. Native. OP, OC, CL.

Triodanis holzingeri McVaugh - Holzinger's Venus'-Looking-Glass. $\mathrm{CH}[\mathrm{K}], \mathrm{CRW}[\mathrm{K}]$. Native. 
Triodanis lamprosperma McVaugh - Prairie Venus'-Looking Glass. CH [K]. Native. Rare. Reported in three counties in Kansas; common in Oklahoma and Arkansas, but rare in Missouri.

Triodanis leptocarpa (Nutt.) Nieuwl. - Slim-Pod Venus'-LookingGlass. CH [!, K]. Native. CL.

Triodanis perfoliata (L.) Nieuwl. subsp. biflora (Ruiz \& Pavón) Lammers - Clasping-Leaf Venus'-Looking-Glass. CH [K], CRW [K, G]. Native.

Triodanis perfoliata (L.) Nieuwl. subsp. perfoliata - Clasping-Leaf Venus'-Looking-Glass. CH [!, K], CRW [!, K, G]. Native. OP, CL.

\section{CANNABACEAE (Hemp Family)}

Celtis laevigata Willd. - Sugar-Berry. CH [!, K], CRW [!, K, G]. Native. $\mathrm{OC}, \mathrm{CL}$.

Celtis occidentalis L. - Common Hackberry. CH [!, K], CRW [!, K, G]. Native. OP, OC, CL.

Celtis tenuifolia Nutt. - Dwarf Hackberry. CH [K], CRW [K, G]. Native. Humulus lupulus L. - Common Hop. CRW [K, G]. Non-native.

\section{CAPRIFOLIACEAE (Honeysuckle Family)}

Lonicera flava Sims - Yellow Honeysuckle. CH [K]. Native. Rare. Reported in four counties in Kansas, but common in surrounding states.

Lonicerajaponica Thunb. - Japanese Honeysuckle. CH [!, K], CRW [!, $\mathrm{K}, \mathrm{G}$. Non-native. $\mathrm{OC}, \mathrm{CL}$.

Lonicera maackii (Rupr.) Maxim. - Amur Honeysuckle. CH [!], CRW [!, G]. Non-native. CL. Cherokee County Record (Pryer 186, Pryer 3746, Pryer 3938, Pryer 3961, Pryer 4005).

Lonicera reticulata Raf. - Grape Honeysuckle. CH. Native. Rare. K. Known from one collection in Cherokee County (W.H. Horr E480 \& R.L. McGregor [KSP020375]); considered extirpated from Bourbon County.

Lonicera sempervirens L. - Trumpet Honeysuckle. CH [K], CRW [!, K, G]. Native. Rare. CL. Reported in four counties in Kansas; common in surrounding areas but considered adventive in Missouri.

Symphoricarpos orbiculatus Moench - Coral-Berry. CH [!, K], CRW [!, $\mathrm{K}, \mathrm{G}]$. Native. OP, OC, CL.

Triosteum angustifolium L. - Yellow-Fruit Horse-Gentian. CH [K]. Native. Rare. Reported from only Cherokee County, Kansas; common in surrounding areas, except for Oklahoma, where the nearest reports are from southern counties.

Triosteum perfoliatum L. - Feverwort. CH [K], CRW [G]. Native.

\section{CARYOPHYLLACEAE (Pink Family)}

Agrostemmagithago L. - Common Corncockle. CRW [G]. Non-native. Arenaria serpyllifolia L. - Thyme-Leaf Sandwort. CH [!, K], CRW [!, K, $\mathrm{G}$. Non-native. Gibson did not recognize varieties of this taxon. Identification of varieties depends on seeds. If the specimen is too young, it is impossible to state which variety you have. Therefore, some of my specimens lack a varietal designation.

Arenaria serpyllifolia L. var. serpyllifolia - Thyme-Leaf Sandwort. CH [!, K], CRW [!, K]. Non-native. OP, OC, CL. State Record.

Arenaria serpyllifolia L. var. tenuior Mert. \& W.D.J. Koch - ThymeLeaf Sandwort. CH [!]. Non-native. OP. State Record. Cherokee County Vouchers (Pryer 4965, Pryer 5125). This taxon has scattered reports. It has been reported throughout Missouri, the Northwestern half of Arkansas, and none from Oklahoma. Reports come from many bordering Missouri counties.

Cerastium brachypetalum Desportes ex Pers. A. Gray - Mouse-Ear Chickweed. CH [!, K], CRW [!]. Non-native. OP, OC, CL. Crawford County Record (Pryer 4078, Pryer 4336, Pryer 4337, Pryer 4537, Pryer 4556, Pryer 4575).

Cerastium brachypodum (Engelm. ex A. Gray) B.L. Rob. - Short-Stalk Mouse-Ear Chickweed. CH [K], CRW [!, K, G]. Native. OC.

Cerastium fontanum Baumg. subsp. vulgare (Hartman) Greuter \& Burdet - Common Mouse-Ear Chickweed. CH [!, K]. CRW [!, K]. Non-native. OP, OC, CL.
Cerastium glomeratum Thuill. - Sticky Mouse-Ear Chickweed. CH [!, $\mathrm{K}], \mathrm{CRW}[!, \mathrm{K}, \mathrm{G}]$. Non-native. OP, OC, CL.

Cerastium nutans Raf. var. nutans - Nodding Mouse-Ear Chickweed. $\mathrm{CH}[\mathrm{K}], \mathrm{CRW}[\mathrm{K}]$. Native.

Cerastium pumilum W. Curtis - European Mouse-Ear Chickweed. CH [!, K]. CRW [!]. Non-native. OP, OC. Crawford County Record (Pryer 4456, Pryer 4458, Pryer 4497, Pryer 4587, Pryer 4765).

Dianthus armeria L. - Deptford Pink. CH [!, K], CRW [!, K, G]. Nonnative. $\mathrm{OP}, \mathrm{OC}, \mathrm{CL}$.

Gypsophila elegans Bieb. - Showy Baby's-Breath. CRW [K]. Non-native.

Holosteum umbellatum L. - Jagged-Chickweed. CRW [!, K]. Nonnative. OC.

Minuartia patula (Michx.) Mattf. - Pitcher's Stitchwort. CH [K]. CRW $[!, \mathrm{K}, \mathrm{G}]$. Native. OP, OC, CL.

Paronychia fastigiata (Raf.) Fernald var. fastigiata - Hairy Forked Nailwort. CH [K], CRW [!, K]. Native. OC.

Sagina decumbens (Elliott) Torr. \& A. Gray subsp. decumbens Trailing Pearlwort. CH [K], CRW [Snow 10775]. Native.

Saponaria officinalis L. - Bouncing-Bett. CH [K], CRW [!, K, G]. Nonnative. $\mathrm{OP}, \mathrm{CL}$.

Scleranthus annuus L. - Annual Knawel. CH [K], CRW [!, K]. Nonnative. OC.

Silene antirrhina L. - Sleepy Catchfly. CH [K], CRW [!, K, G]. Native. $\mathrm{OP}, \mathrm{OC}, \mathrm{CL}$.

Silene regia Sims - Royal Catchfly. $\mathrm{CH}[\mathrm{K}]$. Native. Extirpated. The most recent specimen was found at McGregor Herbarium, University of Kansas, made on July 24, 1995 (K.M. Highfill s.n.).

Silene stellata (L.) W.T. Aiton - Widow's-Frill. CH [!, K], CRW [!, K, G]. Native. OP, OC, $\mathrm{CL}$.

Silene virginica L. - Fire-Pink. CRW [K, G]. Native.

Stellaria media (L.) Vill. - Common Chickweed. CH [!, K], CRW [!, K, G]. Non-native. OP, OC, CL.

Stellaria neglecta Weihe - Greater Chickweed. CH [!], CRW [!]. Nonnative. OP, OC, CL. State Record. Cherokee County vouchers (Pryer 4054, Pryer 4159, Pryer 4196, Pryer 4964). Crawford County vouchers (Pryer 33, Pryer 49, Pryer 64, Pryer 127, Pryer 4069, Pryer 4070, Pryer 4231, Pryer 4232, Pryer 4268, Pryer 4316, Pryer 4373, Pryer 4533, Pryer 4584). Abundant in both counties. This taxon has been reported in few counties nationwide, including one in Arkansas, three in Missouri, and one in Oklahoma. The closest report is from Ottawa County, Oklahoma.

Stellaria pallida (Dumort) Crépin - Lesser Chickweed. CRW [K]. Nonnative. Not reported by Gibson (1963) and present in about 14 counties in Kansas (Kartesz 2019). Vouchers for Crawford county at KSC include Stahl s.n. (KSC cat. no. 18825; det. by McGregor) and Holland 10382 (KSC cat. no. 18808; det. by collector, confirmed by M. Mayfield in 2019).

CELASTRACEAE (Bittersweet Family)

Celastrus orbiculatus Thunb. - Asian Bittersweet. CRW [!]. Non-native. CL. Crawford County Record (Pryer 337, Pryer 3283, Pryer 3458).

Celastrus scandens L. - American Bittersweet. CH [!, K], CRW [!, K, G]. Native. OP, CL.

Elaeodendron fortunei Turcz. - Winter-Creeper. CH [!, K] CRW [!]. Non-native. CL. Crawford County Record (Pryer 2245).

Euonymus alatus (Thunb.) Siebold - Winged Spindletree. CH [K], CRW [!]. Non-native. CL. Crawford County Record (Pryer 6412).

Euonymus atropurpureus Jacq. var. atropurpureus - Eastern Wahoo. $\mathrm{CH}[!, \mathrm{K}], \mathrm{CRW}[!, \mathrm{K}, \mathrm{G}]$. Native. OP, OC, CL. Gibson did not recognize varieties of this taxon.

CERATOPHYLLACEAE (Hornwort Family)

Ceratophyllum demersum L. - Coon's-Tail. CH [!, K], CRW [!, K, G]. Native. OP, OC, CL. 
CISTACEAE (Rock-Rose Family)

Lechea mucronata Raf. - Hairy Pinweed. CH [K]. Native. Rare. Reported in eight counties in Kansas, but common in surrounding states.

Lechea tenuifolia Michx. - Narrow-Leaf Pinweed. CH [!, K], CRW [G]. Native. OP.

CLEOMACEAE (Spider-Flower Family)

Polanisia dodecandra (L.) DC. subsp. trachysperma (Torr. \& A. Gray) Iltis - Red-Whisker Clammyweed. CH [K]. Native.

COLCHICACEAE (Autumn-Crocus Family)

Uvularia grandiflora Sm. - Large-Flower Bellwort. CH [!, K]. Native. Rare. Reported in five counties in Kansas, but common in Missouri and Arkansas; considered rare in the Oklahoma.

COMMELINACEAE (Spiderwort Family)

Commelina communis L. - Asiatic Dayflower. CH [!, K], CRW [!, K, G]. Non-native. $\mathrm{OC}, \mathrm{CL}$.

Commelina diffusa Burm. f. var. diffusa - Climbing Dayflower. CH [!, K]. Native. Rare. OP. CL. Reported in ten counties in Kansas, but common in surrounding states.

Commelina erecta L. - White-Mouth Dayflower. CH [!, K], CRW [!]. Native. OP, OC, CL. Crawford County Record (Pryer 1994, Pryer 5939, Pryer 5956, Pryer 5968)

Commelina virginica L. - Virginia Dayflower. CH [K], CRW [!]. Native. CL. Crawford County Record (Pryer 3240).

Tradescantia bracteata Small - Long-Bract Spiderwort. CH [K], CRW $[!$, G]. Native. OC.

Tradescantia hirsutiflora Bush - Hairy-Flower Spiderwort. CH [K], CRW [G]. Native.

Tradescantia ohiensis Raf. - Bluejacket. CH [!, K], CRW [!, K, G]. Native. $\mathrm{OP}, \mathrm{OC}, \mathrm{CL}$.

CONVOLVULACEAE (Morning-Glory Family)

Calystegia macounii (Greene) Brummitt - Macoun's False Bindweed. CH [!]. Native. Noxious. CL. Cherokee County Record (Pryer 626b).

Calystegia sepium (L.) R. Br. subsp. angulata Brummitt - Hedge False Bindweed. CH [!], CRW [!, G]. Native. Noxious. OC. CL. Cherokee County Record (Pryer 2446). Crawford County Record (Pryer 5754).

Calystegia silvatica (Kit.) Griseb. subsp. fraterniflora (Mack. \& Bush) Brummitt - Short-Stalk False Bindweed. CH [!, K], CRW [!, K, G]. Native. Noxious. OP, CL.

Convolvulus arvensis L. - Field Bindweed. CH [!, K], CRW [!, K, G]. Non-native. Noxious. OP, CL.

Cuscuta compatca Juss. ex Choisy-Compact Dodder. CRW [Holland 6780 (KSP025000)]. State Record. The closest known occurrences are Jasper County, Missouri, and Delaware County, Oklahoma. More common east and (especially) south.

Cuscuta cuspidata Engelm. - Cusp Dodder. CH [!, K], CRW [!, K, G]. Native. Noxious. OP, OC, CL.

Cuscuta glomerata Choisy - Rope Dodder. CH [K], CRW [K, G]. Native. Noxious.

Cuscuta gronovii Willd. ex J.A. Schultes var. gronovii - Scaldweed. $\mathrm{CH}[!, \mathrm{K}], \mathrm{CRW}[!, \mathrm{K}, \mathrm{G}]$. Native. Noxious. OP, OC, CL.

Cuscuta pentagona Engelm. var. pentagona - Bush-Clover Dodder. $\mathrm{CH}$ [!, K], CRW [!, K, G]. Native. Rare. OP, CL. Gibson did not recognize varieties of this taxon. Reported in thirty-nine counties in Kansas, but common in surrounding states. Noxious.

Cuscuta polygonorum Engelm. - Smartweed Dodder. CH [K], CRW [!, K, G]. Native. Noxious. OC.

Ipomoea hederacea Jacq. - Ivy-leaf morning-glory. CH [!, K], CRW [!, $\mathrm{K}, \mathrm{G}]$. Native. Noxious. CL.
Ipomoea lacunosa L. - Whitestar. CH [!, K], CRW [!, K, G]. Native. Noxious. OP, OC.

Ipomoea pandurata (L.) G.F.W. Mey. - Man-of-the-earth. CH [!, K], CRW [!, K]. Native. Noxious. OC, CL.

Ipomoea purpurea (L.) Roth. - Common morning-glory. CH [!, K], CRW [!, K, G]. Non-native. Noxious. CL.

CORNACEAE (Dogwood family)

Cornus drummondii C.A. Mey. - Rough-leaf dogwood. CH [!, K], CH [!, K, G]. Native. OC, CL.

Cornus florida L. - Flowering Dogwood. CH [!, K], CRW [!]. Native. OP, CL. Crawford County Record (Pryer 6422).

Cornus obliqua Raf. - Pale Dogwood. CH [K], CRW [K, G]. Native.

CRASSULACEAE (Stonecrop Family)

Hylotelephium erythrostictum (Miq.) H. Ohba - Garden AnnualStonecrop. $\mathrm{CH}[\mathrm{K}]$. Non-native.

Sedum pulchellum Michx. - Widow's-Cross. CH [!, K], CRW [!, K, G]. Native. $\mathrm{OC}, \mathrm{CL}$.

Sedum sarmentosum Bunge - Stringy Stonecrop. CRW [Snow 10974, G]. Non-native. CL.

CUCURBITACEAE (Cucumber Family)

Citrullus lanatus (Thunb.) Matsumura \& Nakai var. lanatus Watermelon. $\mathrm{CH}$ [K]. Non-native.

Cucurbita melopepo L. subsp. melopepo - Field Pumpkin. CH [K]. Native. Adventive.

Echinocystis lobata (Michx.) Torr. \& A. Gray - Wild Cucumber. CH [K]. Native.

Melothria pendula L. var. pendula - Guadeloupe-Cucumber. CH [!]. Native. Rare. Cherokee County Record (Pryer 669, Pryer 3173). Reported in nine counties in Kansas; This considered rare in Missouri, but common in Oklahoma and Arkansas.

Sicyos angulatus L. - One-Seed Burr-Cucumber. CH [!, K], CRW [!, K, G]. Native. OP, OC, CL.

CYPERACEAE (Sedge family)

Bulbostylis capillaris (L.) Kunth ex C.B. Clarke subsp. capillaris Dense-Tuft Hair Sedge. CH [K]. CRW [K]. Native.

Carex aggregata Mack. - Glomerate Sedge. CH [K]. CRW [!, K]. Native. OC, CL.

Carex albicans Willd. ex Spreng. var. albicans - White-Tinge Sedge. $\mathrm{CH}[!, \mathrm{K}], \mathrm{CRW}[\mathrm{K}]$. Native. OP.

Carex amphibola Steud. - Eastern Narrow-Leaf Sedge. CRW [!]. Native. CL. State Record. Crawford County Voucher (Pryer 1042). This taxon has been reported throughout the south and eastern states and is common in the 4-state region. The closes reports are from Jasper and Vernon counties, Missouri.

Carex annectens (Bickn.) Bickn. - Yellow-Fruit Sedge. CH [!, K], CRW $[!, \mathrm{K}, \mathrm{G}]$. Native. OP, CL.

Carex arkansana (Bailey) Bailey - Arkansas Sedge. CH [K]. Native. Rare. Reported in ten counties in Kanasas and is rare in surrounding areas.

Carex austrina Mack. - Southern Sedge. CH [K]. Native.

Carex bicknellii Britton - Bicknell's Sedge. CH [!, K], CRW [!, K, G]. Native. OP.

Carex blanda Dewey - Eastern Woodland Sedge. CH [!, K], CRW [!, $\mathrm{K}, \mathrm{G}]$. Native. OC. CL.

Carex brevior (Dewey) Mack. - Short-Beak Sedge. CH [!, K], CRW [!, $\mathrm{K}, \mathrm{G}]$. Native. $\mathrm{CL}$.

Carex bushii Mack. - Bush's sedge. CH [!, K], CRW [!, K, G]. Native. $\mathrm{OP}, \mathrm{OC}, \mathrm{CL}$.

Carex caroliniana Schwein. - Carolina sedge. CH [!, K]. Native. Rare. $\mathrm{CL}$. Reported in three counties in Kansas, but common in surrounding areas.

Carex cephalophora Muhl. exWilld. - Oval-leaf sedge. CH [!, K], CRW 
[!]. Native. Rare. OP. Reported in seventeen counties in Kansas; rare in Oklahoma but common in both Missouri and Arkansas.

Carex conjuncta Boott - Soft fox sedge. CH [K]. Native. Rare. Reported in eleven counties in Kansas and common in Missouri, but considered rare in Arkansas and known from only two counties in Oklahoma.

Carex corrugata Fernald - Prune-fruit sedge. CH [!, K], CRW [!]. Native. CL. Crawford County Record (Pryer 567, Pryer 568, Pryer 1297).

Carex crus-corvi Shuttlw. ex Kunze - Raven-foot sedge. CH [!, K], CRW $[!, K, G]$. Native. Rare. CL. Reported in ten counties in Kansas but common in surrounding states.

Carex davisii Schwien. \& Torr. - Davis' sedge. CH [!, K], CRW [!, K, G]. Native. $\mathrm{OC}, \mathrm{CL}$.

Carex emoryi Dewey - Emory's Sedge. CH [K]. Native.

Carex festucacea Schukr. ex Willd. - Fescue sedge. CH [K], CRW [!, K, G]. Native. Rare. CL. Reported in nine counties in Kansas but common in surrounding states.

Carex fissa Mack. var. fissa-Hammock Sedge. CH [K]. Native. Rare. Reported in five counties in Kansas; considered rare in surrounding states, though relatively common in surrounding areas.

Carex flaccosperma Dewey-Thin-Fruit Sedge. CRW [K]. Native. Rare. Reported in two counties in Kansas; considered rare Missouri but common in Oklahoma and Arkansas.

Carex frankii Kunth - Frank's sedge. CH [!, K], CRW [!, K, G]. Native. $\mathrm{OP}, \mathrm{CL}$.

Carex granularis Muhl. ex Willd. - Limestone-Meadow Sedge. CRW [!, K, G]. Native. OC, CL.

Carex gravida Bailey - Heavy Sedge. CH [!, K], CRW [!, K, G]. Native. $\mathrm{OP}, \mathrm{OC}, \mathrm{CL}$.

Carex grayi Carey - Gray's Sedge. CH [!, K]. Native. OP, CL.

Carex grisea Wahlenb. - Inflated Narrow-Leaf Sedge. CH [!, K], CRW $[!, \mathrm{K}, \mathrm{G}]$. Native. OP, OC, CL.

Carex hirsutella Mack. - Fuzzy-Wuzzy Sedge. CH [!, K], CRW [!, G]. Native. Rare. OP, CL. Reported in eleven counties in Kansas but common in surrounding states.

Carex hyalinolepis Steud. - Shoreline Sedge. CH [K], CRW [K, G]. Native.

Carex inops subsp. heliophila (Mack.) Crins - Long-Stolon Sedge. CRW [K]. Native.

Carex jamesii Schwein. - James' Sedge. CRW [!, K]. Native. OC, CL.

Carex leavenworthii Dewey - Leavenworth's Sedge. CH [!, K], CRW $[!, \mathrm{K}, \mathrm{G}]$. Native. OP, CL.

Carex lupulina Muhl. ex Willd. - Hop Sedge. CH [!, K], CRW [!, K, G]. Native. CL.

Carex meadii Dewey-Mead's Sedge. CH [K], CRW [!, K, G]. Native. OC. Carex mesochorea Mack. - Midland Sedge. CH [!], CRW [!, K]. Native. Rare. OP, CL. Cherokee County Record (Pryer 165, Pryer 1207). Reported in ten counties in Kansas, including this report; considered rare in Arkansas and common in Missouri, but not reported for Oklahoma.

Carex microdonta Torr. \& Hook. - Little-Tooth Sedge. CH [!], CRW $[!, \mathrm{K}]$. Native. CL.

Carex missouriensis P. Rothr. \& Reznicek - Missouri Sedge. CRW [K]. Native.

Carex molesta Mack. ex Bright - Troublesome Sedge. CH [!, K], CRW [K]. Native. OP, CL.

Carex muehlenbergii Schkuhr ex Willd. var. enervis Boott Muhlenberg's Sedge. CH [!, K], CRW [!]. Native. Rare. OC, CL. Crawford County Record (Pryer 302, Pryer 1159, Pryer 4467). Reported in fourteen counties in Kansas, including this report and common in surrounding states.

Carex muehlenbergii Schkuhr ex Willd. var. muehlenbergii Muhlenberg's Sedge. CH [!], CRW [!]. Native. OP, OC. Cherokee
County Record (Pryer 414, Pryer 412). Crawford County Record (Pryer 4650).

Carex normalis Mack. - Greater Straw Sedge. CH [K], CRW [!]. Native. OC. Crawford County Record (Pryer 723, Pryer 1057, Pryer 4657, Pryer 4846).

Carex oklahomensis Mack. - Oklahoma Sedge. CH [K], CRW [K, G]. Native. Rare. Reported in four counties in Kansas, but common in surrounding areas; considered rare in Oklahoma but common in Missouria and Arkansas.

Carex oligocarpa Schkuhr ex Willd. - Richwoods Sedge. CRW [!, K]. Native. OC, CL.

Carex opaca (F.J. Herm.) P. Rothr. \& Reznicek - CH [!, K], CRW [!, K]. Native. CL.

Carex pellita Muhl. ex Willd. - Woolly Sedge. CRW [!, G]. Native. CL.

Carex radiata (Wahlenb.) Small - Eastern Star Sedge. CH [K], CRW [K, G]. Native. Rare. Reported in eight counties in Kansas, but common in Missouri; considered rare in Arkansas.

Carex retroflexa Muhl. ex Willd. - Reflexed Sedge. CH [K], CRW [K]. Native. Rare. Reported in seven counties in Kansas, but common in surrounding states.

Carex rosea Schkuhr ex Willd. - Rosy Sedge. CH [K], CRW [!]. Native. Rare. CL. Crawford County Record (Pryer 616). Reported in nine counties in Kansas, including this report; common in Missouri and Arkansas but rare in Oklahoma.

Carex scoparia Schkuhr ex Willd. - Pointed Broom Sedge. CH [K], CRW [!, K]. Native. CL.

Carex shortiana Dewey - Short's Sedge. CH [!, K], CRW [!, K, G]. Native. OC, CL.

Carex sparganioides Muhl. ex Willd. - Burr-Reed Sedge. CH [!], CRW [!, K]. Native. OP, CL. Cherokee County Record (Pryer 420, Pryer 508, Pryer 525, Pryer 949, Pryer 1206, Pryer 1208, Pryer 1210a, Pryer 1218, Pryer 5088)

Carex squarrosa L. - Squarrose Sedge. CH [K], CRW [K, G]. Native. Rare. Reported in three counties in Kansas, but common in surrounding areas.

Carex triangularis Boeckeler - Eastern Fox Sedge. CH [K]. Native. Rare. Reported in only Cherokee County, Kansas. This taxon is common in Oklahoma and Arkansas, but considered rare in Missouri.

Carex tribuloides Wahlenb. var. sangamonensis Clokey - Blunt Broom Sedge. CH [!, K], CRW [!]. Native. CL. Crawford County Record (Pryer 994, Pryer 1459, Pryer 1460, Pryer 1461, Pryer 2233, Pryer 2234).

Carex umbellata Schkuhr ex Willd. - Parasol Sedge. CH [K], CRW [K]. Native.

Carex vulpinoidea Michx. - Common Fox Sedge. CH [!, K], CRW [K, G]. Native. CL.

Cyperus acuminatus Torr. \& Hook. ex Torr. - Taper-Tip Flat Sedge. CH $[!, \mathrm{K}]$, CRW [!, K, G]. Native. OP, CL.

Cyperus echinatus (L.) Wood - Globe Flat Sedge. CH [!, K], CRW [!, $\mathrm{K}, \mathrm{G}]$. Native. OP, CL.

Cyperus erythrorhizos Muhl. - Red-Root Flat Sedge. CH [!, K], CR [!]. Native. OP, OC, CL. Crawford County Record (Pryer 2758, Pryer 2759, Pryer 2808, Pryer 3331, Pryer 3453, Pryer 3455).

Cyperus esculentus L. var. leptostachyus Boeckeler - Chufa CH [!, K], CRW [!, K]. Naitve. OP, CL. Gibson did not recognize varieties of this taxon.

Cyperus hortensis (Salzm. \& Steud.) Dorr - Low Spike Sedge. CH [K], CRW [!]. Native. Rare. CL. Crawford County Record (Pryer 3561, Pryer 5688). In Kansas known from four eastern counties; common in Missouri, Arkansas and eastern Oklahoma.

Cyperus lupulinus (Spreng.) Marcks subsp. lupulinus - Great Plains Flat Sedge. CH [!, K], CRW [!, K]. Native. OP, OC, CL.

Cyperus odoratus L. - Rusty Flat Sedge. CH [!, K], CRW [!, K, G]. Native. OC, CL. 
Cyperus pseudovegetus Steud. - Marsh Flat Sedge. CH [!, K], CRW [!, $\mathrm{K}$ ]. Native. Rare. OP, CL. Reported in fifteen counties in Kansas but common in surrounding states.

Cyperus squarrosus L. - Awned Flat Sedge. CH [K], CRW [!, K, G]. Native. OC, CL.

Cyperus strigosus L. - Straw-Color Flat Sedge. CH [!, K], CRW [!, K, G]. Native. OP, OC, CL.

Eleocharis acicularis (L.) Roemer \& J.A. Schultes - Needle Spike-Rush. $\mathrm{CH}[!, \mathrm{K}], \mathrm{CRW}[!, \mathrm{K}, \mathrm{G}]$. Native. CL.

Eleocharis compressa Sullivant - Flat-Stem Spike-Rush. CH [!], CRW [!, G]. Naitve. CL.

Eleocharis engelmannii Steud. - Engelmann's Spike-Rush. CH [!, K], CRW [!, K]. Native. OC, CL.

Eleocharis lanceolata Fernald - Dagger-Leaf Spike-Rush. CH [K], CRW [!]. Natie. Rare. CL. Crawford County Record (Pryer 5494). Reported in six counties in Kansas, including this report; common in Oklahoma and Arkansas but considered rare in Missouri.

Eleocharis obtusa (Willd.) J.A. Schultes - Blunt Spike-Rush. CH [!, K], CRW [!, K, G]. Native. OP, OC, CL.

Eleocharis palustris (L.) Roemer \& J.A. Schultes - Common SpikeRush. CH [!, K], CRW [!, K, G]. Native. OC, CL.

Eleocharis parvula (Roemer \& J.A. Schultes) Link ex Bluff, Nees \& Schauer - Little-Head Spike-Rush. CRW [!]. Native. OC, CL. Crawford County Record (Pryer 2123, Pryer 5492, Pryer 5692).

Eleocharis tenuis (Willd.) J.A. Schultes var. verrucosa (Svens.) Svens. Slender Spike-Rush. CH [K], CRW [!]. Native. Rare. OC. Crawford County Record (Pryer 2788). Reported in twelve counties in Kansas, but common in surrounding states.

Eleocharis wolfii (A. Gray) A. Gray ex Britton - Wolf's Spike-Rush. $\mathrm{CH}[\mathrm{K}], \mathrm{CRW}[\mathrm{K}]$. Native. Rare. Reported in eight counties in Kansas and common in surrounding areas; considered rare in Oklahoma and Arkansas but common in Missouri.

Fimbristylis annua (All.) Roemer \& J.A. Schultes - Annual Fimbry. CH [K], CRW [K]. Native. Rare.

Fimbristylis autumnalis (L.) Roemer \& J.A. Schultes - Slender Fimbry. CRW [!, K]. Native. Rare. CL. Reported in thirteen counties in Kansas but common in surrounding states.

Fimbristylis puberula (Michx.) Vahl var. puberula - Hairy Fimbry. $\mathrm{CH}$ [!, K], CRW [!, K, G]. Native. OC, CL.

Fimbristylis vahlii (Lam.) Link - Vahl's Fimbry. CH [K], CRW [K]. Native. Rare. Reported in nine counties in Kansas but common in surrounding states.

Isolepis carinata Hook. \& Arn. ex Torr. - Keeled Lateral-Bulrush. CH [!, K], CRW [K]. Native. OP.

Kyllinga pumila Michx. - Low Spike Sedge. CH [K], CRW [!]. Native. Rare. CL. Crawford County Record (Pryer 3561, Pryer 5688).

Rhynchospora harveyi W. Boott var. harveyi - Harvey's Beak Sedge. $\mathrm{CH}[\mathrm{K}], \mathrm{CRW}[\mathrm{K}]$. Native. Rare. Reported in eight counties in Kansas, but common in the surrounding areas; considered rare in Missouri but common in Oklahoma and Arkansas.

Rhynchospora recognita (Gale) Kral - Coarse Globe Beak Sedge. CH [!, K]. CRW [!]. Native. Rare. OP, OC, CL. Crawford County Record (Pryer 5289, Pryer 5299). Reported in five counties in Kansas, including this report, but common in surrounding areas.

Schoenoplectus deltarum (Schuyler) Soják - Delta Club-Rush. CH [K], CRW [K]. Native. Rare. Reported in four counties in Kansas, but has few reports nationwide and is considered rare wherever it is reported.

Schoenoplectus pungens (Vahl) Palla var. longispicatus (Britton) S.G. Sm. - Three-Square. $\mathrm{CH}[\mathrm{K}]$. Native.

Scirpus atrovirens Willd. - Dark-Green Bulrush. CH [K], CRW [!, K, G]. Native. OC.

Scirpus cyperinus (L.) Kunth - Cottongrass Bulrush. CRW [!]. Native. $\mathrm{CL}$.
Scirpus georgianus Harper - Georgia Bulrush. CH [!, K], CRW [!, K]. Native. $\mathrm{OP}, \mathrm{CL}$.

Scirpus pendulus Muhl. - Rufous Bulrush. CH [!, K], CRW [!, K, G]. Native. OP, OC, CL.

Scleria ciliata Michx. - Fringed Nut-Rush. CH [K]. Native. Rare. Reported in 9 counties in Kansas, but common in surrounding states.

Scleria pauciflora Muhl. ex Willd. var. caroliniana (Willd.) Wood - FewFlower Nut-Rush. CH [K]. Native. Rare. Reported in Cherokee County, Kansas, but common in the surrounding areas; considered rare in Arkansas.

Scleria pauciflora Muhl. ex Willd. var. pauciflora - Few-Flower NutRush. CH [K]. Native. Rare. Reported in eight counties in Kansas, but common in surrounding areas.

Scleria triglomerata Michx. - Whip Nut-Rush. CH [!, K], CRW [!, K]. Native. OC, CL.

\section{DIOSCOREACEAE (Yam Family)}

Dioscorea polystachya Turcz. - Chinese Yam. CH [!, K], CRW [G]. Non-native. OP.

Dioscorea villosa L. - Wild Yam. CH [!, K], CRW [K]. Native. OC.

DIPSACACEAE (Teasel Family)

Dipsacus fullonum L. - Fuller's Teasel. CRW [!, K]. Non-native. OC.

Dipsacus laciniatus L. - Cut-Leaf Teasel. CH [!], CRW [!, K]. Non-native. OC, CL. Cherokee County Record (Pryer 3779).

DROSERACEAE (Sundew Family)

Drosera brevifolia Pursh - Dwarf Sundew. CH [K]. Native. Rare. Reported only from Cherokee County, Kansas and rare in surrounding areas; considered rare in Oklahoma, common in Arkansas, and not yet reported for Missouri.

EBENACEAE (Ebony Family)

Diospyros virginiana L. - Common Persimmon. CH [!, K], CRW [!, K, G]. Native. OP, OC, CL.

ELAEAGNACEAE (Oleaster Family)

Elaeagnus angustifolia L. - Russian-Olive. CH [K], CRW [G]. Non-native.

Elaeagnus umbellata Thunb. - Autumn-Olive. CH [!], CRW [!, K]. Non-native. CL. Cherokee County Record (Pryer 183, Pryer 1655, Pryer 3736, Pryer 3865, Pryer 3977, Pryer 4004, Pryer 4010, Pryer 4012, Pryer 4950; Snow \& Sanders 11,138).

EQUISETACEAE (Horsetail Family)

Equisetum hyemale L. var. affine (Engelm.) A.A. Eaton - Tall ScouringRush. CH [!, K]. Native. OP.

Equisetum $\times$ ferrissii Clute (pro sp.) - $\mathrm{CH}[\mathrm{K}]$. Native.

ERICACEAE (Heath Family)

Hypopitys monotropa Crantz - Yellow Bird's-Nest. CH [K]. Native. Rare. Reported from two counties in Kansas; common in Missouri and Arkansas but rare in Oklahoma.

Vaccinium arboreum Marshall - Tree Sparkle-Berry. CH [K]. Native. Rare. Only reported in Cherokee County, Kansas, but common in surrounding states.

Vaccinium pallidum Aiton - Early Lowbush Blueberry. CH [!, K]. Native. Rare. Only reported in Cherokee County, Kansas, but common in surrounding states.

Vaccinium stamineum L. - Deerberry. CH [K]. Native. Rare. Only reported in Cherokee County, Kansas, but common in surrounding states.

EUPHORBIACEAE (Spurge Family)

Acalypha gracilens A. Gray - Slender Three-Seed-Mercury. CH [!], CRW [!]. Native. Rare. OP, OC, CL. Cherokee County Record (Pryer 3109, Pryer 3400, Pryer 5632, Pryer 5819). Crawford County Record (Pryer 2147, Pryer 2544, Pryer 6041, Pryer 6258). 
Only reported in Cherokee County, Kansas, but common in surrounding states.

Acalypha monococca (Engelm. ex A. Gray) L. Mill. \& Gandhi - SingleSeed Three-Seed-Mercury. CH [!, K], CRW [!, K, G]. Ntive. OP, $\mathrm{OC}, \mathrm{CL}$.

Acalypha ostryifolia Riddell - Pineland Three-Seed-Mercury. CH [!, K], CRW [!, K]. Native. OP, OC.

Acalypha rhomboidea Raf. - Common Three-Seed-Mercury. CH [!, $\mathrm{K}]$, CRW [!, K]. Native. OP, OC, CL.

Acalypha virginica L. - Virginia Three-Seed-Mercury. CH [!, K], CRW $[!, \mathrm{K}, \mathrm{G}]$. Native. OP, OC, CL.

Croton capitatus Michx. - Hogwort. CH [!, K], CRW [!, K, G]. Native. $\mathrm{OP}, \mathrm{OC}, \mathrm{CL}$.

Croton glandulosus L. var. septentrionalis Muell.-Arg. - VenteConmigo. CH [!, K], CRW [!, K, G]. Native. OP, CL. Gibson did not recognize varities of this taxon.

Croton michauxii G.L. Webster var. ellipticus (Willd.) B.W. van Ee \& P.E. Berry - Narrow-Leaf Rushfoil. CH [!, K]. Native. OP.

Croton monanthogynus Michx. - Prairie-Tea. CH [!, K], CRW [!, K, G]. Native. $\mathrm{OP}, \mathrm{OC}, \mathrm{CL}$.

Euphorbia corollata L. - Flowering Spurge. CH [!, K], CRW [!, K, G]. Native. OP, OC, CL.

Euphorbia cyathophora Murr. - Fire-on-the-Mountain. CH [!, K], CRW [K]. Native. OP.

Euphorbia davidii Subils - David's Spurge. Ch [K], CRW [!, K]. Nonnative. $\mathrm{OP}, \mathrm{CL}$.

Euphorbia dentata Michx. var. dentata - Toothed Spurge. CH [!, K], CRW [!, K]. Native. OP, OC, CL.

Euphorbia humistrata Engelm. ex A. Gray-Spreading Sandmat. $\mathrm{CH}$ [!, K]. CRW [G]. Native. OP, CL.

Euphorbia glyptosperma Engelm. - Rib-Seed Sandmat. CH [!]. Native. CL. Cherokee County Record (Pryer 4029).

Euphorbia maculata L. - Spotted Sandmat. CH [!, K], CRW [!, K, G]. Native. OP, OC, CL.

Euphorbia marginata Pursh - Snow-on-the-Mountain. CH [K], CRW [K]. Native.

Euphorbia missurica Raf. - Prairie Sandmat. $\mathrm{CH}[\mathrm{K}], \mathrm{CRW}[\mathrm{K}, \mathrm{G}]$. Native.

Euphorbia nutans Lag. - Eyebane. CH [!, K], CRW [!, K, G]. Native. $\mathrm{OP}, \mathrm{OC}, \mathrm{CL}$.

Euphorbia prostrata Aiton - Prostrate Sandmat. CR [!, K]. Native. CL.

Euphorbia pubentissima Michx. - False Flowering Spurge. CH [K]. Native.

Euphorbia serpens Kunth - Matted Sandmat. CH [K], CRW [!, K, G]. Native. OC, CL.

Euphorbia spathulata Lam. - Warty Spurge. CH [K], CRW [!, G]. Native. OC.

Euphorbia stictospora Engelm. - Slim-Seed Sandmat. CH [!, K]. Native. OP, CL.

Tragia betonicifolia Nutt. - Betony-Leaf Noseburn. CH [!, K], CRW [!, $\mathrm{K}, \mathrm{G}$. Native. $\mathrm{OC}, \mathrm{CL}$.

FABACEAE (Pea Family)

Acmispon americanus (Nutt.) Rydb. var. americanus - American Deerweed. CH [K], CRW [!, K, G]. Native. CL.

Albizia julibrissin Durazz. - Silktree. CH [!, K], CRW [!, K]. Native. CL. Amorpha canescens Pursh - Leadplant. CH [!, K], CRW [!, K, G]. Native. OP, OC, CL.

Amorpha fruticosa L. - False Indigo-Bush. CH [!, K], CRW [!, K, G]. Native. OC, CL.

Amphicarpaea bracteata (L.) Fernald var. bracteata - American Hog-Peanut. CH [!], CRW [!]. Native. OP. Cherokee County Record (Pryer 1747, Pryer 6294). Crawford County Record (Pryer 6157, Pryer 6163).

Apios americana Medik. - Groundnut. CH [K], CRW [K, G]. Native.
Astragalus canadensis L. var. canadensis - Canadian Milk-Vetch. CH [K], CRW [!, K]. Native. CL.

Astragalus crassicarpus Nutt. var. crassicarpus - Ground-Plum. CH [K], CRW [!, K, G]. Native. OC.

Astragalus crassicarpus Nutt. var. trichocalyx (Nutt.) Barneby Ground-Plum. CH [K], CRW [!]. Native. OC. Crawford County Record (Pryer 1986, Pryer 4808).

Astragalus distortus Torr. \& A. Gray var. distortus - Ozark Milk-Vetch. $\mathrm{CH}[\mathrm{K}], \mathrm{CRW}[\mathrm{K}]$. Native.

Baptisia alba (L.) Vent. var. macrophylla (Larisey) Isely - White Wild Indigo. CH [!, K], CRW [!, K, G]. Native. OP, CL.

Baptisia australis (L.) R. Br. var. minor (Lehm.) Fernald - Blue Wild Indigo. CH [K], CRW [!, K, G]. Native. OC.

Baptisia bracteata Muhl. ex Elliott var. leucophaea (Nutt.) Kartesz \& Gandhi - Long-Bract Wild Indigo. CH [!, K], CRW [!, K, G]. Native. OP, OC, CL.

Baptisia ×bicolor Greenm. \& Larisey - CR [!]. Native. OC. Crawford County Record (Pryer 4470, Pryer 4473, Pryer 4505).

Cercis canadensis L. var. canadensis - Redbud. CH [!, K], CRW [!, K, G]. Native. OP, OC, CL.

Chamaecrista fasciculata (Michx.) Greene var. fasciculata Sleepingplant. CH [!, K], CRW [!, K, G]. Native. OP, OC, CL.

Chamaecrista nictitans (L.) Moench var. nictitans - Partridge-Pea. $\mathrm{CH}[!, \mathrm{K}]$. Native. OP, OC.

Clitoria mariana L. - Atlantic Pigeonwings. $\mathrm{CH}[\mathrm{K}]$. Native. Extirpated.

Colutea arborescens L. - Bladder-Senna. CRW [!, K, G]. Non-native. CL.

Crotalaria sagittalis L. - Arrow-Head Rattlebox. CH [!, K], CRW [!, K, $\mathrm{G}]$. Native. OP, OC, CL.

Dalea candida Michx. ex Willd. var. candida - White Prairie-Clover. $\mathrm{CH}[!, \mathrm{K}], \mathrm{CRW}[!, \mathrm{K}, \mathrm{G}]$. Native. OC, CL.

Dalea leporina (Aiton) Bullock - Fox-Tail Prairie-Clover. CRW [K]. Native.

Dalea purpurea Vent. - Violet Prairie-Clover. CH [!, K], CRW [!, K, G]. Native. OC, CL.

Desmanthus illinoensis (Michx.) MacMill. ex B.L. Rob. \& Fernald Prairie Bundle-Flower. CH [!, K], CRW [!, K, G]. Native. OC, CL.

Desmodium canadense (L.) DC. - Showy Tick-Trefoil. CH [K], CRW [K, G]. Native.

Desmodium canescens (L.) DC. - Hoary Tick-Trefoil. CH [K], CRW [K, G]. Native.

Desmodium ciliare (Muhl. ex Willd.) DC. - Hairy Small-Leaf TickTrefoil. CH [K], CRW [!, K, G]. Native. Rare. CL. Reported in ten counties in Kansas, but common in surrounding states.

Desmodium cuspidatum (Muhl. ex Willd.) DC. ex Loud. - Large-Bract Tick-Trefoil. CH [!, K], CRW [K, G]. Native. OP.

Desmodium glabellum (Michx.) DC. - Dillenius' Tick-Trefoil. CH [!, K], CRW [!, G]. Native. OC, CL. Crawford County Record. (Pryer 3585, Pryer 6049).

Desmodium illinoense A. Gray - Illinois Tick-Trefoil. CH [K], CRW [K]. Native.

Desmodium marilandicum (L.) DC. var. ciliare (Muhl. ex Willd.) $\mathrm{H}$. Ohashi - Hairy Small-Leaf Tick-Trefoil. CH [K], CRW [!, K, G]. Native. Rare. CL. In Kansas known from ten eastern counties; common east and south.

Desmodium marilandicum (L.) DC. var. lancifolium (Fern. \& B.G. Schub.) H. Ohashi-Stiff Tick-Trefoil. CH [!, K], CRW [!, G]. Native. Rare. OC, CL. Crawford County Record. (Pryer 3661). In Kansas known from six southeastern counties; more common east and south.

Desmodium marilandicum (L.) DC. var. marilandicum - Smooth Small-Leaf Tick-Trefoil. CH [!, K], CRW [!, K]. Native. OP, CL.

Desmodium obtusum (Muhl. ex Willd.) DC. - Stiff Tick-Trefoil. CH [!, $\mathrm{K}], \mathrm{CRW}[!, \mathrm{G}]$. Native. OC, CL. 
Desmodium paniculatum (L.) DC. - Panicled-Leaf Tick-Trefoil. CH [!, $\mathrm{K}], \mathrm{CRW}[!, \mathrm{K}, \mathrm{G}]$. Native. OP, OC, CL.

Desmodium perplexum Schub. - Perplexed Tick-Trefoil. CH [!, K], CRW [!, K, G]. Native. OP, CL.

Desmodium rotundifolium DC. - Prostrate Tick-Trefoil. CH [K]. Native. Rare. Only reported in Cherokee County, Kansas, but common in surrounding states.

Desmodium sessilifolium (Torr.) Torr. \& A. Gray - Sessile-Leaf TickTrefoil. CH [!, K], CRW [!, K, G]. Native. CL.

Desmodium sp. - Species unidentifiable; flowers much larger than other species of Desmodium

Desmodium viridiflorum (L.) DC. - Velvet-Leaf-Tick-Trefoil. CH [!]. Native. CL. State Record. Cherokee County Voucher (Pryer 5888). This taxon has been reported throughout the southeastern United States with scattered reports in Oklahoma and Missouri. Considered rare in Missouri, with the closest reports from Barry County, Missouri and Delaware County, Oklahoma.

Galactia regularis (L.) B.S.P. - Eastern Milk-Pea. CH [K], CRW [!, K]. Native. Rare. CL. Reported in two counties in Kansas, but common in surrounding states.

Galactia volubilis (L.) Britton var. volubilis - Downy Milk-Pea. CH [K]. Native.

Gleditsia triacanthos L. - Honey-Locust. CH [!, K], CRW [!, K, G]. Native. OP, OC, CL.

Glycyrrhiza lepidota Pursh - American Licorice. CRW [K, G]. Native.

Gymnocladus dioicus (L.) K. Koch - Kentucky Coffeetree. CH [K], CRW [!, K, G]. Native. OC.

Hylodesmum glutinosum (Willd.) H. Ohashi \& R.R. Mill - Pointed-Leaf Tick-Clover. CH [!, K], CRW [!, K, G]. Native. OP, OC, CL.

Hylodesmum nudiflorum (L.) H. Ohashi \& R.R. Mill - Naked-Flower Tick-Clover. CH [K]. Native. Rare. Only reported in Cherokee County, Kansas, but common in surrounding states.

Hylodesmum pauciflorum (Nutt.) H. Ohashi \& R.R. Mill - Few-Flower Tick-Clover. CH [!, K]. Native. Rare. OP. Reported in two counties in Kansas, but common in Missouri and Arkansas; rare in Oklahoma.

Kummerowia stipulacea (Maxim.) Makino - Korean-Clover. CH [!, K], CRW [!, K, G]. Non-native. OP, OC, CL.

Kummerowia striata (Thunb.) Schindl. - Japanese-Clover. CH [K], CRW [!, K, G]. Non-native. OC, CL.

Ladeania lanceolata (Pursh) A.N. Egan \& Reveal - Wild Lemonweed. $\mathrm{CH}[\mathrm{K}]$. Native.

Lathyrus latifolius L. - Everlasting-Pea. CH [K]. Non-native.

Lathyrus pusillus Elliott - Tiny Vetchling. $\mathrm{CH}[\mathrm{K}], \mathrm{CRW}[\mathrm{K}]$. Native. Rare. Reported in six counties in Kansas; common in Oklahoma but rare in Arkansas and extirpated in Missouri.

Lathyrus venosus Muhl. ex Willd. - Veining Vetchling. CH [!]. Native. OP. State Record (Pryer 1195 [KSP024512]). The nearest records are from Benton County, Arkansas, and Barry County, Missouri. In our general region the species is native mostly to the Missouri Ozarks (where it is rare) and the Ouachita Mountains in Arkansas and Oklahoma; widespread but somewhat sporadic in eastern North America.

Lespedeza capitata Michx. - Round-Head Bush-Clover. CH [K], CRW [K, G]. Native.

Lespedeza cuneata (Dum.-Cours.) G. Don-Chinese Bush-Clover. CH [!, K], CRW [!, K, G]. Non-native. Noxious. OP, CL.

Lespedeza frutescens (L.) Hornem. - Shrubby Bush-Clover. CH [!]. Native. OP. State Record (Pryer 1713, Pryer 2947). A number of specimens collected throughout the years are deposited in the T.M. Sperry Herbarium. Common throughout the eastern United States, including the 4-state region. It has been reported in most all bordering Missouri counties.

Lespedeza hirta (L.) Hornem. subsp. hirta - Hairy Bush-Clover. CH [!, K]. Native. OP.
Lespedeza procumbens Michx. - Trailing Bush-Clover. CH [K]. Native. Rare. Reported in seven counties in Kansas, but common in surrounding states.

Lespedeza repens (L.) W.P.C. Barton - Creeping Bush-Clover. CH [!, K], CRW [!, G]. Native. OP, OC. Crawford County Record (Pryer 2760, Pryer 5767).

Lespedeza $\times$ simulata Mack. \& Bush (pro sp.) - CRW [!, K]. Native. OC. Lespedeza stuevei Nutt. - Tall Bush-Clover. $\mathrm{CH}[\mathrm{K}], \mathrm{CRW}[\mathrm{K}, \mathrm{G}]$. Native. Lespedeza violacea (L.) Pers. - Violet Bush-Clover. CH [!, K], CRW [!, $\mathrm{K}, \mathrm{G}]$. Native. OP, OC.

Lespedeza virginica (L.) Britton - Slender Bush-Clover. CH [!, K], CRW $[!, \mathrm{K}, \mathrm{G}]$. Native. OP, CL.

Lotus corniculatus L. - Garden Bird's-Foot-Trefoil. CH [!], CRW [!]. Nonnative. CL. Cherokee County Record (Pryer 1628, Pryer 4975). Crawford County Record (Pryer 582, Pryer 2156, Pryer 2157).

Lotus tenuis Waldst. \& Kit. ex Willd. - Narrow-Leaf Bird's-Foot-Trefoil. CRW [!]. Non-native. CL. Crawford County Record (Pryer 3898).

Medicago lupulina L. - Black Medick. CH [!, K], CRW [!, K, G]. Nonnative. $\mathrm{OP}, \mathrm{OC}, \mathrm{CL}$.

Medicago minima (L.) L. ex Bartalini - Burr Medick. CH [K], CRW [K]. Non-native.

Medicago sativa L. subsp. sativa - Alfalfa. CH [!, K], CRW [!, K, G] Nonnative. $\mathrm{CL}$. Gibson did not recognize subspecies of this taxon.

Melilotus officinalis (L.) Lam. - Yellow Sweet-Clover. CH [!, K], CRW $[!, \mathrm{K}, \mathrm{G}]$. Non-native. OC, CL.

Mimosa nuttallii (DC.) B.L. Turner - Nuttall's Mimosa. CH [!, K], CRW $[!, \mathrm{K}, \mathrm{G}]$. Native. OP, OC, CL.

Orbexilum pedunculatum (P. Mill.) Rydb. var. pedunculatum Sampson's-Snakeroot. CH [!, K], CRW [!, K, G]. Native. OP, CL.

Pediomelum esculentum (Pursh) Rydb. - Large Indian-Breadroot. CH [!, K], CRW [!, K]. Native. OC.

Pediomelum tenuiflorum (Pursh) A.N. Egan - Slender-Flower IndianBreadroot. CH [!, K], CRW [!, K]. Native. OP, OC, CL.

Pueraria montana (Lour.) Merr. var. lobata (Willd.) Maesen \& S. Almeida - Kudzu. CH [K], CRW [!, K]. Non-native. Noxious. OC.

Robinia hispida L. var. hispida - Bristly Locust. CRW [!]. Native. Adventive. OC. Crawford County Record (Pryer 6426)

Robinia pseudoacacia L. - Black Locust. CH [!, K], CRW [!, K, G]. Native. Adventive. CL.

Securigera varia (L.) Lassen - Purple Crown-Vetch. CH [!, K], CRW [!, K]. Non-native. $\mathrm{OC}, \mathrm{CL}$.

Senna marilandica (L.) Link - Maryland Wild Sensitive-Plant. CH [!, $\mathrm{K}], \mathrm{CRW}[!, \mathrm{K}, \mathrm{G}]$. Native. OP, OC, CL.

Sesbania herbacea (P. Mill.) McVaugh - Peatree. CH [!]. Native. CL. Cherokee County Record (Pryer 5812).

Strophostyles helvola (L.) Elliott - Trailing Fuzzy-Bean. CH [!, K], CRW $[!, \mathrm{K}, \mathrm{G}]$. Native. OP, CL.

Strophostyles leiosperma (Torr. \& A. Gray) Piper - Slick-Seed FuzzyBean. CH [!, K], CRW [!, K, G]. Native. OP, OC, CL.

Stylosanthes biflora (L.) B.S.P. - Side-Beak Pencil-Flower. CH [!, K], CRW [K, G]. Native. OP.

Tephrosia virginiana (L.) Pers. - Goat's-Rue. CH [!, K], CRW [K, G]. Native. OP.

Trifolium arvense L. - Rabbit-Foot Clover. CH [!]. Non-native. OP. Cherokee County Record (Pryer 5108).

Trifolium campestre Schreb. - Lesser Hop Clover. CH [!, K], CRW [!, K, $\mathrm{G}]$. Non-native. $\mathrm{OP}, \mathrm{OC}, \mathrm{CL}$.

Trifolium carolinianum Michx. - Carolina Clover. CH [!, K]. Native. CL. Was considered extirpated.

Trifolium dubium Sibthorp - Suckling Clover. CH [!, K], CRW [!, K, G]. Non-native. $\mathrm{OP}, \mathrm{CL}$.

Trifolium hybridum L. - Alsike Clover. CH [!]. Non-native. CL. Cherokee County Record (Pryer 155, Pryer 5556).

Trifolium pratense L. - Red Clover. CH [!, K], CRW [!, K, G]. Non-native. $\mathrm{OP}, \mathrm{OC}, \mathrm{CL}$. 
Trifolium reflexum L. - Buffalo Clover. CH [!, K], CRW [!, K, G]. Native. $\mathrm{OP}, \mathrm{OC}, \mathrm{CL}$.

Trifolium repens L. - White Clover. CH [!, K], CRW [!, K, G]. Non-native. $\mathrm{OP}, \mathrm{OC}, \mathrm{CL}$.

Vicia sativa L. subsp. nigra (L.) Ehrh. - Garden Vetch. CH [!]. Nonnative. OP. Cherokee County Record (Pryer 234, Pryer 4186).

Vicia villosa Roth subsp. varia (Host) Corb. - Winter Vetch. CH [K], CRW [!, G]. Non-native. CL.

Vicia villosa Roth subsp. villosa - Winter Vetch. CR [K]. Non-native. Wisteria frutescens (L.) Poir. - American Wisteria. CH [K], CRW [G]. Native. Adventive. One previous collection from 1998 in Crawford County (Timme 15108).

FAGACEAE (Beech Family)

Quercus alba L. - Northern White Oak. CH [!, K], CRW [!]. Native. OP, CL. Crawford County Record (Pryer 5683).

Quercus $\times$ bushii Sarg. - CH [K]. Native.

Quercus $\times$ deamii Trel. - CRW [!]. Native. CL. Crawford County Record (Pryer 3340).

Quercus $\times$ fernowii Trel. $-\mathrm{CH}[\mathrm{K}]$. Native.

Quercus falcata Michx. - Southern Red Oak. CH [!]. Native. OP. State Record. Cherokee County Voucher (Pryer 3054). This taxon is common throughout the southern and eastern states, including the 4-state region. The closest reports are from Ottawa County, Oklahoma and Barry County, Missouri.

Quercus macrocarpa Michx. var. macrocarpa - Burr Oak. CH [!, K], CRW [!, K]. Native. OC, CL. Gibson did not recognize varities of this taxon.

Quercus marilandica (L.) Muenchh. - Blackjack Oak. CH [!, K]. Native. OP.

Quercus michauxii Nutt. - Swamp Chestnut Oak. CH [!]. Native. OP. State Record. Cherokee County Record (Pryer 2951). This taxon is common thoughout the southeastern United States. In Missouri the distribution is restricted to the southeastern portion of the state. It has only been reported in three counties in Oklahoma and throughout Arkanasas. The closest report is from Carroll County, Arkansas. Our one collection is from Schemerhorn Park, part of the Southeast Kansas Nature Center, located on the edge of the Ozark Plateau. The plant occurred in forest between a parking lot along a trail towards an overlook into a cave. We consider it unlikely the tree was planted, given that Schemerhorn is a natural area and tries to manage for native plants. To our knowledge, they have not done any plantings in that area.

Quercus muehlenbergii Engelm. - Chinkapin Oak. CH [!, K], CRW [!, $\mathrm{K}, \mathrm{G}]$. Native. OP, $\mathrm{CL}$.

Quercus palustris Muenchh. - Pin Oak. CH [!, K], CRW [!, K, G]. Native. OC, CL.

Quercus prinoides Willd. - Dwarf Chinkapin Oak. CH [K], CRW [!]. Native. CL. Crawford County Record (Pryer 6181).

Quercus rubra L. - Northern Red Oak. CH [!, K], CRW [!, K, G]. Native. $\mathrm{OP}, \mathrm{CL}$.

Quercus shumardii Buckl. - Shumard's Oak. CH [!, K], CRW [!, K, G]. Native. OC, CL.

Quercus stellata Wangenh. - Post Oak. CH [!, K], CRW [K, G]. Native. OP.

Quercus velutina Lam. - Black Oak. CH [!, K], CRW [K, G]. Native. OP.

\section{GENTIANACEAE (Gentian Family)}

Gentiana alba Muhl. ex Nutt. - Yellow Gentian. CH [K]. Native. Rare. Reported in five counties in Kansas, but common in Missouri; rare in Oklahoma and Arkansas.

Gentiana puberulenta J.S. Pringle - Downy Gentian. CR [!, K]. Native. CL.

Gentianella quinquefolia L. subsp. occidentalis (A. Gray) J. Gillett - SmallAgueweed. CH [K]. Rare. Only reported in Cherokee
County, Kansas, but common in Missouri and Arkansas. This taxon has not been reported in the state of Oklahoma.

Sabatia angularis (L.) Pursh - Rose-Pink. CH [!, K], CRW [!, K, G]. Native.

Rare. OP, CL. Reported in two counties in Kansas, but common in surrounding states.

Sabatia campestris Nutt. - Texas-Star. CH [!, K], CRW [!, K, G]. Native. $\mathrm{OC}, \mathrm{CL}$.

GERANIACEAE (Geranium Family)

Erodium cicutarium (L.) L'Hér. ex Aiton - Red-Stem Stork's-Bill. CRW

[!]. Non-native. OC. Crawford County Record (Pryer 4315).

Geranium carolinianum L. - Carolina Crane's-Bill. CH [!, K], CRW [!, $\mathrm{K}, \mathrm{G}]$. Native. OP, CL.

Geranium maculatum L. - Spotted Crane's-Bill. CH [!, K]. Native. OP. Geranium pusillum L. - Small-Flower Crane's-Bill. CH [K]. Non-native.

GROSSULARIACEAE (Currant Family)

Ribes aureum Pursh var. villosum DC. - Golden Currant. CH [K], CRW [K]. Native.

Ribes missouriense Nutt. - Missouri Gooseberry. CH [!, K], CRW [!, K, G]. Native. OP, OC, CL.

HALORAGACEAE (Water-Milfoil Family)

Myriophyllum aquaticum (Vell.) Verdc. - Parrot's-Feather. CH [!]. Nonnative. CL. Cherokee County Record (Pryer 4938).

Myriophyllum heterophyllum Michx. - Two-Leaf Water-Milfoil. CH [K], CRW [K, G]. Native.

Myriophyllum pinnatum (Walt.) B.S.P. - Cut-Leaf Water-Milfoil. CH [K]. Native.

Myriophyllum sibiricum Komarov - Siberian Water-Milfoil. CH [K], CRW [!, K]. Native. CL.

HEMEROCALLIDACEAE (Day-Lily Family)

Hemerocallis fulva (L.) L. - Orange Day-Lily. CH [!, K], CRW [K]. Non-native. CL.

HYACINTHACEAE (Hyacinth Family)

Muscari botryoides (L.) P. Mill. - Common Grape-Hyacinth. CRW [!, G]. Non-native. $\mathrm{CL}$.

Ornithogalum umbellatum L. - Sleepydick. CH [K], CRW [!, K, G]. Native. OC, CL.

HYDRANGEACEAE (Hydrangea Family)

Hydrangea arborescens L. - Wild Hydrangea. CH [K]. Native. Extirpated.

HYDROCHARITACEAE (Tape-Grass Family)

Najas guadalupensis (Spreng.) Magnus subsp. guadalupensis Guadalupe Waternymph. CH [K], CRW [K]. Native. Gibson did not recognize subspecies of this taxon.

HYPERICACEAE (St. John's-Wort Family)

Hypericum drummondii (Grev. \& Hook.) Torr. \& A. Gray - Nits-andLice. $\mathrm{CH}[\mathrm{K}], \mathrm{CRW}[\mathrm{K}, \mathrm{G}]$. Native.

Hypericum gymnanthum Engelm. \& A. Gray - Clasping-Leaf St. John's-Wort. CRW [!]. Native. CL. State Record. Crawford County Voucher (Pryer 1475, Pryer 5508). This taxon has been reported in most southern states, including Arkansas and Missouri, but is considered rare in Oklahoma. The closest reports are from McDonald and Newton counties, Missouri.

Hypericum hypericoides (L.) Crantz subsp. multicaule (Michx. ex Willd.) Robson - St. Andrew's-Cross. CH [!, K]. Native. Rare. OP. Reported in two counties in Kansas, but common in surrounding states.

Hypericum mutilum L. - Dwarf St. John's-Wort. CH [!, K], CRW [!, K, G]. Native. OP, CL.

Hypericum perforatum L. - Common St. John's-Wort. CH [!, K], CRW $[!, \mathrm{K}, \mathrm{G}]$. Non-native. OC, CL. 
Hypericum punctatum Lam. - Spotted St. John's-Wort. CH [!, K], CRW $[!, \mathrm{K}, \mathrm{G}]$. Native. OP, CL.

Hypericum sphaerocarpum Michx. - Round-Seed St. John's-Wort. $\mathrm{CH}[\mathrm{K}], \mathrm{CRW}[!, \mathrm{K}, \mathrm{G}]$. Native. OC, CL.

HYPOXIDACEAE (Yellow Star-Grass Family)

Hypoxis hirsuta (L.) Coville - Eastern Yellow Star-Grass. CH [!, K], CRW [!, K, G]. Native. OP, OC, CL.

IRIDACEAE (Iris Family)

Belamcanda chinensis (L.) DC. - Blackberry-Lily. CH [!K], CRW [!, K, $\mathrm{G}$. Non-native. $\mathrm{OC}, \mathrm{CL}$.

Crocus vernus (L.) Hill - Dutch Crocus. CRW [!]. Non-native. Found in lawns in Pittsburg, Kansas. Considered a planting and not included in the final tallies.

Iris germanica L. - German Iris. CRW [!, G]. Non-native. CL.

Iris pallida Lam. - Sweet Iris. CRW [!]. Non-native. OC. Crawford County Record (Pryer 4341).

Nemastylis geminiflora Nutt. - Prairie Pleatleaf. CRW [!, K, G]. Native. OC.

Sisyrinchium angustifolium P. Mill. - Narrow-Leaf Blue-Eyed-Grass. $\mathrm{CH}[!, \mathrm{K}], \mathrm{CRW}[!, \mathrm{K}, \mathrm{G}]$. Native. OP, OC, CL.

Sisyrinchium campestre Bickn. - Prairie Blue-Eyed-Grass. CH [!, K], CRW [!, K, G]. Native. OP, OC, CL.

ISOETACEAE (Quillwort Family)

Isoetes butleri Engelm. - Limestone Quillwort. CH [K], CRW [K]. Native.

JUGLANDACEAE (Walnut Family)

Carya $\times$ brownii Sarg. - $\mathrm{CH}[\mathrm{K}]$. Native.

Carya cordiformis (Wangenh.) K. Koch - Bitter-Nut Hickory. CH [!, K], CRW [!, K, G]. Native. OC, CL.

Carya illinoinensis (Wangenh.) K. Koch - Pecan. CH [!, K], CRW [!, K, G]. Native. $\mathrm{OC}, \mathrm{CL}$.

Carya laciniosa (Michx. f.) G. Don - Shell-Bark Hickory. CH [!, K], CRW [K, G]. Native. CL.

Carya ovata (P. Mill.) K. Koch - Shag-Bark Hickory. CH [!, K], CRW [!, $\mathrm{K}, \mathrm{G}$. Native. $\mathrm{OC}, \mathrm{CL}$.

Carya texana Buckl. - Black Hickory. CH [!, K]. Native. Rare. OP. Reported in two counties in Kansas, but common in surrounding states.

Carya tomentosa (Lam. ex Poir.) Nutt. - Mockernut Hickory. CH [!, K], CRW [!]. Native. OP, CL. Crawford County Record (Pryer 2576)

Juglans nigra L. - BlackWalnut. CH [!, K], CRW [!, K, G]. Native. OC, CL.

JUNCACEAE (Rush Family)

Juncus acuminatus Michx. - Knotty-Leaf Rush. CH [!, K], CRW [!, K, G]. Native. OC, CL.

Juncus anthelatus (Wieg.) R.E. Brooks - Kentucky Rush. CH [!, K], CRW [!]. Native. OP, CL. Crawford County Record (Pryer 1451).

Juncus brachycarpus Engelm. - White-Root Rush. CH [!, K], CRW [!,

G]. Native. OC, CL.

Juncus brachyphyllus Wieg. - Short-Leaf Rush. CH [!, K]. CRW [!]. Native. OP, OC, CL. Crawford County Record (Pryer 670, Pryer 672, Pryer 805, Pryer 846, Pryer 1045, and eleven others).

Juncus diffusissimus Buckl. - Slim-Pod Rush. CH [!, K], CRW [!, K, G]. Native. CL.

Juncus dudleyi Wieg. - Dudley's Rush. CH [!, K], CRW [!, G]. Native. $\mathrm{OC}, \mathrm{CL}$.

Juncus effusus L. subsp. solutus (Fernald \& Wieg.) Hämet-Ahti - Lamp Rush. CH [!, K], CRW [!, K, G]. Native. CL.

Juncus interior Wieg. var. interior - Inland Rush. CH [!, K], CRW [!, K] Native. OP, OC, CL.

Juncus marginatus Rostk. - Bog Rush. CH [!, K], CRW [!, K, G]. Native. $\mathrm{CL}$.

Juncus nodatus Coville - Stout Rush. CH [!, K], CRW [!]. Native. Rare.
CL. Crawford County Record (Pryer 1538, Pryer 3662). Reported in ten counties in Kansas, including this report, but common in surrounding states.

Juncus scirpoides Lam. - Needle-Pod Rush. CRW [!]. Native. Rare. CL. Crawford County Record (Pryer 1454). Reported in six counties in Kansas, but common in surrounding states.

Juncus secundus Beauv. ex Poir. - Lopsided Rush. CRW [!]. Native. OC. State Record. Crawford County Record (Pryer 4613). This taxon has been reported throughout the 4-state region. The closest reports are from Barton, Jasper, and McDonald counties Missouri.

Juncus tenuis Willd. - Lesser Poverty Rush. CH [K], CRW [!, K, G]. Native. OC, CL.

Juncus torreyi Coville - Torrey's Rush. CH [!, K], CRW [!, K]. Native. $\mathrm{OC}, \mathrm{CL}$.

Juncus validus Coville - Round-Head Rush. CH [K], CRW [!]. Native. CL. Crawford County Record (Pryer 662). Was considered extirpated.

Luzula bulbosa (Wood) Smyth \& Smyth - Bulbous Wood-Rush. CH [!, K]. Native. OP.

LAMIACEAE (Mint Family)

Agastache nepetoides (L.) Kuntze - Yellow Giant-Hyssop. CH [!, K], CRW [!, K]. Native. OC, CL.

Ajuga reptans L. - Carpet Bugle. CH [K], CRW [K]. Non-native.

Blephilia ciliata (L.) Benth. - Downy Pagoda-Plant. CH [K]. Native.

Callicarpa americana L. - American Beauty-Berry. CRW [!]. Native. CL. State Record. Crawford County Record (Pryer 3656). From two mature bushes located in the middle of a mined land area in Crawford County. The area was remote and the plants did not appear to have been introduced. This taxon is common throughout the southeastern United States, including Arkansas and southern Oklahoma. It is considered rare in Missouri. The closest reports are from Pawnee County, Oklahoma and Taney County, Missouri.

Cunila origanoides (L.) Britton - Common Dittany. CH [!, K]. Native. Rare. OP. Only reported in Cherokee County, Kansas, but common in surrounding states.

Glechoma hederacea L. - Groundivy. CH [!], CRW [!]. Non-native. OP, CL. Cherokee County Record (Pryer 4195). Crawford County Record (Pryer 512, Pryer 4250, Snow 10,772, Snow 11098).

Hedeoma hispida Pursh - Rough False Pennyroyal. CH [!, K], CRW [!, K, G]. Native. OC, CL.

Hedeoma pulegioides (L.) Pers. - American False Pennyroyal. CH [K]. Native.

Lamium amplexicaule L. - Giraffehead. CH [K], CRW [!, K, G]. Nonnative. $\mathrm{OC}, \mathrm{CL}$.

Lamium purpureum L. var. purpureum - Red Henbit. CH [!, K], CRW $[!, K, G]$. Non-native. OC, CL. Gibson did not recognize varieties for this taxon.

Leonurus cardiaca L. subsp. cardiaca - Motherwort. CH [K]. Non-Naitve.

Lycopus americanus Muhl. ex W.P.C. Barton - Cut-Leaf WaterHorehound. CH [!, K], CRW [!, K, G]. Native. OC, CL.

Lycopus rubellus Moench - Taper-Leaf Water-Horehound. CH [K]. Native. Rare. Reported in three counties in Kansas, but common in surrounding states.

Lycopus virginicus L. - Virginia Water-Horehound. CH [K], CRW [G]. Native.

Marrubium vulgare L. - White Horehound. CH [K]. Non-native.

Mentha arvensis L. - American Wild Mint. CH [K], CRW [G]. Native.

Monarda bradburiana Beck - Eastern Beebalm. CH [!, K]. Native.

Rare. OP. Reported in two counties in Kansas, but common in surrounding states.

Monarda citriodora Cerv. ex Lag. subsp. citriodora-Lemon Beebalm. 
CRW [!, G]. Native. OC, CL. Gibson did not recognize varieties for this taxon.

Monarda fistulosa L. subsp. fistulosa - Oswego-Tea. CH [!, K], CRW $[!, K, G]$. Native. Native. CL. Gibson did not recognize subspecies for this taxon.

Nepeta cataria L. - Catnip. CH [K], CRW [!, G]. Non-native. OC.

Perilla frutescens (L.) Britton var. frutescens - Beefsteakplant. CH [!, $\mathrm{K}]$, CRW [!, K]. Non-native. OP, OC, CL.

Physostegia angustifolia Fernald - Narrow-Leaf False Dragonhead. CH [!, K], CRW [!, K, G]. Native. Rare. OC, CL. Reported in seventeen counties in Kansas, but common in surrounding states.

Physostegia virginiana (L.) Benth. subsp. praemorsa (Shinners) Cantino - Obedient-Plant. CH [K], CRW [K]. Native.

Physostegia virginiana (L.) Benth. subsp. virginiana - Obedient-Plant. CH [!], CRW [!]. Native. OP. Cherokee County Record (Pryer 1075, Pryer 4973, Pryer 5343b). Crawford County Record (Pryer 5305).

Prunella vulgaris L. subsp. lanceolata (W.P.C. Barton) Hultén Common Selfheal. CH [!, K], CRW [!, K, G]. Native. OC, CL.

Pycnanthemum albescens Torr. \& A. Gray - White-Leaf MountainMint. CH [K]. Native. Extirpated. The most recent specimen located was collected on August 21, 1949 (R.L. McGregor 3850) for Cherokee County, Kansas. This specimen resides at McGregor Herbarium, University of Kansas.

Pycnanthemum tenuifolium Schrad. - Narrow-Leaf Mountain-Mint. $\mathrm{CH}[!, \mathrm{K}], \mathrm{CRW}[!, \mathrm{K}, \mathrm{G}]$. Native. OP, OC, CL.

Pycnanthemum verticillatum (Michx.) Pers. var. pilosum (Nutt.) Cooperrider - Whorled Mountain-Mint. CH [!, K]. Native. OP.

Pycnanthemum virginianum (L.) T. Dur. \& B.D. Jackson ex B.L. Rob. \& Fernald - Virginia Mountain-Mint. CH [K]. Native.

Salvia azurea Michx. ex Lam. var. grandiflora Benth. - Azure-Blue Sage. CH [!, K], CRW [!, K, G]. Native. OC, CL.

Salvia farinacea Benth. - Mealy-Cup Sage. CRW [!]. Native. CL. State Record. Crawford County Voucher (Pryer 3901). Several (10+) plants located on a mined land area next to a strip pit. One specimen was collected in Neosho County, Kansas, but was noted as "under cultivation" (W.W. Holland 5857; September 4, 1987). The specimen is held in the McGregor Herbarium, University of Kansas. This taxon has only been reported in New Mexico, Texas, Louisiana, Oklahoma, Florida, Ohio, and Connecticut. The closest report is from Delaware County, Oklahoma.

Scutellaria incana Biehler - Hoary Skullcap. CH [!, K]. Native. Rare. OP. Only reported in Cherokee county; common in Missouri and Arkansas but rare in Oklahoma.

Scutellaria lateriflora L. var. lateriflora - Mad Dog Skullcap. CH [!, K], CRW [K]. Native. OP.

Scutellaria ovata Hill subsp. ovata - Heart-Leaf Skullcap.

Scutellaria parvula Michx. - Small Skullcap. CH [!, K], CRW [!, K, G]. Native. OP, OC, CL.

Stachys pilosa Nutt. var. pilosa-Hairy Hedge-Nettle. CRW [!]. Native. Rare. OP. Crawford County Record (Pryer 1334, Pryer 3636). Reported in six counties in Kansas, but rare in surrounding states and not yet reported for Arkansas.

Stachys tenuifolia Willd. - Smooth Hedge-Nettle. CH [!, K], CRW [!, $\mathrm{K}, \mathrm{G}$. Native. $\mathrm{OC}, \mathrm{CL}$.

Teucrium canadense $\mathrm{L}$. var. canadense - American Germander. $\mathrm{CH}$ $[!, \mathrm{K}], \mathrm{CRW}[!, \mathrm{K}, \mathrm{G}]$. Native. OC, CL.

Teucrium canadense L. var. occidentale (A. Gray) McClintock \& Epling - American Germander. CRW [!]. Native. CL. Crawford County Record (Pryer 2152).

Trichostema brachiatum L. - False Pennyroyal. CRW [K, G]. Native.

LAURACEAE (Laurel Family)

Lindera benzoin (L.) Blume - Northern Spicebush. CH [!, K], CRW [!, $\mathrm{K}, \mathrm{G}$. Native. OP, CL.

Sassafras albidum (Nutt.) Nees - Sassafras. CH [!, K], CRW [K]. Native. OP.
LENTIBULARIACEAE (Bladderwort Family)

Utricularia gibba L. - Humped Bladderwort. CH [K], CRW [!, K, G]. Native. CL.

Utricularia macrorhiza Le Conte - Greater Bladderwort. CRW [!]. Native. CL. Crawford County Record (Pryer 2582).

LILIACEAE (Lily Family)

Erythronium albidum Nutt. - Small White Fawn-Lily. CH [K], CRW [!, K]. Native. CL.

Erythronium mesochoreum Knerr - Midland Fawn-Lily. CH [!, K], CRW [K, G]. Native. CL.

Erythronium rostratum W. Wolf - Yellow Trout-Lily. CH [K]. Native. Rare. Only reported in Cherokee County, Kansas, but common in surrounding states.

Lilium michiganense Farw. - Michigan Lily. CH [K], CRW [!, K, G]. Native. Rare. Reported in eleven counties in Kansas; common in Missouri and Arkansas but with only one county report in Oklahoma.

\section{LINACEAE (Flax Family)}

Linum medium (Planch.) Britton var. texanum (Planch.) Fernald - Stiff Yellow Flax. CH [K], CRW [!, K]. Native. Rare. CL. Reported in three counties in Kansas but common in surrounding states.

Linum sulcatum Riddell var. sulcatum - Grooved Yellow Flax. $\mathrm{CH}$ [K], CRW [!, K, G]. Native. OC, CL. Gibson did not recognize varieties of this taxon.

Linum usitatissimum L. - Cultivated Flax. CR [K, G]. Non-native.

LINDERNIACEAE (False Pimpernel Family)

Lindernia dubia (L.) Pennell - Yellow-Seed False Pimpernel. CH [!, K], CRW [!, K, G]. Native. OP, CL. Variety was not able to be determined. Kartesz (2017) recognizes Lindernia dubia (L.) var. anagallidea (Michx.) $\mathrm{CH}[\mathrm{K}]$.

LOASACEAE (Blazingstar Family)

Mentzelia albescens (Gillies \& Arn.) Griseb. - Wavy-Leaf Blazingstar. $\mathrm{CH}[\mathrm{K}]$. Native. Adventive.

Mentzelia oligosperma Nutt. ex Sims - Chickenthief. CH [K]. Native.

LYTHRACEAE (Loosestrife Family)

Ammannia coccinea Rottb. - Valley Redstem. CH [!, K], CRW [!, K, G]. Native. OC, CL.

Ammannia robusta Heer \& Regel - Grand Redstem. CH [!, K], CRW [!]. Native. OC, CL. Crawford County Record (Pryer 5613).

Cuphea viscosissima Jacq. - Blue Waxweed. CH [!, K], CRW [!, K, G]. Native. OP, OC, $\mathrm{CL}$.

Didiplis diandra (Nutt. ex DC.) Wood - Water-Purslane. CH [K], CRW $[K, G]$. Naitve. Rare. Reported in nine counties in Kansas; common in Missouri but rare in Oklahoma and Arkansas.

Lythrum alatum PurshWing-Angle var. alatum - Loosestrife. CH [K], CRW [!, K, G]. Native. CL. Gibson did not recognize varieties of this taxon.

Lythrum californicum Torr. \& A. Gray - California Loosestrife. CRW [K]. Native.

Rotala ramosior (L.) Koehne - Lowland Toothcup. CH [!, K], CRW [!, K]. Native. OP, CL.

MALVACEAE (Mallow Family)

Abutilon theophrasti Medik. - Velvetleaf. CH [K], CRW [!, K, G]. Nonnative. Noxious. $\mathrm{OC}, \mathrm{CL}$.

Callirhoe alcaeoides (Michx.) A. Gray - Light Poppy-Mallow. CH [!, $\mathrm{K}], \mathrm{CRW}[!, \mathrm{K}, \mathrm{G}]$. Native. OP, OC, CL.

Callirhoe digitata Nutt. - Winecup. CH [K]. Native. Rare. Reported in two counties in Kansas but common in surrounding states.

Hibiscus laevis All. - Halberd-Leaf Rose-Mallow. CH [!, K], CRW [K, G]. Native. CL.

Hibiscus moscheutos L. subsp. lasiocarpos (Cav.) O.J. Blanchard Crimson-Eye Rose-Mallow. CH [!, K], CRW [!, K]. Native. OP, CL. 
Hibiscus moscheutos L. subsp. moscheutos - Crimson-Eye RoseMallow. $\mathrm{CH}[\mathrm{K}], \mathrm{CRW}[\mathrm{K}, \mathrm{G}]$. Native.

Hibiscus trionum L. - Flower-of-An-Hour. CH [K], CRW [!, K, G]. Native. Non-native. CL.

Malva neglecta Wallr. - Dwarf Mallow. CH [K], CRW [K, G]. Non-native.

Malva pusilla Sm. - Low Mallow CH [K]. Non-native.

Malvastrum angustum A. Gray - Hispid False Mallow. CRW [K]. Native.

Sida spinosa L. - Prickly Fanpetals. CH [!, K], CRW [!, K, G]. Native. Adventive. OC, CL.

Tilia americana L. var. americana - American Basswood. CRW [K]. Gibson. Native.

MARSILEACEAE (Water-Clover Family)

Marsilea quadrifolia L. - European Water-Clover. CH [K]. Non-native. Marsilea vestita Hook. \& Grev. - Hairy Water-Clover. CH [K]. Native.

MELANTHIACEAE (False Hellebore Family)

Veratrum virginicum (L.) W.T. Aiton - Virginia Bunchflower. CRW [K,

G]. Native. Rare. Reported in seven counties in Kansas; common in Missouri and Arkansas, but rare in Oklahoma.

MELASTOMATACEAE (Melastome Family)

Rhexia mariana L. var. interior (Pennell) Kral \& Bostick - Maryland Meadow-Beauty. CH [!, K], CRW [!, K, G]. Native. Rare. Reported in two counties in Kansas but common in surrounding states.

MENISPERMACEAE (Moonseed Family)

Calycocarpum lyonii (Pursh) A. Gray-Cupseed. CH [K]. Native. Rare. Reported in three counties in Kansas; common in Missouri and Arkansas but rare in Oklahoma.

Cocculus carolinus (L.) DC. - Carolina Coralbead. CH [!, K], CRW [!, K, G]. Native. OP, OC, CL.

Menispermum canadense L. - Canadian Moonseed. CH [!, K], CRW $[!, \mathrm{K}, \mathrm{G}]$. Native. OP, OC, CL.

MOLLUGINACEAE (Carpetweed Family)

Glinus lotoides L. - Lotus Sweetjuice. CRW [K]. Non-native.

Mollugo verticillata L. - Green Carpetweed. CH [!, K], CRW [!, K, G]. Native. Adventive. OC, CL.

MONTIACEAE (Candy-Flower Family)

Claytonia virginica L. var. virginica - Virginia Springbeauty. CH [!, K], CRW [!, K, G]. Native. OP, OC, CL.

Phemeranthus parviflorus (Nutt.) Kiger - Prairie False Fameflower. $\mathrm{CH}[\mathrm{K}], \mathrm{CRW}[!, \mathrm{K}, \mathrm{G}]$. Native.

MORACEAE (Mulberry Family)

Fatoua villosa (Thunb.) Nakai - Hairy Crabweed. CRW [!]. Non-native. CL. Crawford County Record (Pryer 2141, Pryer 3246).

Maclura pomifera (Raf.) Schneid. - Osage-Orange. CH [!, K], CRW [!, $\mathrm{K}, \mathrm{G}]$. Native. Adventive. OC, CL.

Morus alba L. - White Mulberry. CH [!, K], CRW [!, K, G]. Non-native. $\mathrm{OP}, \mathrm{OC}, \mathrm{CL}$.

Morus rubra L. - Red Mulberry. CH [!, K], CRW [!, K, G]. Native. OP, $\mathrm{OC}, \mathrm{CL}$.

NELUMBONACEAE (Lotus-Lily Family)

Nelumbo lutea Willd. - American Lotus. CH [!, K], CRW [!, K]. Native. $\mathrm{OP}, \mathrm{CL}$.

NYCTAGINACEAE (Four-O'clock Family)

Mirabilis albida (Walt.) Heimerl - White Four-O'clock. CH [K], CRW $[\mathrm{K}, \mathrm{G}]$. Native.

Mirabilis linearis (Pursh) Heimerl var. linearis - Narrow-Leaf FourO'clock. CRW [!, K]. Native. OC.

Mirabilis nyctaginea (Michx.) MacMill. - Heart-Leaf Four-O'clock. CH [!, K], CRW [!, K, G]. Native. OP, OC, CL.
NYMPHAEACEAE (Water-Lily Family)

Nuphar advena (Aiton) W.T. Aiton subsp. advena - Yellow Pond-Lily. $\mathrm{CH}[\mathrm{K}], \mathrm{CRW}$ [!, K]. Native. Rare. CL. Reported in six counties in Kansas but common in surrounding states.

Nymphaea odorata Aiton subsp. odorata - American White WaterLily. $\mathrm{CH}[\mathrm{K}], \mathrm{CRW}[\mathrm{K}, \mathrm{G}]$. Native. Gibson did not recognize subspecies of this taxon.

OLEACEAE (Olive Family)

Forestiera acuminata (Michx.) Poir. - Eastern Swamp-Privet. CH [!, K]. Native. Rare. OP. Reported in two counties in Kansas; common in Arkansas and parts of Missouri but rare in Oklahoma.

Forsythia suspensa (Thunb.) Vahl - Weeping Forsythia. CH [!]. Nonnative. CL. Probable planting at an old homestead. Included here for reference, but not included in any tallies.

Fraxinus americana L. - White Ash. CH [K], CRW [K, G]. Native.

Fraxinus pennsylvanica Marshall - Green Ash. CH [!, K], CRW [!, K, G]. Native. OC, $\mathrm{CL}$.

Fraxinus quadrangulata Michx. - Blue Ash. CH [K], CRW [K]. Native. Ligustrum obtusifolium Siebold \& Zucc. subsp. obtusifolium - Border Privet. CH [K], CRW [!]. Non-native. CL. Crawford County Record (Pryer 734, Pryer 746, Pryer 783, Pryer 2229).

Ligustrum sinense Lour. - Chinese Privet. CH [!], CRW [!]. Non-native. OP, OC, CL. Cherokee County Record (Pryer 3169), Crawford County Record (Pryer 2744, Pryer 3285, Pryer 3355).

Ligustrum vulgare L. - European Privet. CRW [!, G]. Non-native. OC. Syringa vulgaris L. - Common Lilac. CH [!]. Non-native. CL. Probably originally planted near an old homestead, though obviously naturalizing at this point. State Record (Pryer 4023).

ONAGRACEAE (Evening-Primrose Family)

Circaea canadensis (L.) Hill - Broad-Leaf Enchanter's-Nightshade. CH [K], CRW [!]. Native. OC. Crawford County Record (Pryer 1311, Pryer 1322, Pryer 2005, Pryer 5427).

Ludwigia alternifolia L. - Seedbox. CH [!, K], CRW [!, K, G]. Native. $\mathrm{OP}, \mathrm{CL}$.

Ludwigia glandulosa Walt. subsp. glandulosa - Cylindric-Fruit Primrose-Willow. $\mathrm{CH}[\mathrm{K}]$. Native.

Ludwigia palustris (L.) Elliott - Marsh Primrose-Willow. CH [!, K], CRW $[!, \mathrm{K}, \mathrm{G}]$. Native. OP, CL.

Ludwigia peploides (Kunth) subsp. glabrescens (Kuntze) - Raven RavenFloating Primrose-Willow. CH [!, K], CRW [!, K, G]. Native. CL.

Oenothera biennis L. - King's-Cureall. CRW [K]. Native.

Oenothera curtiflora W.L. Wagner \& Hoch - Velvetweed. CRW [G]. Native.

Oenothera filiformis (Small) W.L. Wagner \& Hoch - Long-Flower Evening-Primrose. CH [!, K], CRW [!, K, G]. Native. OC, CL.

Oenothera laciniata Hill - Cut-Leaf Evening-Primrose. CH [!, K], CRW $[!, \mathrm{K}, \mathrm{G}]$. Native. OC, CL.

Oenothera linifolia Nutt. - Thread-Leaf Evening-Primrose. CH [K], CRW [!, G]. Native. OC.

Oenothera macrocarpa Nutt. subsp. macrocarpa-Big-Fruit EveningPrimrose. CH [K], CRW [!, K, G]. Native. OC.

Oenothera speciosa Nutt. - Pinkladies. CH [!, K], CRW [!, K, G]. Native. OC, CL.

Oenothera triloba Nutt. - Stemless Evening-Primrose. CH [!, K], CRW [!, K, G]. Native. CL.

Oenothera villosa Thunb. subsp. villosa - Hairy Evening-Primrose. $\mathrm{CH}[!, \mathrm{K}], \mathrm{CRW}[$ [!, K]. Native. OP, OC, CL.

ORCHIDACEAE (Orchid Family)

Aplectrum hyemale (Muhl. ex Willd.) Torr. - Adam-and-Eve. CRW [!]. Native.

Calopogon oklahomensis D.H. Goldman - Oklahoma Grass-Pink. CH [K]. Native. Rare. Only reported in Cherokee County, Kansas; 
in the Midwest south to the Gulf Coast, but considered rare wherever it occurs.

Corallorhiza odontorhiza (Willd.) Poir. - Autumn Coralroot. CH [K], CRW [K]. Native.

Corallorhiza wisteriana Conrad - Spring Coralroot. CH [K]. Native.

Cypripedium parviflorum Salisb. var. parviflorum - Yellow Lady'sSlipper. $\mathrm{CH}$ [K]. Native.

Malaxis unifolia Michx. - Green Adder's-Mouth Orchid. CH [K]. Native. Rare. Reported in two counties in Kansas; common in Missouri and Arkansas but rare in Oklahoma.

Platanthera lacera (Michx.) G. Don - Green Fringed Orchid. CH [!, $\mathrm{K}]$, CRW [!, K]. Native. Rare. CL. Reported in eight counties in Kansas; common in Missouri and Arkansas but rare in Oklahoma.

Platanthera praeclara Sheviak \& Bowles - Great Plains White Fringed Orchid. CRW [K, G]. Native. Rare. Federally listed as threatened. Reported in fourteen counties in Kansas. This taxon in considered rare wherever it occurs. It has been reported throughout eastern Missouri and two counties in Oklahoma, but is considered extirpated from the state of Oklahoma.

Spiranthes cernua (L.) L.C. Rich. - White Nodding Ladies'-Tresses. CRW [!, G]. Native. CL. Reported for Kansas first by Gibson (1963) for Crawford County, and here confirmed also for Allen, Bourbon and Neosho counties: Allen Co. (W.H. Horr E429 \& R. L. McGregor [KSP024418]); Bourbon Co. (Stoughton s.n. [KSP001306]); Crawford Co. (E. B. Holmes s.n. [KSP024421]; E.S. Gibson s.n. [KSP024420]; Pryer 6409, Pryer 6410); a collection from Sperry's residence in Pittsburg (Sperry P0224 [KSP024419]) cannot be confirmed with confidence, as it is uncertain which of the dozens of species collected from that property were transplanted); Neosho Co. (Holland 3209 [KSP024416]). All identifications except that for Bourbon County were made in December 1987 by L.K. Magrath, a specialist of Great Plains orchids for many years.

Spiranthes lacera (Raf.) Raf. - Northern Slender Ladies'-Tresses. CH [!, K], CRW [!, K]. Native. CL. Variety could not be determined in Kansas specimens since none of the identification texts used recognized varities.

Spiranthes lacera (Raf.) Raf. var. gracilis (Bigelow) Luer - Northern Slender Ladies'-Tresses. CH [K], CRW [K]. Native.

Spiranthes lucida (H.H. Eaton) Ames - Shining Ladies'-Tresses. $\mathrm{CH}$ [!]. Native. CL. Was considered extirpated. Cherokee County Record (Pryer 5419).

Spiranthes magnicamporum Sheviak - Great Plains Ladies'-Tresses. CH [!, K], CRW [!]. Native. CL. Crawford County Record (Pryer 1926, Pryer 3690).

Spiranthes tuberosa Raf. - Little Ladies'-Tresses. CH [K]. Native.

Spiranthes vernalis Engelm. \& A. Gray - Spring Ladies'-Tresses. $\mathrm{CH}$ [!, K], CRW [!, K, G]. Native. OC, CL.

OROBANCHACEAE (Broom-Rape Family)

Agalinis aspera (Dougl. ex Benth.) Britton - Tall False Foxglove. $\mathrm{CH}$ [K], CRW [!, K]. Native. CL.

Agalinis auriculata (Michx.) Blake - Ear-Leaf False Foxglove. CRW [K]. Native. Rare. Reported in eighteen counties in Kansas, but considered rare wherever it is reported. The most concentrated distribution is in Kansas, Missouri, lowa, and Illinois.

Agalinis fasciculata (Elliott) Raf. - Beach False Foxglove. CH [!, K], CRW [!, K, G]. Native. Rare. OP, CL. Reported in five counties in Kansas but common in surrounding states.

Agalinis gattingeri (Small) Small - Round-Stem False Foxglove. $\mathrm{CH}$ [!, K]. Native. OP.

Agalinis heterophylla (Nutt.) Small ex Britton - Prairie False Foxglove. $\mathrm{CH}[!, \mathrm{K}]$. Native. Rare. OP. Reported in six counties in Kansas; common in Oklahoma and Arkansas but rare in Missouri.
Agalinis purpurea (L.) Pennell - Purple False Foxglove. CH [K], CRW [!, K, G]. Native. Rare. CL.

Agalinis skinneriana (Wood) Britton - Skinner's False Foxglove. $\mathrm{CH}[\mathrm{K}]$. Native.

Agalinis tenuifolia (Vahl) Raf. - Slender-Leaf False Foxglove. CH [!, K], CRW [!, K, G]. Native. CL.

Aureolaria grandiflora (Benth.) Pennell var. serrata (Torr. ex Benth.) Pennell - Large-Flower Yellow False Foxglove. CH [!, K], CRW [!]. Native. Rare. OP, OC. Crawford County Record (Pryer 2770). Reported in three counties in Kansas, including this report but common in surrounding states.

Buchnera americana L. - American Bluehearts. CH [!, K], CRW [!, K, G]. Native. OC, $\mathrm{CL}$.

Castilleja coccinea (L.) Spreng. - Scarlet Indian-Paintbrush. CH [!, K], CRW [!, K, G]. Native. OC, CL.

Dasistoma macrophylla (Nutt.) Raf. - Mullein-Foxglove. CH [K], CRW [!, K, G]. Native. OC, CL.

Orobanche uniflora L. - Naked Broomrape. CH [K]. Native.

Pedicularis canadensis L. - Canadian Lousewort. CH [!, K], CRW [!, $\mathrm{K}, \mathrm{G}]$. Native. OP, OC.

OXALIDACEAE (Wood-Sorrel Family)

Oxalis corniculata L. - Creeping Yellow Wood-Sorrel. CH [!, K], CRW $[!, \mathrm{G}]$. Native. Adventive. OP, CL.

Oxalis dillenii Jacq. - Slender Yellow Wood-Sorrel. CH [!, K], CRW [!, K]. Native. OP, OC, CL.

Oxalis stricta L. - Upright Yellow Wood-Sorrel. CH [!, K], CRW [!, K, G]. Native. OP, OC, CL.

Oxalis violacea L. - Violet Wood-Sorrel. CH [!, K], CRW [!, K, G]. Native. $\mathrm{OP}, \mathrm{OC}, \mathrm{CL}$.

PAPAVERACEAE (Poppy Family)

Corydalis crystallina Engelm. - Mealy Fumewort. CH [K], CRW [K]. Native.

Corydalis flavula (Raf.) DC. - Yellow Fumewort. CH [!, K], CRW [!, K]. Native. OP, CL.

Corydalis micrantha (Engelm. ex A. Gray) A. Gray subsp. australis (Chapman) G.B. Ownbey - Small-Flower Fumewort. CH [K], CRW [K, G]. Native.

Corydalis micrantha (Engelm. ex A. Gray) A. Gray subsp. micranthaSmall-Flower Fumewort. CRW [!]. Native. CL. Crawford County Record (Pryer 92, Pryer 4351, Pryer 4516).

Dicentra cucullaria (L.) Bernh. - Dutchman's-Breeches. CH [!, K], CRW $[!, \mathrm{K}, \mathrm{G}]$. Native. OP, OC, CL.

Papaver rhoeas L. - Corn Poppy. CH [K]. Non-native.

Sanguinaria canadensis L. - Bloodroot. CH [!, K]. Native. OP.

PASSIFLORACEAE (Passion-Flower Family)

Passiflora incarnata L. - Purple Passion-Flower. CH [!, K], CRW [!, K, G]. Native. OP, CL.

Passiflora lutea L. - Yellow Passion-Flower. CH [!, K], CRW [K, G]. Native. Rare. OP. Reported in four counties in Kansas, but common in surrounding states.

PENTHORACEAE (Ditch-Stonecrop Family)

Penthorum sedoides L. - Ditch-Stonecrop. CH [!, K], CRW [!, K, G]. Native. OC, CL.

PHRYMACEAE (Lopseed Family)

Mimulus alatus Aiton - Sharp-Wing Monkey-Flower. CH [K], CRW $[!, \mathrm{K}, \mathrm{G}]$. Native. OC, CL.

Phryma leptostachya L. - American Lopseed. CH [!, K], CRW [!, K, G]. Native. $\mathrm{OP}, \mathrm{OC}, \mathrm{CL}$.

PHYLLANTHACEAE (Leaf-Flower Family)

Phyllanthus caroliniensis Walt. subsp. caroliniensis - Carolina LeafFlower. CH [K], CRW [K]. Native. Rare. Gibson did not recognize 
subspecies of this taxon. Reported in five counties in Kansas but common in surrounding states.

PHYTOLACCACEAE (Pokeweed Family)

Phytolacca americana L. var. americana - American Pokeweed. $\mathrm{CH}[!, \mathrm{K}], \mathrm{CRW}[!, \mathrm{K}, \mathrm{G}]$. Native. CL. Gibson did not recognize varieties of this taxon.

PLANTAGINACEAE (Plantain Family)

Callitriche heterophylla Pursh subsp. heterophylla - Greater WaterStarwort. CH [!, K], CRW [!, K, G]. Native. CL.

Callitriche terrestris Raf. - Terrestrial Water-Starwort. CH [K]. Native. Rare. Reported in seven counties in Kansas; common in Missouri and Arkansas but rare in Oklahoma.

Chaenorhinum minus (L.) - LangeDwarf-Snapdragon. CH [!, K]. Non-native. OP.

Collinsia violacea Nutt. - Violet Blue-Eyed Mary. CH [!, K], CRW [!, K, G]. Native. $\mathrm{OC}, \mathrm{CL}$.

Gratiola neglecta Torr. - Clammy Hedge-Hyssop. CH [!, K], CRW [K, G]. Native. CL.

Gratiola virginiana L. var. virginiana - Round-Fruit Hedge-Hyssop. CRW [G]. Native.

Leucospora multifida (Michx.) Nutt. - Narrow-Leaf Paleseed. CH [K], CRW [!, K, G]. Native. OC, CL.

Linaria vulgaris P. Mill. - Greater Butter-and-Eggs. CH [K], CRW [!, K, G]. Native. Non-native. CL.

Mecardonia acuminata (Walt.) Small var. acuminata - Axil-Flower. $\mathrm{CH}[\mathrm{K}]$. Native. Rare. Only reported in Cherokee County, Kansas; common in Oklahoma and Arkansas but rare in Missouri.

Nuttallanthus canadensis (L.) D.A. Sutton - Oldfield-Toadflax. CH [!], CRW [K]. Native. OP. Cherokee County Record (Pryer 5105).

Nuttallanthus texanus (Scheele) D.A. Sutton - Texas-Toadflax. CH [!, $\mathrm{K}], \mathrm{CRW}[!, \mathrm{K}, \mathrm{G}]$. Native. OP, CL.

Penstemon digitalis Nutt. ex Sims - Foxglove Beardtongue. CH [!, K], CRW [!, K, G]. Native. OC, CL.

Penstemon tubiflorus Nutt. var. tubiflorus - White Wand Beardtongue. $\mathrm{CH}[!, \mathrm{K}], \mathrm{CRW}[!, \mathrm{K}, \mathrm{G}]$. Native. OC, CL. Gibson did not recognize varieties for this taxon.

Plantago aristata Michx. - Large-Bract Plantain. CH [!, K], CRW [!, K, G]. Native. OP, OC, CL.

Plantago elongata Pursh - Prairie Plantain. CH [K]. Native.

Plantago lanceolata L. - English Plantain. CH [!, K], CRW [!, K, G]. Non-native. Noxious. OC, CL.

Plantago major L. - Great Plantain. CH [!, K], CRW [K, G]. Nonnative. OP.

Plantago patagonica Jacq. - Woolly Plantain. CH [K], CRW [K]. Native.

Plantago rhodosperma Decne. - Red-Seed Plantain. CH [!], CRW [!]. Native. OP, OC. Cherokee County Record (Pryer 426). Crawford County Record (Pryer 4841, Pryer 4918).

Plantago rugelii Decne. - Black-Seed Plantain. CH [!, K], CRW [!, K, G]. Native. OP, OC, CL.

Plantago virginica L. - Pale-Seed Plantain. CH [!, K], CRW [!, K, G]. Native. OP, OC, CL.

Plantago wrightiana Decne. - Wright's Plantain. CRW [K]. Native.

Veronica agrestis L. - Green Field Speedwell. CH [K], CRW [K]. Non-native.

Veronica arvensis L. - Corn Speedwell. CH [!, K], CRW [!, K, G]. Nonnative. $\mathrm{OP}, \mathrm{OC}, \mathrm{CL}$.

Veronica peregrina L. subsp. peregrine - Neckweed. CH [!, K], CRW $[!, \mathrm{K}]$. Native. OP, OC, CL.

Veronica peregrina L. subsp.xalapensis (Kunth) Pennell - Neckweed. $\mathrm{CH}[!, \mathrm{K}], \mathrm{CRW}[\mathrm{K}, \mathrm{G}]$. Native. CL.

Veronica polita Fr. - Field Speedwell. CH [!, K], CRW [!, K, G]. Nonnative. $\mathrm{OP}, \mathrm{OC}, \mathrm{CL}$.

Veronicastrum virginicum (L.) Farw. - Culver's-Root. CH [K]. Native.
PLATANACEAE (Planetree Family)

Platanus occidentalis L. - American Sycamore. CH [!, K], CRW [!, K, G]. Native. OP, OC, CL.

POACEAE (Grass Family)

Aegilops cylindrica Host - Jointed Goat Grass. CH [K], CRW [K, G]. Non-native. Noxious.

Agrostis elliottiana J.A. Schultes - Elliott's Bent. CH [K], CRW [K, G]. Native. Rare. Reported in eleven counties in Kansas but common in surrounding states.

Agrostis gigantea Roth - Black Bent. CH [K], CRW [!, K, G]. Nonnative. OC.

Agrostis hyemalis (Walt.) B.S.P. - Winter Bent. CH [!, K], CRW [!, K, G]. Native. OP, OC, $\mathrm{CL}$.

Agrostis perennans (Walt.) Tuckerman - Upland Bent. CH [!, K], CRW $[!, \mathrm{K}, \mathrm{G}]$. Native. OP, OC, CL.

Agrostis scabra Willd. - Rough Bent. CH [K]. Native. Rare. Only reported in Cherokee County, Kansas, and rare in all surrounding states.

Agrostis stolonifera L. - Spreading Bent. CH [K], CRW [!, K]. Nonnative. $\mathrm{CL}$

Alopecurus carolinianus Walt. - Tufted Meadow-Foxtail. CH [!, K], CRW [K, G]. Native.

Andropogon gerardi Vitman - Big Bluestem. CH [!, K], CRW [!, K, G]. Native. OC, CL.

Andropogon ternarius Michx. - Split-Beard Bluestem. CH [!, K], CRW $[!, \mathrm{K}]$. Native. OP, CL.

Andropogon virginicus L. var. virginicus - Broom-Sedge $\mathrm{CH}[!, \mathrm{K}]$, CRW [!, K, G]. Native. OC, CL. Gibson did not recognize varieties of this taxon.

Aristida adscensionis L. - Six-Weeks Three-Awn. CH [!]. Native. CL. Cherokee County Record (Pryer 5054).

Aristida basiramea Engelm. ex Vasey - Forked Three-Awn. CH [K], CRW [!]. Native. CL. Crawford County Record (Pryer 6081).

Aristida dichotoma Michx. var. curtissii A. Gray ex S. Wats. \& Coult. Church-Mouse Three-Awn. CH [K], CRW [K]. Native.

Aristida dichotoma Michx. var. dichotoma - Church-Mouse ThreeAwn. $\mathrm{CH}[\mathrm{K}], \mathrm{CRW}[$ [!, K]. Native. CL.

Aristida longespica Poir. var. geniculata (Raf.) Fernald - Red ThreeAwn. CRW [!]. Native. CL. Crawford County Record (Pryer 6089).

Aristida longespica Poir. var. longespica - Red Three-Awn. CH [K], CRW [K]. Native.

Aristida oligantha Michx. - Prairie Three-Awn. CH [!, K], CRW [!, K, G]. Native. OP, OC, CL.

Aristida purpurascens Poir. var. purpurascens - Arrow-FeatherThreeAwn. CH [!, K]. Native. OP, CL.

Aristida ramosissima Engelm. ex A. Gray - S-Curve Three-Awn. CH [!, K]. Native. Rare. CL. Only reported in Cherokee County, Kansas but rare in the immediately surrounding area and considered extirpated from Missouri.

Arthraxon hispidus (Thunb.) Makino - Small Carp Grass. CH [K], CRW [!]. Non-native. CL. Crawford County Record (Pryer 3666).

Bothriochloa bladhii (Retz.) S.T. Blake - Australian Beard Grass. CH [!]. Non-native. OP. Cherokee County Record (Pryer 5784).

Bothriochloa ischaemum (L.) Keng - Turkestan Beard Grass. CH [!], CRW [!]. Non-Ntive. CL. Cherokee County Record (Pryer 1622, Pryer 1641). Crawford County Record (Pryer 1691, Pryer 3506).

Bothriochloa laguroides (DC.) Herter subsp. torreyana (Steud.) Allred \& Gould - Silver Beard Grass. CH [!, K], CRW [!, K, G]. Native. OC, CL.

Bouteloua curtipendula (Michx.) Torr. var. curtipendula - Side-Oats Grama. CH [!, K], CRW [!, K, G]. Native. OP, CL. Gibson did not recognize varieties of this taxon.

Bouteloua dactyloides (Nutt.) Columbus - Buffalo Grass. CH [!], CRW 
[!, K]. Native. OC, CL. Cherokee County Record (Pryer 899, Pryer 3836, Pryer 3952).

Brachyelytrum erectum (Schreb. ex Spreng.) Beauv. - Bearded Shorthusk. CH [!]. Native. Rare. Cherokee County Record (Pryer 5642). Reported in four counties in Kansas; common in Missouri and Arkansas but rare in Oklahoma.

Bromus arvensis L. - Field Brome. CH [!, K], CRW [!, K, G]. Non-native. $\mathrm{OC}, \mathrm{CL}$.

Bromus catharticus Vahl var. catharticus - Rescue Grass. CRW [!]. Non-native. OC. Crawford County Record (Pryer 5987).

Bromus commutatus L. - Bald Brome. CH [!], CRW [!]. Non-native. Kartesz (2019) synomizes this taxon with Bromus racemosus.

Bromus hordeaceus L. subsp. hordeaceus - Soft Brome. CH [!]. Nonnative. OP. Cherokee County Record (Pryer 160, Pryer 5098).

Bromus inermis Leyss. - Smooth Brome. CH [!, K], CRW [!, K, G]. Non-native. OP, OC, CL.

Bromus latiglumis (Scribn. ex Shear) A.S. Hitchc. - Early-Leaf Brome. $\mathrm{CH}[\mathrm{K}]$. Native. Rare. Reported in two counties in Kansas; in Missouri and unreported for Oklahoma or Arkansas.

Bromus pubescens Spreng. - Hairy Woodland Brome. CH [!, K], CRW $[!, K, \mathrm{G}]$. Native. OP, OC.

Bromus racemosus L. - Bald Brome. CH [!, K], CRW [!, K]. Non-native. Noxious. OP, OC, CL.

Bromus secalinus L. - Rye Brome. CH [!, K], CRW [K, G]. Non-native. Noxious. OP, CL.

Bromus squarrosus L. - Corn Brome. CH [!]. Non-native. CL. Cherokee County Record (Pryer 942).

Bromus sterilis L. - Poverty Brome. CH [!], CRW [!]. Non-native. OP, $\mathrm{CL}$. State Record. Cherokee county vouchers (Pryer 416, Pryer 434, Pryer 694, Pryer 701, Pryer 703a). Crawford county vouchers (Pryer 797). Reports of this taxon are scattered throughout the states, with many in Missouri. It has been reported in three counties in Oklahoma and a few scattered throughout Arkansas. The closest reports are from Jasper, Newton, and McDonald counties Missouri.

Bromus tectorum L. - Cheat Grass. CH [!, K], CRW [!, K, G]. Native. $\mathrm{OP}, \mathrm{OC}, \mathrm{CL}$.

Calamagrostis canadensis (Michx.) Beauv. var. canadensis - Bluejoint. CH [!], CRW [!]. Native. CL. Cherokee County Record (Pryer 1817). Crawford County Record (Pryer 2097).

Cenchrus alopecuroides (L.) Thunb. - CRW [Snow 10980]. Non-native. CL. Snow (2017) reported this correctly as a state record but incorrectly as Cenchrus atropururoides (L.) Thunb.

Cenchrus incertus M.A. Curtis - Coastal Sandburr. CRW [G]. Native. Rare. Reported in five counties in Kansas, including this report; common in Oklahoma and Arkansas but rare in Missouri.

Cenchrus longispinus (Hack.) Fernald - Innocent-Weed. CH [K], CRW [G, K]. Native.

Chasmanthium latifolium (Michx.) Yates - Indian Wood-Oats. CH [!, $\mathrm{K}], \mathrm{CRW}[!, \mathrm{K}, \mathrm{G}]$. Native. OP, OC, CL.

Chloris verticillata Nutt. - Tumble Windmill Grass. CH [K], CRW [!, K, $\mathrm{G}$. Native. $\mathrm{OC}, \mathrm{CL}$.

Chloris virgata Sw. - Feather Windmill Grass. CRW [!]. Non-native. CL. Crawford County Record (Pryer 6416).

Cinna arundinacea L. - Sweet Wood-Reed. CH [!, K], CRW [!, K, G]. Native. OP, CL.

Coleataenia anceps (Michx.) Soreng - Beaked Cut-Throat Grass. CH [!, K], CRW [!, K]. Native. OP, OC, CL.

Coleataenia rigidula (Bosc ex Nees) LeBlond subsp. rigidula - RedTop Cut-Throat Grass. CH [!, K], CRW [!, K, G]. Native. CL.

Cortaderia selloana (J.A. \& J.H. Schultes) Aschers. \& Graebn. - Selloa Pampus Grass. Non-native. Cultivated. Not included in numerical tabulations but recorded here for local workers in the future. Pryer 3254). Possibly adventive in Pittsburg, Crawford County, in an abandoned industrial brownfield site (NS., pers. observ.).
Cynodon dactylon (L.) Pers. - Bermuda Grass. CH [!, K], CRW [!, K, $\mathrm{G}]$. Non-native. $\mathrm{CL}$.

Dactylis glomerata L. subsp. glomerata - Orchard Grass. CH [!, K], CRW [!, K, G]. Non-native. OP, OC, CL

Danthonia spicata (L.) Beauv. ex Roemer \& J.A. Schultes - Poverty Wild Oat Grass. CH [!, K], CRW [K]. Native. OP.

Diarrhena americana Beauv. - American beakgrain. CRW [!]. Native. State Record. Voucher (Pryer $1610 b$ [KSP021213]). Known (but rare) in many counties in Missouri, two in eastern Oklahoma, and twelve counties in western and northern Arkansas.

Diarrhena obovata (Gleason) Brandenburg - Hairy Beakgrain. $\mathrm{CH}$ $[!, \mathrm{K}], \mathrm{CRW}[!, \mathrm{K}, \mathrm{G}]$. Native. OP, OC, CL.

Dichanthelium acuminatum (Sw.) Gould \& C.A. Clark var. acuminatum - Tapered Rosette Grass. CH [!, K], CRW [!, K, G]. Native. OP, OC.

Dichanthelium boscii (Poir.) Gould \& C.A. Clark-Bosc's Rosette Grass. $\mathrm{CH}[\mathrm{K}]$. Native. Extirpated.

Dichanthelium clandestinum (L.) Gould-Deer-Tongue Rosette Grass. $\mathrm{CH}[!, \mathrm{K}], \mathrm{CRW}[!, \mathrm{G}]$. Native. OP, OC.

Dichanthelium depauperatum (Muhl.) Gould-Starved Rosette Grass. CRW [!]. Native. CL. Crawford County Record (Pryer 1166).

Dichanthelium dichotomum (L.) Gould var. dichotomum - Cypress Rosette Grass. CH [!], CRW [!]. Native. OP, OC. Cherokee County Record (Pryer 5072, Pryer 5084). Crawford County Record (Pryer 4629, Pryer 6111)

Dichanthelium latifolium (L.) Harville - Broad-Leaf Rosette Grass. $\mathrm{CH}[!, \mathrm{K}]$. Native. OP.

Dichanthelium laxiflorum (Lam.) Gould - Open-Flower Rosette Grass. CH [K]. Native.

Dichanthelium leibergii (Vasey) Freckmann - Leiberg's Rosette Grass. $\mathrm{CH}[\mathrm{K}]$. Native. Rare. Reported from six counties in Kansas; rare in Missouri and not yet reported for Oklahoma or Arkansas.

Dichanthelium linearifolium (Scribn. ex Nash) Gould - Slim-Leaf Rosette Grass. CH [!, K], CRW [!, K]. Native. OP, CL.

Dichanthelium malacophyllum (Nash) Gould - Soft-Leaf Rosette Grass. CH [!, K], CRW [!]. Native. CL. Crawford County Record (Pryer 1443)

Dichanthelium oligosanthes (J.A. Schultes) Gould - Heller's Rosette Grass. CH [K], CRW [!, K, G]. Native. OC, CL.

Dichanthelium scoparium (Lam.) Gould - Broom Rosette Grass. CH $[!, \mathrm{K}]$, CRW $[!, \mathrm{G}]$. Native. OP, OC.

Dichanthelium sphaerocarpon (Elliott) Gould - Round-Seed Rosette Grass. CH [!, K], CRW [!, K, G]. Native. OP, OC, CL. Kartesz (2017) recognizes the varieties isophyllum and sphaerocarpon, however due to the difficulty in identifying this genus, no specimens were identified to the infraspecific level.

Digitaria ciliaris (Retz.) Koel. - Southern Crab Grass. CH [!, K], CRW [!, K]. Non-native. OP, OC, CL.

Digitaria cognata (J.A. Schultes) Pilger - Carolina Crab Grass. CRW $[!, \mathrm{G}]$. Native. CL.

Digitaria filiformis (L.) Koel. var. filiformis - Slender Crab Grass. CH [K], CRW [K, G]. Native.

Digitaria ischaemum (Schreb. ex Schweig.) Schreb. ex Muhl. Smooth Crab Grass. CH [!, K], CRW [!, K]. Non-native. OP, OC, CL.

Digitaria sanguinalis (L.) Scop. - Hairy Crab Grass. CH [!, K], CRW [!, $\mathrm{K}, \mathrm{G}]$. Non-native. OP, OC, CL.

Dinebra panicea (Retz.) P.M. Peterson \& N. Snow subsp. brachiata (Steudl.) P.M. Peterson \& N. Snow - Needle Viper Grass. CH [!], CRW [!, K]. Native. CL. Cherokee County Record (Pryer 2490).

Dinebra panicea (Retz.) P.M. Peterson \& N. Snow subsp. mucronata (Michx.) P.M. Peterson \& N. Snow - Needle Viper Grass. CH [K], CRW [K]. Native. 
Diplachne fusca (L.) Beauv. ex Roemer \& J.A. Schultes subsp. fascicularis (Lam.) P.M. Peterson \& N. Snow - Bearded Beetle Grass. CRW [!, K]. Native. CL.

Echinochloa colona (L.) Link - Jungle-Rice. CRW [!]. Non-native. CL. Crawford County Record (Pryer 3563).

Echinochloa crus-galli (L.) Beauv. - Large Barnyard Grass. CH [!], CRW [!]. Non-native. OC, CL. Cherokee County Record (Pryer 3767, Pryer 5470). Crawford County Record (Pryer 1363b, Pryer 1364, Pryer 1407, Pryer 1496, Pryer 1555, Pryer 1999, Pryer 2667, Pryer 6038).

Echinochloa muricata (Beauv.) Fernald var. microstachya Wieg. - Rough Barnyard Grass. CH [!, K], CRW [!]. Native. OC, CL. Crawford County Record (Pryer 1407b, Pryer 1496b, Pryer 1689, Pryer 2811, Pryer 5493).

Echinochloa muricata (Beauv.) Fernald var. muricata - Rough Barnyard Grass. CH [!, K], CRW [!, K, G]. Native. OP, OC, CL.

Eleusine indica (L.) Gaertn. - Indian Goose Grass. CH [!, K], CRW [!, K, G]. Non-native. OC, CL.

Elymus canadensis L. - Nodding Wild Rye. CH [!, K], CRW [!, K, G]. Native. OC, CL.

Elymus glabriflorus (Vasey) Scribn. \& Ball - Southeastern Wild Rye. CH [!, K], CRW [!]. Native. OP, OC, CL. Crawford County Record (Pryer 826, Pryer 995, Pryer 996, Pryer 1049, Pryer 1340, Pryer 1448, Pryer 1528, Pryer 1543, Pryer 1692, Pryer 2595). Many references previously did not include this taxon as an option in the key, having it subsumed under other taxa.

Elymus hystrix L. var. hystrix - Eastern Bottle-Brush Grass. CH [K]. Native.

Elymus macgregorii R.E. Brooks \& J. Campbell - Macgregor's Wild Rye. $\mathrm{CH}[\mathrm{K}]$. Native.

Elymus $\times$ maltei Bowden $-\mathrm{CH}[\mathrm{K}]$. Native.

Elymus repens (L.) Gould - Creeping Wild Rye. CH [!], CRW [!]. Nonnative. Noxious. CL. Cherokee County Record (Pryer 944). Crawford County Record (Pryer 822).

Elymus riparius Wieg. - River-Bank Wild Rye. CH [!], CRW [!]. Native. OP, OC, CL. State Record. Cherokee county vouchers (Pryer 911, Pryer 1727). Crawford county voucher (Pryer 5198). This taxon has mostly an eastern distribution, with scattered reports in Missouri and Arkansas; not reported for Oklahoma, and with closest reports from Barton, Jasper, and McDonald counties Missouri.

Elymus villosus Muhl. ex Willd. - Hairy Wild Rye. CH [!, K], CRW [!]. Native. OP, OC, CL. Crawford County Record (Pryer 1329, Pryer 2072, Pryer 2112).

Elymus virginicus L. var. virginicus - Virginia Wild Rye. CH [!, K], CRW $[!, \mathrm{K}, \mathrm{G}]$. Native. OP, OC, CL.

Eragrostis capillaris (L.) Nees - Lace Grass. CH [!, K], CRW [K, G]. Native. CL.

Eragrostis cilianensis (All.) Vignolo ex Janch. - Stink Grass. CH [!, K], CRW [!, K, G]. Non-native. OC, CL.

Eragrostis frankii C.A. Mey. ex Steud. - Sandbar Love Grass. CH [!], CRW [!]. Native. OP, CL. Cherokee County Record (Pryer 2305, Pryer 3004, Pryer 3732). Crawford County Record (Pryer 2902).

Eragrostis hypnoides (Lam.) B.S.P. - Teal Love Grass. CH [K], CRW [K]. Native.

Eragrostis intermedia A.S. Hitchc. - Plains Love Grass. CH [!, K], CRW [!, K]. Native. OP, OC, CL.

Eragrostis minor Host - Little Love Grass. CRW [!, G]. Non-native. OC. Eragrostis pectinacea (Michx.) Nees ex Jedw. var. pectinacea - Purple Love Grass. CH [!, K], CRW [!, K, G]. Native. OC, CL. Gibson did not recognize varieties of this taxon.

Eragrostis pilosa (L.) Beauv. var. pilosa - Indian Love Grass. CRW [!]. Native. Adventive. OC. State Record (Pryer 5452).

Eragrostis secundiflora J. Presl subsp. oxylepis (Torr.) S.D. Koch -
Red Love Grass. CH [!]. Native. CL. Cherokee County Record (Pryer 1573).

Eragrostis spectabilis (Pursh) Steud. - Petticoat-Climber. CH [!, K], CRW [!, K, G]. Native. OC, CL.

Eragrostis trichodes (Nutt.) Wood - Sand Love Grass. CRW [K, G]. Native.

Eriochloa contracta A.S. Hitchc. - Prairie Cup Grass. CH [K], CRW [!, $\mathrm{K}, \mathrm{G}]$. Native. OC.

Festuca paradoxa Desv. - Clustered Fescue. CH [K], CRW [K]. Native. Festuca rubra L. subsp. rubra - Red Fescue. CH [!], CRW [!, K, G]. Native. Adventive. OP, OC, CL. Cherokee County Record (Pryer 1121, Pryer 1226, Pryer 5010, Pryer 5080, Pryer 5375, Pryer 5416).

Festuca subverticillata (Pers.) Alexeev - Nodding Fescue. CH [!, K], CRW [!, K]. Native. OC, CL.

Glyceria striata (Lam.) A.S. Hitchc. - Fowl Manna Grass. CH [K], CRW [!, K, G]. Native. OC, CL.

Holcus lanatus L. - Common Velvet Grass. CH [K], CRW [K]. Non-native.

Hordeum jubatum L. subsp. jubatum - Fox-Tail Barley. CH [!, K], CRW $[!, K, G]$. Native. CL. Gibsons did not recognize subspecies of this taxon.

Hordeum pusillum Nutt. - Little Barley. CH [!, K], CRW [!, K, G]. Native. $\mathrm{OP}, \mathrm{OC}, \mathrm{CL}$.

Koeleria macrantha (Ledeb.) J.A. Schultes - Prairie Koeler's Grass. $\mathrm{CH}[!, \mathrm{K}], \mathrm{CRW}[!, \mathrm{K}, \mathrm{G}]$. Native. OC, CL.

Leersia lenticularis Michx. - Catchfly Grass. CH [K]. Native. Rare. Reported in four counties in Kansas but common in surrounding states.

Leersia oryzoides (L.) Sw. - Rice Cut Grass. CH [!, K], CRW [!, K]. Native. $\mathrm{OP}, \mathrm{CL}$.

Leersia virginica Willd. - White Grass. CH [!, K], CRW [!, K]. Native. $\mathrm{OC}, \mathrm{CL}$.

Lolium perenne L. subsp. multiflorum (Lam.) Husnot - Perennial Rye Grass. CH [!], CRW [!, G]. Non-native. OC, CL. Cherokee County Record (Pryer 936).

Lolium perenne L. subsp. perenne - Perennial Rye Grass. CH [!, K], CRW [!, G]. Non-native. OP, CL.

Lolium temulentum L. subsp. temulentum - Poison Darnel. CH [K]. Non-native.

Melica nitens (Scribn.) Nutt. ex Piper - Three-Flower Melic Grass. CH $[!, K], C R W[K, G]$. Native. Rare. OP. Reported in sixteen counties in Kansas but common in surrounding states.

Microstegium vimineum (Trin.) A. Camus - Japanese Stilt Grass. CH [!]. Non-native. OP. State Record. Cherokee county voucher (Pryer 6318). Reported throughout the eastern United States including scattered reports in Missouri, Arkansas, and Oklahoma. The closest reports are from McDonald and Barry counties Missouri and Delaware County, Oklahoma.

Muhlenbergia bushii Pohl - Nodding Muhly. CH [!, K], CRW [!]. Native. OP, OC. Crawford County Record (Pryer 5962, Pryer 5969, Pryer 6007, Pryer 6067, Pryer 6205, Pryer 6255).

Muhlenbergia capillaris (Lam.) Trin. - Hair-Awn Muhly. CH [K]. Native.

Muhlenbergia cuspidata (Torr. ex Hook.) Rydb. - Stony-Hills Muhly. CH [!], CRW [!]. Native. OP, OC, CL. Cherokee County Record (Pryer 6329). Crawford County Record (Pryer 6195, Pryer 6253).

Muhlenbergia frondosa (Poir.) Fernald - Wire-Stem Muhly. CH [!, K], CRW [!, K]. Native. OC, CL.

Muhlenbergia mexicana (L.) Trin. - Mexican Muhly. CH [K], CRW [G]. Native.

Muhlenbergia paniculata (Nutt.) Columbus - Tumble Grass. CH [K], CRW [K, G]. Native.

Muhlenbergia racemosa (Michx.) B.S.P. - Green Muhly. CRW [!]. Native. CL. Crawford County Record (Pryer 3268, Pryer 3368).

Muhlenbergia schreberi J.F. Gmel. - Nimblewill. CH [!, K], CRW [!, K, G]. Native. OP, OC. 
Muhlenbergia sobolifera (Muhl. ex Willd.) Trin. - Rock Muhly. CH [!, $\mathrm{K}]$. CRW [!, K]. Native. OP, OC, CL.

Muhlenbergia sylvatica (Torr.) Torr. ex A. Gray - Woodland Muhly. $\mathrm{CH}[\mathrm{K}]$.

Neeragrostis reptans (Michx.) Nicora - Creeping Love Grass. $\mathrm{CH}$ [K]. Native.

Panicum capillare L. - Common Panic Grass. CH [!, K], CRW [!, K, G]. Native. OP, OC, CL.

Panicum dichotomiflorum Michx. var. dichotomiflorum - Fall Panic Grass. CH [!, K], CRW [!, K, G]. Native. OP, OC, CL. Gibson did not recognize varieties of this taxon.

Panicum flexile (Gattinger) Scribn. - Wiry Panic Grass. CH [!, K], CRW [K]. Native. OP.

Panicum philadelphicum Bernh. ex Trin. - Philadelphia Panic Grass. $\mathrm{CH}[!, \mathrm{K}], \mathrm{CRW}[!, \mathrm{K}]$. Native. OP, OC, CL.

Panicum virgatum L. var. virgatum - Wand Panic Grass. CH [!, K], CRW [!, K, G]. Native. CL. Gibson did not recognize varieties of this taxon.

Pascopyrum smithii (Rydb.) A. Löve - Western-Wheat Grass. CH [K], CRW [!, K, G]. Native. OC, CL.

Paspalum dilatatum Poir. - Golden Crown Grass. State Record. Cherokee County (Curran s.n., Oct 2017; confirmed by N. Snow).

Paspalum floridanum Michx. - Florida Crown Grass. CH [!, K], CRW $[!, \mathrm{K}, \mathrm{G}]$. Native. OC, CL.

Paspalum laeve Michx. - Field Crown Grass. CH [!, K], CRW [!, K, G]. Native. $\mathrm{OP}, \mathrm{OC}, \mathrm{CL}$.

Paspalum pubiflorum Rupr. ex Fourn. - Hairy-Seed Crown Grass. $\mathrm{OC}, \mathrm{CL}$.

Paspalum repens Berg. var. fluitans (Elliott) J. Wipff \& S.D. Jones Horse-Tail Crown Grass. CH [K]. Native.

Paspalum setaceum Michx. - Slender Crown Grass. CH [!, K], CRW $[!, \mathrm{K}, \mathrm{G}]$. Native. OC, CL.

Phalaris arundinacea L. - Reed Canary Grass. CH [!]. CRW [!]. Native. CL. Cherokee County Record (Pryer 520, Pryer 632). Crawford County Record (Pryer 1953, Pryer 2127).

Phalaris caroliniana Walt. - May Grass. CRW [K, G]. Native.

Phleum pratense L. - Common Timothy. CH [!, K], CRW [!, K, G]. Non-native. OP, OC, $\mathrm{CL}$.

Phragmites australis (Cav.) Trin. ex Steud. subsp. americanus Saltonst, P.M. Peterson, \& Soreng - Common Reed. CRW [!]. Native. CL. Crawford County Record (Pryer 3254).

Phragmites australis (Cav.) Trin. ex Steud. subsp. australis - Common Reed. CRW [!, K]. Non-native. CL.

Poa annua L. - Annual Blue Grass. CH [!, K], CRW [!, K, G]. Nonnative. CL.

Poa bulbosa L. subsp. vivipara (Koel.) Arcang. - Bulbous Blue Grass. $\mathrm{CH}[\mathrm{K}]$. Non-native.

Poa chapmaniana Scribn. - Chapman's Blue Grass. CH [!, K], CRW [!]. Native. Rare. OP, CL. Crawford County Record (Pryer 29, Pryer 66, Pryer 4063, Pryer 4300). Reported in nine counties in Kansas, including this report; common in Missouri and Arkansas, but rare and with a questionable presence in Oklahoma.

Poa compressa L. - Flat-Stem Blue Grass. CH [!K], CRW [!, K, G]. Non-native. OC, CL.

Poa pratensis L. subsp. pratensis - Kentucky Blue Grass. CH [!, K], CRW [!, K, G]. Non-native. OC, CL. Gibson did not recognize subspecies of this taxon.

Poa sylvestris A. Gray - Woodland Blue Grass. CH [K], CRW [!, K, G]. Native. CL.

Schedonorus arundinaceus (Schreb.) Dumort. - Tall False Rye Grass. $\mathrm{CH}[!, \mathrm{K}], \mathrm{CRW}[!, \mathrm{K}]$. Non-native. OP, OC, CL.

Schedonorus pratensis (Huds.) Beauv. - Meadow False Rye Grass. $\mathrm{CH}$ [!, K], CRW [!, K, G]. Non-native. OC, CL.

Schizachyrium scoparium (Michx.) Nash var. scoparium - Little False Bluestem. CH [!, K], CRW [!, K, G]. Native. OP, CL.
Sclerochloa dura (L.) Beauv. - Common Hard Grass. CRW [K]. Non-native.

Setaria faberi R.A.W. Herrm. - Japanese Bristle Grass. CH [!, K], CRW [!, K]. Non-native. Noxious. OP, CL.

Setaria italica (L.) Beauv. - Italian Bristle Grass. CH [!]. Non-native. CL. Cherokee County Record (Pryer 1825).

Setaria parviflora (Poir.) Kerguélen - Marsh Bristle Grass. CH [!, K], CRW [!, K, G]. Native. OP, OC, CL.

Setaria pumila (Poir.) Roemer \& J.A. Schultes subsp. pumila - Yellow Bristle Grass. CH [!, K], CRW [!, K]. Non-native. OC, CL.

Setaria viridis (L.) Beauv. var. viridis - Green Bristle Grass. CH [!, K], CRW [!, K, G]. Non-native. OP, OC, CL. Gibson did not recognize varities of this taxon.

Sorghastrum nutans (L.) Nash - Yellow Indian Grass. CH [!, K], CRW [!, K, G]. Native. CL.

Sorghum bicolor (L.) Moench subsp. bicolor - Broom-Corn. CH [K], CRW [K, G]. Non-native.

Sorghum bicolor (L.) Moench subsp. drummondii (Steud.) de Wet ex Davidse - Broom-Corn. CH [!], CRW [!]. Non-native. CL. State Record. Cherokee County Vouchers (Pryer 5823). This subspecies has scattered reports in several states including Missouri; unreported for Oklahoma or Arkansas with the closest reports from Jasper and McDonald counties, Missouri. Previous concepts of infraspecific taxa have varied. Mark Mayfield (KSC, pers. comm. 2018) indicates that Gates (1936) likely saw some specimens for Kansas but reported them as S. bicolor var. sudanense (Piper) A.S. Hitchc. Thus, this is the first report of this taxon for Kansas under this name.

Sorghum halepense (L.) Pers. - Johnson Grass. CH [!, K], CRW [!, K, G]. Non-native. Noxious. OP, OC, CL.

Sphenopholis intermedia (Rydb.) Rydb. - Slender Wedgescale. $\mathrm{CH}$ [K], CRW [!, K, G]. Native. CL.

Sphenopholis obtusata (Michx.) Scribn. - Prairie Wedgescale. CH [!, $\mathrm{K}], \mathrm{CRW}[!, \mathrm{K}, \mathrm{G}]$. Native. OC, CL.

Sporobolus clandestinus (Biehler) A.S. Hitchc. - Rough Dropseed. $\mathrm{CH}[!, \mathrm{K}], \mathrm{CRW}[!, \mathrm{K}]$. Native. OC, CL.

Sporobolus compositus (Poir.) Merr. var. compositus - Head-Like Dropseed. CH [!, K], CRW [!, K, G]. Native. OC, CL.

Sporobolus compositus (Poir.) Merr. var. macer (Trin.) Kartesz \& Gandhi - Head-Like Dropseed. CH [K], CRW [!]. Native. OC, CL. Crawford County Record (Pryer 3376, Pryer 5690, Pryer 6280).

Sporobolus michauxianus (A.S. Hitchc.) P.M. Peterson \& Saarela - Freshwater Cord Grass. CH [!, K], CRW [!, K, G]. Native. CL. (Synonym: Spartina pectinata Bosc ex Link).

Sporobolus neglectus Nash - Small Dropseed. CH [!, K], CRW [!, K, G]. Native. OC, CL.

Sporobolus pyramidatus (Lam.) A.S. Hitchc - Target Dropseed. CRW [!, K]. Native. CL.

Sporobolus vaginiflorus (Torr. ex A. Gray) Wood - Poverty Dropseed. $\mathrm{CH}[\mathrm{K}], \mathrm{CRW}[$ !, K]. Native. CL.

Thinopyrum intermedium (Host) Barkworth \& D.R. Dewey Intermediate Quack Grass. CRW [!]. Non-native. CL. Crawford County Record (Pryer 1373).

Tridens flavus (L.) A.S. Hitchc. var. flavus - Tall Redtop. CH [!, K], CRW [!, K, G]. Native. OP, OC, CL.

Tridens muticus (Torr.) var. elongatus (Buckl.) Shinners - NashAwnless Fluff Grass. CH [K], CRW [K]. Native.

Tridens strictus (Nutt.) Nash - Long-Spike Fluff Grass. CH [!, K], CRW [!, K, G]. Native. OP, OC, CL.

Tripsacum dactyloides (L.) L - Eastern Mock Grama. CH [!, K], CRW $[!, \mathrm{K}, \mathrm{G}]$. Native. OP, CL.

Triticum aestivum L - Bread Wheat. CH [!], CRW [!]. Non-native. OP, OC, CL. Cherokee County Record (Pryer 635, Pryer 934, Pryer 5121). Crawford County Record (Pryer 468, Pryer 794, Pryer 800 ). 
Urochloa platyphylla (Munro ex Wright) R. Webster - Broad-Leaf Liverseed Grass. CH [!]. Native. CL. State Record (Pryer 5536).

Vulpia myuros (L.) K.C. Gmel - Rat-Tail Six-Weeks Grass. CH [!, K], CRW [K]. Non-native. OP.

Vulpia octoflora (Walt.) Rydb. var. octoflora - Eight-Flower Six-Weeks Grass. CH [!, K], CRW [!, K, G]. Native. OP, CL. Gibson did not recognize varieties of this taxon.

POLEMONIACEAE (Phlox Family)

Phlox divaricata L - Wild Blue Phlox. CH [!, K], CRW [!, K, G]. Native. $\mathrm{OP}, \mathrm{OC}, \mathrm{CL}$.

Phlox paniculata L - Fall Phlox. CH [K]. Native.

Phlox pilosa L - Downy Phlox CRW [G]. Gibson report. Gibson did not recognize subspecies of this taxon.

Phlox pilosa L. subsp. ozarkana (Wherry) Wherry - Downy Phlox. $\mathrm{CH}[\mathrm{K}]$. Native.

Phlox pilosa L. subsp. pilosa - Downy Phlox. CH [!, K], CRW [!, K]. Native. OP, OC.

Polemonium reptans L. var. reptans - Greek-Valerian. $\mathrm{CH}$ [K]. Native. Rare. Reported in four counties in Kansas but common in surrounding states.

POLYGALACEAE (Milkwort Family)

Polygala incarnata L - Procession-Flower. $\mathrm{CH}[\mathrm{K}], \mathrm{CRW}[\mathrm{K}, \mathrm{G}]$. Native.

Polygala sanguinea L - Purple Milkwort. CH [!, K], CRW [!, K, G]. Native. $\mathrm{OP}, \mathrm{CL}$.

Polygala senega L - Seneca-Snakeroot. CH [K]. Native. Rare. Only reported in Cherokee County, Kansas; common in Missouri and Arkansas but rare in Oklahoma.

Polygala verticillata $\mathrm{L}$ - Whorled Milkwort. CH [K]. Native.

POLYGONACEAE (Buckwheat Family)

Fallopia convolvulus (L.) A. Löve - Black-Bindweed. CH [K], CRW [!, $\mathrm{K}, \mathrm{G}]$. Non-native. Noxious. CL.

Fallopia scandens (L.) Holub - Climbing Black-Bindweed. CH [!, K], CRW [!, K, G]. Native. OP, CL.

Persicaria amphibia (L.) S.F. Gray p.p - Water Smartweed. CH [!, K], CRW [!, K, G]. Native. OC, CL.

Persicaria bicornis (Raf.) Nieuwl - Pink Knotweed. CH [!, K], CRW $[\mathrm{K}, \mathrm{G}]$. Native. CL.

Persicaria hydropiper (L.) Delarbre - Mild Water-Pepper. CH [!], CRW [!, K, G]. Non-native. OP, CL. Cherokee County Record (Pryer 2482, Pryer 5368).

Persicaria hydropiperoides (Michx.) Small - Swamp Smartweed. CH [!, K], CRW [!, K, G]. Native. OP, CL.

Persicaria lapathifolia (L.) S.F. Gray - Dock-Leaf Smartweed. CH [!, $\mathrm{K}], \mathrm{CRW}[\mathrm{K}, \mathrm{G}]$. Native. CL.

Persicaria longiseta (Bruijn) Kitagawa - Bristly Lady's-Thumb. CH [!], CRW [!]. Non-native. OC. Crawford County Record (Pryer 1993, Pryer 3328, Pryer 3454, Pryer 6024, Pryer 6142, Pryer 6176, Snow 11121). Cherokee County Record (Pryer 5797)

Persicaria maculosa S.F. Gray - Spotted Lady's-Thumb. CH [!, K], CRW $[!, \mathrm{K}, \mathrm{G}]$. Non-native. OP, OC, CL.

Persicaria pensylvanica (L.) M. Gómez - Pinkweed. CH [!, K], CRW [!, $\mathrm{K}, \mathrm{G}]$. Native. OP, $\mathrm{CL}$.

Persicaria punctata (Elliott) Small - Dotted Smartweed. CH [!, K], CRW [!, K, G]. Native. OP, OC, CL.

Persicaria sagittata (L.) H. Gross - Arrow-Leaf Tearthumb. CRW [G]. Native. Rare. Reported in three counties in Kansas but common in surrounding states.

Persicaria virginiana (L.) Gaertn. - Jumpseed. CH [!, K], CRW [!, K, G]. Native. $\mathrm{OP}, \mathrm{OC}, \mathrm{CL}$.

Polygonum aviculare L. subsp. aviculare - Yard Knotweed. CH [K], CRW [!, K]. Non-native. CL.

Polygonum aviculare L. subsp. depressum (Meisn.) Arcang. - Yard Knotweed. CH [K], CRW [!, K, G]. Non-native. OC, CL.
Polygonum ramosissimum Michx. - Yellow-Flower Knotweed. CRW [G]. Gibson did not recognize subspecies of this taxon.

Polygonum ramosissimum Michx. subsp. prolificum (Small) Costea \& Tardif - Yellow-Flower Knotweed. CRW [K]. Native.

Polygonum ramosissimum Michx. subsp. ramosissimum - YellowFlower Knotweed. CH [!, K], CRW [!, K]. Native. Non-native. Noxious. OP, CL.

Reynoutria japonica Houtt. - Japanese-Knotweed. CRW [K]. Non-native.

Rumex acetosella L. - Common Sheep Sorrel. CH [!, K], CRW [!, K, G]. Non-native. Noxious. OP, CL.

Rumex altissimus Wood - Pale Dock. CH [!, K], CRW [!, K, G]. Native. Noxious. OC, CL.

Rumex crispus L. subsp. crispus - Curly Dock. CH [!, K], CRW [!, K, G]. Non-native. Noxious. OP, OC, CL. Gibson did not recognize subspecies of this taxon.

Rumex hastatulus Baldw. - Heart-Wing Sorrel. CH [K]. Native. Extirpated. The most recent specimen was made on June 9, 2002 (Holland 10154B) for Woodson County, Kansas. This specimen resides at McGregor Herbarium, Univsity of Kansas.

Rumex obtusifolius L. - Bitter Dock. CH [!, K]. Non-native. Noxious. $\mathrm{OP}, \mathrm{CL}$.

Rumex patientia L. - Patience Dock. CH [K]. Non-native. Noxious.

Rumex pulcher L. - Fiddle Dock. CH [!], CRW [G]. Non-native. Noxious. OP. Cherokee County Record (Pryer 1716).

Rumex verticillatus L. - Swamp Dock. CH [K]. Native. Rare. Reported in eight counties in Kansas; common in Missouri and Arkansas where it is considered noxious, but rare in Oklahoma.

PONTEDERIACEAE (Pickerelweed Family)

Heteranthera limosa (Sw.) Willd. - Blue Mud-Plantain. CH [K]. Native.

Pontederia cordata L. - Pickerelweed. CH [K]. Rare. Reported in two counties in Kansas; common in Missouri and Arkansas but rare in Oklahoma.

PORTULACACEAE (Purslane Family)

Portulaca oleracea L. - Little-Hogweed. CH [K], CRW [!, K, G]. Native. Adventive. OC.

Portulaca pilosa L. - Kiss-Me-Quick. CRW [!, K, G]. Non-native. OC.

POTAMOgetonACEAE (Pondweed Family)

Potamogeton crispus L. - Curly Pondweed. CH [K], CRW [!, K, G]. Non-native. CL.

Potamogeton diversifolius Raf. - Waterthread. CH [K], CRW [!, K, G]. Native. CL.

Potamogeton foliosus Raf. - Leafy Pondweed. CH [K], CRW [K, G]. Native.

Potamogeton nodosus Poir. - Long-Leaf Pondweed. CH [K], CRW [!, $\mathrm{K}, \mathrm{G}]$. Native. OC, CL.

Potamogeton pusillus L. - Small Pondweed. CRW [!, K]. Native. CL.

Stuckenia pectinata (L.) Böerner - Sago False Pondweed. CRW [K, G]. Native.

Zannichellia palustris L. - Horned-Pondweed. CRW [!]. Native. OC. Crawford County Record (Pryer 5621).

PRIMULACEAE (Primrose Family)

Androsace occidentalis Pursh - Western Rock-Jasmine. $\mathrm{CH}$ [K], CRW [!, K, G]. Native. OC, CL.

Lysimachia ciliata L. - Fringed Yellow-Loosestrife. CH [!, K], CRW [K, G]. Native. CL.

Lysimachia minima (L.) U. Manns \& A. Anderb. - Chaffweed. CH [!, $\mathrm{K}]$, CRW [!]. Native. OP, CL.

Lysimachia nummularia L. - Creeping-Jenny. CH [K]. Non-native.

Primula meadia (L.) A.R. Mast \& Reveal - Pride-of-Ohio. CH [K], CRW $[!, K, G]$. Native. OC.

Samolus parviflorus Raf. - Water-Pimpernel. CH [K]. Native. 
RANUNCULACEAE (Buttercup Family)

Anemonastrum canadense (L.) Mosyakin - Round-LeafThimbleweed. CH [!], CRW [!]. Native. OP, CL. Cherokee County Record (Pryer 1173). Crawford County Record (Pryer 1357). In Kansas known from twelve counties, mostly in the northeast; more common mostly north.

Anemone caroliniana Walt. - Carolina Thimbleweed. CH [K], CRW [K, G]. Native.

Anemone virginiana L. var. virginiana - Tall Thimbleweed. $\mathrm{CH}[\mathrm{K}]$, CRW [K, G]. Native.

Aquilegia canadensis L. - Red Columbine. CH [!, K], CRW [!, K, G]. Native. OP, OC.

Clematis catesbyana Pursh - Satincurls. CH [K]. Native. Rare. Only reported in Cherokee County, Kansas; common in a small region in Missouri and Arkansas and considered extirpated in Oklahoma.

Clematis pitcheri Torr. \& A. Gray var. pitcheri-Bluebill. CH [!, K], CRW [!, K, G]. Native. OP, CL. Gibson did not recognize varieties of this taxon.

Clematis terniflora DC. - Sweet Autumn Virgin's-Bower. CH [!, K], CRW [!, K, G]. Non-native. OP, CL.

Delphinium carolinianum Walt. - Carolina Larkspur. CRW [G]. Gibson did not recognize subspecies of this taxon.

Delphinium carolinianum Walt. subsp. carolinianum - Carolina Larkspur. CH [K], CRW [!]. Native. OC, CL. Crawford County Record (Pryer 855, Pryer 4887).

Delphinium carolinianum Walt. subsp. virescens (Nutt.) R.E. Brooks - Carolina Larkspur. CH [K], CRW [!, K]. Native. CL.

Delphinium tricorne Michx. - Dwarf Larkspur. CH [!, K], CRW [K, G]. Native. OP.

Enemion biternatum Raf. - Eastern False Rue-Anemone. CH [!, K], CRW [K, G]. Native. OP.

Ficaria verna Huds. subsp. calthifolia (Rchb.) Nyman - EurasianButtercup. CH [!]. Non-native. CL. State Record. Cherokee county voucher (Pryer 6421). Abundant on a private property, including its wooded areas. This taxon has been reported in few counties nationwide and mostly in northern states with the exception of Alabama. The closest report is from St. Louis County, Missouri; not reported for Oklahoma or Arkansas.

Myosurus minimus L. - Tiny Mousetail. CH [K], CRW [K, G]. Native.

Ranunculus abortivus L. - Kidney-Leaf Buttercup. CH [!, K], CRW [!, $\mathrm{K}, \mathrm{G}$. Native. $\mathrm{OC}, \mathrm{CL}$.

Ranunculus acris L. var. acris - Tall Buttercup. CRW [!]. Non-native. OC. Crawford County Record (Pryer 4476).

Ranunculus bulbosus L. - St. Anthony's-Turnip. CH [K]. Non-native. Ranunculus fascicularis Muhl. ex Bigelow - Early Buttercup. CH [!, $\mathrm{K}], \mathrm{CRW}[\mathrm{K}, \mathrm{G}]$. Native. OP.

Ranunculus hispidus Michx. var. hispidus - Bristly Buttercup. CH [!, K]. Native. Rare. OP. Reported in two counties in Kansas but common in surrounding areas.

Ranunculus hispidus Michx. var. nitidus (Chapman) T. Duncan - Bristly Buttercup. CRW [K]. Native. Rare. Reported in ten counties in Kansas but common in surrounding states.

Ranunculus laxicaulis (Torr. \& A. Gray) Darby-Mississippi Buttercup. $\mathrm{CH}[\mathrm{K}]$. Native. Rare. Reported in two counties in Kansas but common in surrounding states.

Ranunculus longirostris Godr. - Long-Beak Water-Crowfoot. CRW [!]. Native. CL. County Record (Snow 11101). Abundant in one of the strip pits in Mined Land Wildlife Area No. 1, north of Pittsburg.

Ranunculus micranthus Nutt. - Rock Buttercup. CH [!, K], CRW [!, K, G]. Native. OP, OC, CL.

Ranunculus recurvatus Poir. var. recurvatus - Blisterwort. CH [!, K], CRW [!]. Native. CL. Rare. Crawford county record (Pryer 353).
Reported in seven counties in Kansas, including this report, but common in surrounding states.

Ranunculus repens L. - Creeping Buttercup. CH [!], CRW [!]. Nonnative. OP, OC. Cherokee County Record (Pryer 4525). Crawford County Record (Pryer 4215).

Ranunculus sardous Crantz - Hairy Buttercup. CH [!], CRW [!, K]. Nonnative. OC, CL. Cherokee County Record (Pryer 488, Pryer 929, Pryer 1710, Pryer 4202).

Ranunculus sceleratus L. var. sceleratus - Cursed Buttercup. CH [!, K], CRW [!, K]. Native. OC, CL.

Thalictrum dasycarpum Fisch. \& Avé-Lall. - Purple Meadow-Rue. CH $[!, \mathrm{K}]$, CRW [!, K, G]. Native. OP, CL.

Thalictrum dioicum L. - Early Meadow-Rue. CH [!]. Native. OP. Cherokee County Record (Pryer 432). Was considered extirpated from the state.

Thalictrum thalictroides (L.) Eames \& B. Boivin - Rue-Anemone. $\mathrm{CH}$ $[!, \mathrm{K}]$. CRW [G]. Native. OP.

RHAMNACEAE (Buckthorn Family)

Ceanothus americanus L. - New Jersey-Tea. CH [!, K], CRW [G]. Native. OP.

Ceanothus herbaceus Raf. - Prairie Redroot. CH [K], CRW [K, G]. Native.

Rhamnus japonica Maxim. - Japanese Buckthorn. CRW [G]. Nonnative. Gibson (1963) collected a specimen (Gibson s.n., 22 Sep 1962 [KSP014101]) at Pittsburg State University's Natural History Reserve but identified it as Rhamnus lanceolata Pursh. The Reserve is a large area of reclaimed suface mining, and the one specimen almost assuredly was planted. Only reported in three counties nationwide, including two in Missouri and one in Illinois; the closest reports are from Jackson and Boone counties Missouri. Information provided here for local botanists, but not included among numerical tallies.

Rhamnus lanceolata Pursh subsp. glabrata (Gleason) Kartesz \& Gandhi-Lance-Leaf Buckthorn. CH [K], CRW [!, K, G]. Native. OC.

ROSACEAE (Rose Family)

Agrimonia gryposepala Wallr. - Tall Hairy Grooveburr. CH [!]. Native. Rare. OP. Cheroke County Record (Pryer 1733). Reported in two counties in Kansas and rare in Missouri and Arkansas. This taxon has not been reported in the state of Oklahoma.

Agrimonia parviflora Aiton - Harvestlice. CH [K], CRW [G]. Native.

Agrimonia pubescens Wallr. - Soft Grooveburr. CH [!, K], CRW [!, G]. Native. Crawford County Record (Pryer 2004, Pryer 6159, Pryer 6271). OP, OC, CL.

Agrimonia rostellata Wallr. - Beaked Grooveburr. CH [!, K]. Native. Rare. OP. Reported in seven counties in Kansas but common in surrounding states.

Amelanchier arborea (Michx. f.) Fernald - Downy Service-Berry. CH $[!, \mathrm{K}], \mathrm{CRW}[\mathrm{K}]$. Native. OP.

Chaenomeles speciosa (Sweet) Nakai-Common Flowering-Quince. $\mathrm{CH}$ [!]. Non-native. Probably a persisting planting at former homestead. Included here for future reference, but not included in numerical tabulations.

Crataegus berberifolia Torr. \& A. Gray - Barberry Hawthorn. CH [K]. Native.

Crataegus calpodendron (Ehrh.) Medik. - Pear Hawthorn. CH [K], CRW [Hess 53 [KSP024589]. Native. Crawford County Record. Rare. CL. In Kansas known only form Cherokee and Crawford counties; more common mostly east.

Crataegus coccinioides Ashe - Kansas Hawthorn. CH [K]. Native. Extirpated. The most recent specimen was made on August 2, 1956 (E.W. Lathrop 2713) for Cherokee County, Kansas. This specimen resides at McGregor Herbarium, University of Kansas. 
Crataegus crus-galli L. - Cock-Spur Hawthorn. CH [!], CRW [!, K, G]. Native. OC, CL.

Crataegus mollis (Torr. \& A. Gray) Scheele - Downy Hawthorn. CH [K], CRW [!, K, G]. Native. OC.

Crataegus pruinosa (Wendl. f.) K. Koch - Waxy-Fruit Hawthorn. $\mathrm{CH}$ [K], CRW [!, K, G]. Native. Rare. CL. Reported in six counties in Kansas but common in surrounding states.

Crataegus punctata Jacq. - Dotted Hawthorn. CH [K], CRW [K, G]. Native. Rare. Reported in four counties in Kansas but common in surrounding states.

Crataegus succulenta Schrad. ex Link - Fleshy Hawthorn. CH [K]. Native. Rare. Reported in three counties in Kansas; common in Missouri but not reported from Oklahoma or Arkansas.

Crataegus viridis L. var. viridis - Green Hawthorn. CH [!, K], CRW [!, K, G]. Native. OC, CL. Gibson did not recognize varities of this taxon.

Fragaria virginiana Duchesne subsp. virginiana-Virginia Strawberry. $\mathrm{CH}[!, \mathrm{K}], \mathrm{CRW}[!, \mathrm{K}, \mathrm{G}]$. Native. OP, CL.

Geum canadense Jacq. var. canadense - White Avens. CH [!, K], CRW $[!, \mathrm{K}]$. Native. OP, $\mathrm{CL}$.

Geum vernum (Raf.) Torr. \& A. Gray - Spring Avens. CH [!, K], CRW [!, $\mathrm{K}, \mathrm{G}]$. Native. $\mathrm{OC}, \mathrm{CL}$.

Geum virginianum L. - Cream Avens. CH [!], CRW [!, G]. Native. Rare. OP, CL. State Record (Pryer 1719, Pryer 4958, Pryer 5364). Known from several counties in the southern half of Missouri and ten from northwestern Arkansas, but uncommon in all of these counties (Kartesz 2017).

Gillenia stipulata (Muhl. ex Willd.) Baill. - Indian-Physic. CH [!, K], CRW [K]. Native. Rare. OP. Reported in three counties in Kansas but common in surrounding states.

Malus baccata (L.) Borkh. - Siberian Crabapple. CRW [!]. Non-native. OC. Crawford County Record (Pryer 5281).

Malus ioensis (Wood) Britton - Prairie Crabapple. CH [K]. Native.

Physocarpus opulifolius (L.) Maxim. var. intermedius (Rydb.) B.L. Rob. - Atlantic Ninebark. CH [K]. Native. Rare. Reported in two counties in Kansas; common in Missouri and Arkansas but rare in Oklahoma.

Potentilla indica (Andr.) T. Wolf - Indian-Strawberry. CRW [!, K]. Non-native. CL.

Potentilla norvegica L. - Norwegian Cinquefoil. CH [!, K], CRW [!, $\mathrm{K}, \mathrm{G}]$. Native.

Potentilla recta L. - Sulphur Cinquefoil. CH [!, K], CRW [!, K, G]. Nonnative. $\mathrm{OC}, \mathrm{CL}$.

Potentilla simplex Michx. - Oldfield Cinquefoil. CH [!, K], CRW [!, K, G]. Native. OP, OC.

Prunus americana Marshall - American Plum. CH [!, K], CRW [!, K, G]. Native. CL.

Prunus angustifolia Marshall - Chickasaw Plum. CH [K], CRW [!, K, G]. Native. CL.

Prunus hortulana Bailey - Hortulan Plum. CH [!, K], CRW [!, K, G]. Native. OC, CL.

Prunus mahaleb L. - Perfumed Cherry. CH [!]. Non-native. CL. Cherokee County Record (Pryer 5478).

Prunus rivularis Scheele - Creek Plum. CH [!, K], CRW [!, K, G]. Native. $\mathrm{OC}, \mathrm{CL}$. Gibson recognized Prunus munsoniana. We are following the most recent treatment for the genus published by Flora of North America (Rohrer et al. 2014), which synonymizes $P$. munsoniana under $P$. rivularis.

Prunus serotina Ehrh. var. serotina - Black Cherry. CH [!, K], CRW [!, $\mathrm{K}, \mathrm{G}]$. Native. OP, OC, CL.

Prunus virginiana L. var. virginiana - Choke Cherry. CH [!], CRW [K, G]. Native. OP. Cherokee County Records (Pryer 139, Pryer 175, Pryer 182).

Pyrus calleryana Decne. - Bradford Pear. CH [!], CRW [!]. Non-native.
OC, CL. Cherokee County Record (Pryer 5474). Crawford County Record (Pryer 2325, Pryer 4044, Pryer 4046, Pryer 5280, Pryer 6415).

Pyrus communis L. - Common Pear. CH [!], CRW [K, G]. Non-native. OP. Cherokee County Record (Pryer 4097, Pryer 5473).

Rosa arkansana Porter - Prairie Rose. CH [!, K], CRW [!, K, G]. Native. $\mathrm{OP}, \mathrm{OC}, \mathrm{CL}$.

Rosa carolina L. subsp. carolina - Carolina Rose. CH [!, K], CRW [!, $\mathrm{K}, \mathrm{G}$. Native. OP, CL.

Rosa carolina L. subsp. subserrulata (Rydb.) W.H. Lewis - Carolina Rose. CH [!, K], CRW [!, K, G]. Native. OP, OC, CL.

Rosa foliolosa Nutt. ex Torr. \& A. Gray - White Prairie Rose. CH [K]. Native. Rare. Reported in two counties in Kansas; rare in Missouri and Arkansas but common in Oklahoma.

Rosa $\times$ medioccidentis W.H. Lewis - $\mathrm{CH}$ [K]. Native.

Rosa multiflora Thunb. ex Murr. - Rambler Rose. CH [!, K], CRW [!, K, G]. Non-native. Noxious. OC, CL.

Rosa setigera Michx. - Climbing Rose. CH [!, K], CRW [!, K, G]. Native. $\mathrm{OC}, \mathrm{CL}$.

Rosa spinosissima L. - Scotch Rose. CRW [G]. Non-native. Reported by Gibson (1963) as being established locally in Crawford County, but the only specimen at KSP (Nelson s.n. [KSP024619]) was from the Old Frisco Tree Farm in Farlington from 1950. We thus report it here for future reference but do not include it in numerical tallies.

Rubus allegheniensis Porter var. allegheniensis - Allegheny Blackberry. CH [!, K], CRW [!, K, G]. Native. OP, OC, CL. Reported from eighteen eastern counties in Kansas; more common east and (especially) north.

Rubus bushii Bailey - Bush's Blackberry. CH [!, K]. Native. OP.

Rubus flagellaris Willd. - Whiplash Dewberry. CH [!, K], CRW [!, K]. Native. OC, CL.

Rubus occidentalis L. - Black Raspberry. CH [K], CRW [!, K, G]. Native. $\mathrm{OC}, \mathrm{CL}$.

Rubus roribaccus (Bailey) Rydb. - Lucretia Dewberry. CH [K], CRW [!] Native. Crawford County Record (Pryer 4849). In Kansas known from ten central or eastern counties; elsewhere mostly east and north.

Rubus trivialis Michx. - Southern Dewberry. CH [K], CRW [!]. Native. Crawford County Record (Pryer 4413). In Kansas known only from Labette, Cherokee and Crawford counties; more common east and especially south.

RUBIACEAE (Madder Family)

Cephalanthus occidentalis L. - Common Buttonbush. CH [!, K], CRW $[!, \mathrm{K}, \mathrm{G}]$. Native. OC, CL.

Cruciata pedemontana (Bellardi) Ehrend. - Piedmont-Bedstraw. $\mathrm{CH}$ $[!, \mathrm{K}], \mathrm{CRW}[!, \mathrm{K}]$. Non-native. OP, OC, CL.

Diodia virginiana L. - Virginia Buttonweed. CH [!, K], CRW [!, K]. Native. Rare. OP. Reported in three counties in Kansas but common in surrounding states.

Galium aparine L. - Sticky-Willy. CH [!, K], CRW [!, K, G]. Native. OP, $\mathrm{OC}, \mathrm{CL}$.

Galium circaezans Michx. - Licorice Bedstraw. CH [!, K], CRW [!, K, G]. Native. OP, CL.

Galium concinnum Torr. \& A. Gray - Shining Bedstraw. CH [!, K]. Native. OP.

Galium obtusum Bigelow subsp. obtusum - Blunt-Leaf Bedstraw. $\mathrm{CH}$ [K], CRW [!, G]. Native. OC. Gibson did not recognize subspecies of this taxon.

Galium pilosum Aiton - Hairy Bedstraw. CH [!, K], CRW [K]. Native. OP, OC, CL. Crawford County Record (Pryer 1319, Pryer 1447).

Galium sherardia Krause - Blue Field-Madder. CH [!]. Non-native. OP. Cherokee County Record (Pryer 377). 
Galium triflorum Michx. - Fragrant Bedstraw. CH [!, K], CRW [!, K, G]. Native. OP, OC, CL.

Galium virgatum Nutt. - Southwestern Bedstraw. CH [K], CRW [!, $\mathrm{K}, \mathrm{G}]$. Native. OC.

Hexasepalum teres (Walt.) J.H. Kirkbr. - Poor joe. CH [!, K], CRW [!, K, G]. Native. OP, OC, CL.

Houstonia longifolia Gaertn. - Long-Leaf Summer Bluet. CH [K], CRW [!, K, G]. Native. Crawford County Record (Fish s.n. [KSP020100]) and Rare. OC. In Kansas previously reported only in Cherokee County; common in surrounding states. The cited voucher was collected in 1949 from Farlington State Park from a limestone cliff. The specimen was labeled with this taxonomic name so it is uncertain why Gibson (1963) overlooked it.

Houstonia pusilla Schoepf - Tiny Bluet. CH [!, K], CRW [!, K, G]. Native. OP, OC, $\mathrm{CL}$.

Spermacoce glabra Michx. - Smooth False Buttonweed. CH [!, K], CRW [K]. Native. Rare. CL. Reported in eight counties in Kansas but common in surrounding states.

Stenaria nigricans (Lam.) Terrell var. nigricans - Diamond-Flowers. $\mathrm{CH}[\mathrm{K}], \mathrm{CRW}[\mathrm{K}, \mathrm{G}]$. Native.

RUSCACEAE (Butcher's-Broom Family)

Maianthemum racemosum (L.) Link subsp. racemosum - Feathery False Solomon's-Seal. CH [K]. Native.

Polygonatum biflorum (Walt.) Elliott var. biflorum - King Solomon'sSeal. CH [K], CRW [!, K, G]. Native. CL.

RUTACEAE (Rue Family)

Ptelea trifoliata L. subsp. trifoliata-Common Hoptree. CH [!, K], CRW $[\mathrm{K}, \mathrm{G}]$. Native. OC, CL.

Zanthoxylum americanum P. Mill. - Toothachetree. CH [!, K], CRW [!, K, G]. Native. CL.

SALICACEAE (Willow Family)

Populus alba L. - White Poplar. CH [K], CRW [!, K, G]. Non-native. CL. Cherokee County Record (Pryer 5471).

Populus deltoides W. Bartram ex Marshall - Eastern Cottonwood. CRW [G] Gibson did not recognize subspecies of this taxon at the time of his publication. Only the taxon with infraspecific designations are included in the overall tallies for the counties.

Populus deltoides W. Bartram ex Marshall subsp. deltoides - Eastern Cottonwood. CRW [!]. Native. CL. State Record. Crawford County voucher (Pryer 781). Reported throughout the eastern United States, including the 4-state region, and occurring in several counties bordering Kansas in Oklahoma and Missouri.

Populus deltoides W. Bartram ex Marshall subsp. monilifera (Aiton) Eckenwalder - Eastern Cottonwood. CH [!, K], CRW [!, K, G]. Native. CL.

Salix amygdaloides Anderss. - Peach-Leaf Willow. CH [K], CRW [K, G]. Native.

Salix caroliniana Michx. - Carolina Willow. CH [K], CRW [K, G]. Native. Salix eriocephala Michx. - Missouri Willow. CH [!]. Native. CL. Cherokee County Record (Pryer 479, Pryer 1199, Pryer 5777).

Salix famelica (C.R. Ball) Argus - CRW [K]. Native.

Salix humilis Marshall var. humilis - Prairie Willow. CH [K], CRW [G]. Native.

Salix interior Rowlee - Sandbar Willow. CH [!, K], CRW [!, K, G]. Native. OC, CL.

Salix nigra Marshall - Black Willow. CH [!, K], CRW [!, K, G]. Native. $\mathrm{OC}, \mathrm{CL}$.

SALVINIACEAE (Water Fern Family)

Azolla microphylla Kaulfuss - Mexican Mosquito Fern. CH [!, K]. Native. OP.

SANTALACEAE (Sandalwood Family)

Comandra umbellata (L.) Nutt. subsp. umbellata-Bastard-Toadflax. $\mathrm{CH}[\mathrm{K}]$. Native.
Phoradendron leucarpum (Raf.) Reveal \& M.C. Johnst. subsp. leucarpum - Oak Mistletoe. CH [K], CRW [K]. Native. Rare. Reported in six counties in Kansas; common in surrounding states. In Missouri this taxon has a southern distribution.

SAPINDACEAE (Soapberry Family)

Aesculus glabra Willd. var. arguta (Buckl.) B.L. Rob. - Ohio Buckeye. $\mathrm{CH}[\mathrm{K}], \mathrm{CRW}[\mathrm{K}, \mathrm{G}]$. Native.

Aesculus glabra Willd. var. glabra - Ohio Buckeye. CRW [!]. Native. Rare. OC, CL. Crawford County Record (Pryer 2052, Pryer 2726, Pryer 2727, Pryer 2728, Pryer 2729, Pryer 2789, Pryer 4354). Reported in two counties in Kansas but common in surrounding states.

Sapindus saponaria L. var. drummondii (Hook. \& Arn.) L. Benson Wing-Leaf Soapberry. CRW [!, G]. Native. CL.

SAPOTACEAE (Sapodilla Family)

Sideroxylon lanuginosum Michx. subsp. oblongifolium (Nutt.) T.D. Pennington - Gum Bully. CH [!, K], CRW [!, K, G]. Native. OC, CL.

SAURURACEAE (Lizard's-Tail Family)

Saururus cernuus L. - Lizard's-Tail. CH [K]. Native. Rare. Reported in two counties in Kansas but common in surrounding states.

SAXIFRAGACEAE (Saxifrage Family)

Heuchera americana L. var. hirsuticaulis (Wheelock) Rosend., Butters, \& Lakela - American Alumroot. CH [K]. Native.

Heuchera richardsonii R. Br. - Richardson's Alumroot. CH [!, K]. Native. OP.

SCROPHULARIACEAE (Figwort Family)

Scrophularia lanceolata Pursh - Lance-Leaf Figwort. CH [!, K]. Native. OP.

Scrophularia marilandica L. - Carpenter's-Square. CH [!, K], CRW [!, $\mathrm{K}, \mathrm{G}]$. Native. OP, OC, CL.

Verbascum blattaria L. - White Moth Mullein. CH [!, K], CRW [!, K, G]. Non-native. OP, CL.

Verbascum thapsus L. - Great Mullein. CH [!, K], CRW [!, K, G]. Nonnative. $\mathrm{OP}, \mathrm{OC}, \mathrm{CL}$.

SIMAROUBACEAE (Quassia-Wood Family)

Ailanthus altissima (P. Mill.) Swingle - Tree-of-Heaven. CRW [K, G]. Non-native.

SMILACACEAE (Greenbrier Family)

Smilax bona-nox L. var. bona-nox - Fringed Greenbrier. CH [!, K], CRW [!, K, G]. Native. OC, CL.

Smilax ecirrata (Engelm. ex Kunth) S. Wats. - Upright Carrion-Flower. $\mathrm{CH}[\mathrm{K}]$. Native.

Smilax glauca Walt. - Sawbrier. CRW [!, G]. Native. OC, CL. State Record (Pryer 5218).

Smilax hispida Muhl. ex Torr. - Chinaroot. CH [!, K], CRW [!, K, G]. Native. OC, CL.

Smilax lasioneuron Hook. - Blue Ridge Carrion-Flower. CH [K], CRW [K]. Native.

Smilax pulverulenta Michx. - Downy Carrion-Flower. CH [K]. Native. Rare.

SOLANACEAE (Potato Family)

Datura stramonium L. - Jimsonweed. CH [!, K], CRW [!, K, G]. Nonnative. CL.

Lycium barbarum L. - Matrimony-Vine. CRW [!]. Non-native. OC. Crawford County Record (Pryer 3588).

Petunia axillaris (Lam.) B.S.P. - Garden Petunia. CH [K]. Non-native.

Physalis angulata L. - Cut-Leaf Ground-Cherry. CH [!, K], CRW [K]. Native. OP, OC, CL. Crawford County Record (Pryer 1677, Pryer 1873, Pryer 5766, Pryer 5932).

Physalis heterophylla Nees - Clammy Ground-Cherry. CH [K], CRW [!, K, G]. Native. CL. 
Physalis longifolia Nutt. - Long-Leaf Ground-Cherry. CRW [!, K, G]. Native. Gibson did not recognize varieties of this taxon. Only one specimen was found and reidentfied as $P$. longifolia var. subglabrata. It is expected that specimens reported by Gibson probably represented both varieties. Only the taxon including varieties are used in tallies for each county.

Physalis longifolia Nutt. var. longifolia - Long-Leaf Ground-Cherry. $\mathrm{CH}[!, \mathrm{K}], \mathrm{CRW}[!, \mathrm{K}]$. Native. OP. CL.

Physalis longifolia Nutt. var. subglabrata (Mack. \& Bush) Cronquist Long-Leaf Ground-Cherry. CH [!, K], CRW [!, K, G]. Native. OC, CL.

Physalis missouriensis Mack. \& Bush - Missouri Ground-Cherry. CRW [!], CRW [K]. Native. OC. Crawford County Record (Pryer 5585). Physalis pubescens L. - Husk-Tomato. CH [!, K], CRW [K, G]. Native. OP. Physalis pumila Nutt. var. pumila - Dwarf Ground-Cherry. CH [!, K], CRW [!, K, G]. Native. OP, OC, CL. Gibson did not recognize varieties of this taxon.

Physalis virginiana P. Mill. var. virginiana - Virginia Ground-Cherry. CH [!, K], CRW [!]. Native. CL. Crawford County Record (Pryer 532, Pryer 778, Pryer 2546).

Solanum americanum P. Mill. - American Black Nightshade. CRW [!, G]. CL. State Record (Pryer 6150).

Solanum carolinense $\mathrm{L}$. var. carolinense - Carolina Horse-Nettle. $\mathrm{CH}$ $[!, K]$, CRW [!, K, G]. Native. Noxious. OP, OC, CL. Gibson did not recognize varities of this taxon.

Solanum dimidiatum Raf. - Western Horse-Nettle. CH [!, K], CRW [!, G]. Native. Rare. OC, CL. Reported in nine counties in Kansas; common in Oklahoma with occasional reports for Missouri and Arkansas, where it is considered adventive.

Solanum elaeagnifolium Cav. - Silver-Leaf Nightshade. Ch [K]. Native. Noxious.

Solanum ptychanthum Dunal - Eastern Black Nightshade. CH [!, K], CRW [!, K]. Native. Noxious. OC, CL.

Solanum rostratum Dunal - Horned Nightshade. CH [!, K], CRW [!, $\mathrm{K}, \mathrm{G}$. Native. OC, $\mathrm{CL}$.

Solanum sarrachoides Sendtn. - Tropical American Nightshade. $\mathrm{CH}$ [!], CRW [!]. Non-native. OP, OC, CL. State Record (Pryer 1544, Pryer 1947, Pryer 2397, Pryer 3587, Pryer 6013).

STAPHYLEACEAE (Bladdernut Family)

Staphylea trifolia L. - American Bladdernut. CH [!, K], CRW [!, K, G]. Native. OP, OC, CL.

TRILLIACEAE (Trillium Family)

Trillium sessile L. - Toadshade. CH [K], CRW [!, K, G]. Native. OP, OC, CL. Trillium viridescens Nutt. - Taper-Tip Trillium. CH [!, K], CRW [K]. Native. Rare. OP. Only reported in Cherokee County, Kansas, but common in surrounding states.

TYPHACEAE (Cat-Tail Family)

Typha angustifolia L. - Narrow-Leaf Cat-Tail. CH [K], CRW [!, K, G]. Non-native. CL.

Typha domingensis Pers. - Southern Cat-Tail. CH [!, K]. Native. CL.

Typha latifolia L. - Broad-Leaf Cat-Tail. CH [!, K], CRW [!, K, G]. Native. CL.

ULMACEAE (Elm Family)

Ulmus alata Michx. - Winged Elm. CH [K]. Native. Rare. Only reported in Cherokee County, Kansas, but common in surrounding states.

Ulmus americana L. - American Elm. CH [!, K], CRW [!, K, G]. Native. $\mathrm{OP}, \mathrm{OC}, \mathrm{CL}$.

Ulmus $\times$ intermedia Elowsky $-\mathrm{CH}[\mathrm{K}]$.

Ulmus parvifolia Jacq. - Chinese Elm. CRW [!, K]. Non-native. CL. Ulmus pumila L. - Siberian Elm. CH [K], CRW [!, K, G]. Native. OC. Ulmus rubra Muhl. - Slippery Elm. CH [!, K], CRW [!, K, G]. Native. $\mathrm{OC}, \mathrm{CL}$.
URTICACEAE (Nettle Family)

Boehmeria cylindrica (L.) Sw. - Small-Spike False Nettle. CH [!, K], CRW [!, K, G]. Native. OP, OC, CL.

Laportea canadensis (L.) Wedd. - Canadian Wood-Nettle. CH [!, K], CRW [!, K, G]. Native. OP, OC, CL.

Parietaria pensylvanica Muhl. ex Willd. - Pennsylvania Pellitory. $\mathrm{CH}$ [!], CRW [!, K, G]. Native. OP, OC, CL.

Pilea pumila (L.) A. Gray - Canadian Clearweed. CH [!, K], CRW [!, K, G]. Native. OP, OC, CL.

Urtica chamaedryoides Pursh - Heart-Leaf Nettle. CH [K]. Native. Rare. Reported in three counties in Kansas; common in the surrounding areas. Rare in Missouri and Oklahoma but common in Arkansas.

Urtica dioica L. subsp. gracilis (Aiton) Seland. - Stinging Nettle. $\mathrm{CH}[\mathrm{K}]$. Native.

VALERIANACEAE (Valerian Family)

Valerianella radiata (L.) Dufr. - Beaked Cornsalad. CH [!, K], CRW [!, $\mathrm{K}, \mathrm{G}]$. Native. OP, CL.

VERBENACEAE (Verbena Family)

Glandularia bipinnatifida (Nutt.) Nutt. - Dakota Mock Vervain. $\mathrm{CH}$ [!], CRW [!, K, G]. Native. OC, CL. Cherokee County Record (Pryer 4399, Pryer 4409).

Glandularia canadensis (L.) Nutt. - Rose Mock Vervain. CH [!, K], CRW [!, K, G]. Native. OP, OC, CL.

Phyla cuneifolia (Torr.) Greene - Wedgeleaf. CH [K]. Native.

Phyla lanceolata (Michx.) Greene - Northern Frogfruit. CH [!, K], CRW [!, K, G]. Native. OC, CL.

Verbena $\times$ blanchardii Moldenke - $\mathrm{CH}[\mathrm{K}]$. Native.

Verbena bracteata Cav. ex Lag. \& Rodr. - Carpet Vervain. CH [K], CRW [!, K, G]. Native. OC.

Verbena $\times$ engelmannii Moldenke - CRW [K]. Native.

Verbena hastata L. - Simpler's-Joy. CH [!, K], CRW [!, K, G]. Native. $\mathrm{OP}, \mathrm{CL}$.

Verbena $\times$ illicita Moldenke $-\mathrm{CH}[\mathrm{K}]$. Native.

Verbena $\times$ rydbergii Moldenke $-\mathrm{CH}[\mathrm{K}]$. Native.

Verbena simplex Lehm. - Narrow-Leaf Vervain. CH [!, K], CRW [!, K, $\mathrm{G}$. Native. $\mathrm{OC}, \mathrm{CL}$.

Verbena stricta Vent. - Hoary Vervain. CH [K], CRW [!, K, G]. Native. OC. Verbena urticifolia L. - White Vervain. CH [!, K], CRW [!, K, G]. Native. $\mathrm{OC}, \mathrm{CL}$.

VIOLACEAE (Violet Family)

Cubelium concolor (T.F. Forst.) Raf. ex. Britt. \& A. Br. - Green-Violet. CH [K]. Native. Rare. In Kansas known from seven eastern counties; more common eastwards.

Viola affinis vel. af. Le Conte - Sand Violet. CRW [!]. Native. CL. Potential State Record (Pryer 17, Pryer 96). Rare in Missouri and occasional but common in Arkansas; mostly in the eastern United States.

Viola bicolor Pursh - Field Pansy. CH [!, K], CRW [!, K, G]. Native. $\mathrm{OP}, \mathrm{OC}, \mathrm{CL}$.

Viola missouriensis Greene - Missouri Violet. CH [!, K], CRW [!, G]. Native. OP, OC, CL.

Viola nephrophylla Greene - Northern Bog Violet. CH [!, K], CRW [!, $\mathrm{K}, \mathrm{G}]$. Native. OP, CL.

Viola palmata L. var. palmata -Three-Lobe Violet. CH [!, K], CRW [K]. Native. Rare. OP, OC. Crawford County Record (Pryer 4437, Pryer 4442, Pryer 4485). Reported in four counties in Kansas, including this report; common in Arkansas and Missouri but rare in Oklahoma.

Viola pedata L. - Bird-Foot Violet. CH [!, K], CRW [G]. Native. OP.

Viola pedatifida G. Don - Crow-Foot Violet. CH [K], CRW [K, G]. Native. Viola pubescens Aiton - Downy Yellow Violet. CH [!, K], CRW [!, K, G]. Native. $\mathrm{OC}, \mathrm{CL}$. 
Viola sagittata Aiton var. sagittata - Arrow-Leaf Violet. CH [!, K], CRW [!, K, G]. Native. OP, OC. Gibson did not recognize varieties of this taxon.

Viola sororia Willd. - Hooded Blue Violet. CH [!, K], CRW [!, K, G]. Native. $\mathrm{OP}, \mathrm{OC}, \mathrm{CL}$

Viola striata Aiton - Striped Cream Violet. CRW [G]. Native. Reported by Gibson (1963) as "Sperry 1962, established."The only specimen at KSP is Sperry P-252 ([KSP014237]), which is from Sperry's residence in 1963, and which indicated "escaped." Many hundreds of specimens at KSP originate from Sperry's "Paradocs" (the name of his small acreage in Pittsburg), but the nativity of all must be considered suspect. Thus while noted here, this species is not included in numerical summaries.

Viola subsinuata Greene - Early Blue Violet. CH [K]. Native.

Viola $\times$ viarum Pollard $-\mathrm{CH}[\mathrm{K}]$. Native.

VITACEAE (Grape Family)

Ampelopsis cordata Michx. - Heart-Leaf Peppervine. CH [!, K], CRW $[!, \mathrm{K}, \mathrm{G}]$. Native. OC, CL.
Cissus trifoliata (L.) L. - Sorrelvine. CH [K]. Native. Rare. Reported in three counties in Kansas and also rare in Missouri; common in Oklahoma and Arkansas.

Parthenocissus inserta (Kerner) Fritsch - Thicket-Creeper. CRW [!]. Native. OC. Crawford County Record (Pryer 2771).

Parthenocissus quinquefolia (L.) Planch. - Virginia-Creeper. CH [!, K], CRW [!, K, G]. Native. OP, CL.

Vitis aestivalis Michx. var. aestivalis - Summer Grape. CH [!, K], CRW [!, G]. Native. OP, CL.

Vitis cinerea (Engelm.) Engelm. ex Millard var. cinerea - Gray-Bark Grape. CH [!, K], CRW [!, K, G]. Native. OP, OC, CL. Gibson did not recognize varieties of this taxon.

Vitis riparia Michx. - River-Bank Grape. CH [!, K], CRW [!, K, G]. Native. OC, CL.

Vitis vulpina L. - Frost Grape. CH [!, K], CRW [!, K, G]. Native. OP, OC, CL.

ZYGOPHYLLACEAE (Creosote-Bush Family)

Tribulus terrestris L. - Puncturevine. CH [K], CRW [K, G]. Non-native.

\section{DEDICATION}

We dedicate this study to the memory of Dr. Ronald L. Hartman (1945-2018), MS advisor to the second author and long-time Director of the Rocky Mountain Herbarium at the University of Wyoming. His 93,000+ collections and mentorship of over fifty graduate students in floristics spanning nearly forty years significantly increased knowledge of plant distributions in the Rocky Mountains and neighboring regions.

\section{ACKNOWLEDGMENTS}

Many thanks to the landowners who allowed access to their properties and those who facilitated contact with local landowners, in particular Janice Harryman. Special thanks to Cindy and John Epler, John Foster, Glenn Salsbury, and Bill Reid who helped the first author locate properties for research. Without these individuals, this study would never have been possible. Thanks to the Missouri Botanical Garden for use of their Missouri and Illinois Reference Collection as well as the space and tools to help confirm identifications, in particular Jim Solomon and Mary McNamara for facilitating the visits. John Pruski and Ron Leisner at MO helped identify specimens. At KANU we appreciate Craig Freeman and Caleb Morse for allowing us to report their state record specimens and for providing access to the McGregor Herbarium; Caleb also compiled a list of specimens located in McGregor Herbarium for Crawford and Cherokee counties. Thanks to Chance Curran for sharing data on a grass specimen. Jiawei Xu (KSP) kindly translated the abstract into Chinese. We appreciate the numerous constructive comments of Mark Mayfield (KSC), who also kindly annotated Euphorbiaceae at KSP, and those of an anonymous reviewer. A final thank you to American Society of Plant Taxonomists for a student scholarship to the first author for helping to fund this study.

\section{REFERENCES}

Angelo, R.T., M.S. Cringan, D.L. Chamberlal, A.J. Stahl, S.G. Haslouer, \& C.A. Goodrich. 2007. Residual effects of lead and zinc mining on freshwater mussels in the Spring River Basin (Kansas, Missouri, and Oklahoma, USA). Sci. Total Environm. 384:467-496.

APG IV. 2016. An update of the Angiosperm Phylogeny Group classification for the orders and families of flowering plants: APG IV. Bot. J. Linnaean Soc. 181:1-20.

BAiley, T. \& C. Hooey. 2017. "From wasteland to wonderland:" New uses for mined land in rural southeast Kansas. The Midwest Quarterly 58(3):252-263.

Barker, W.T., T.M. Barkley, \& Great Plains Flora Association. 1977. Atlas of the flora of the Great Plains. lowa State Press, Ames, U.S.A.

BARKLEY, T.M. 1968. Manual of the flowering plants of Kansas. Kansas Agricultural Experiment Station, U.S.A.

BAsS, H.E. 1949. Prairie flora of K.S.T.C. campus at Pittsburg, Crawford County, Kansas. M.S. thesis, Kansas State Teachers College (= Pittsburg State University), Pittsburg, Kansas, U.S.A. 
Bebber, D.P., M.A. Carine, J.R.I. Wood, A.H. Wortley, D.J. Harris, G.T. Prance, G. Davidse, J. Paige, T.D. Pennington, N.K.B. Robson, \& R.W. Scotland. 2010. Herbaria are a major frontier for species discovery. Proc. Natl. Acad. Sci., USA 107(51):22169-22171.

Bebber, D.P., M.A. Carine, G. Davidse, D.J. Harris, E.M. Haston, M.G. Penn, S. Cafferty, J.R.I. Wood, \& R.W. Scotland. 2012. Big hitting collectors make massive and disproportionate contributions to the discovery of plant species. Proc. Royal Soc. B 279:2269-2274.

Beyer, W.N., J. Dalgarn, S. Dudding, J.B. French, R. Mateo, J. Miesner, L. Sileo, \& J. Spann. 2004. Zinc and lead poisoning in wild birds in the tri-state mining district (Oklahoma, Kansas, and Missouri). Arch. Environm. Contam. Toxicol. 48(1):108-117.

BRADY, L.L. 2018. Mining history in Kansas. Federal Highway Administration. U.S. Department of Transportation, Washington, DC, U.S.A.

BuchanAn, R. 2010. Kansas geology: An introduction to landscapes, rocks, minerals, and fossils. 2nd ed. University Press of Kansas, Lawrence, Kansas, U.S.A.

Buerki, S., P.P. Lowry II, N. Alvarez, S.G. Razafimandimbison, P. Küpfer, \& M.W. Callmander. 2010. Phylogeny and circumscription of Sapindaceae revisited: Molecular sequence data, morphology and biogeography support recognition of a new family, Xanthoceraceae. PI. Ecol. Evol. 143(2):148-159.

Chapman, A.D. 2005. Uses of primary species-occurrence data, version 1.0. Report for the Global Biodiversity Information Facility, Copenhagen, Denmark.

Chapman, S.S., J.M. Omernik, J.A. Freeouf, D.G. Huggins, J.R. McCauley, C.C. Freeman, G. Steinauer, R.T. Angelo, \& R.L. Schlepp. 2001. Ecoregions of Nebraska and Kansas (color poster with map, descriptive text, summary tables, and photographs): Reson, Virginia, U.S. Geological Survey (map scale 1:1,950,000).

Charboneau, J.L.M. 2013. A floristic inventory of Valley and Phillips counties, Montana, U.S.A. M.Sc. thesis, Univ. of Wyoming, Laramie, U.S.A.

CLAY, K. 1990. Fungal endophytes of grasses. Ann. Rev. Ecol. Syst. 21:275-297.

CULLEY, T.M. 2013. Why vouchers matter in botanical research. Appl. PI. Sci. 1(11):1300076.

CULLEY, T.M. \& N.A. HARDIMAN. 2007. The beginning of a new invasive plant: A history of the ornamental callery pear in the United States. BioScience 57(11):956-964.

Daru, B.H., D.S. Park, R.B. Primack, C.G. Willis, D.S. Barrington, T.J.S. Whitfeld, T.G. Seidler, P.W. Sweeney, D.R. Foster, A.M. Ellison, \& C.C. DAvIs. 2017. Widespread sampling biases in herbaria revealed from large-scale digitization. New Phytologist 217:939-955.

DIGGS, G.M., JR., B.L. LIPSCOMB, \& R.J. O'KENNON. 1999. Shinners and Mahler's illustrated flora of North Central Texas. Botanical Research Institute of Texas Press, Fort Worth, Texas, U.S.A.

ERTTER, B. 2000. Floristic surprises in North America north of Mexico. Ann. Missouri Bot. Gard. 87:81-109.

FreCKmAnN, R.W. \& M.G. Lelong. 2003. Dichanthelium. In: Flora of North America north of Mexico, Vol. 25. Oxford Press, New York, U.S.A. Pp. 405-450.

Funk, V. 2004. 100 uses for an herbarium (well at least 72). Division of Botany, Yale University Herbarium, New Haven, Connecticut, U.S.A.

GATES, F.C. 1936. Grass in Kansas. Rep. Kansas State Board Agric. 45(204-B). Topeka, Kansas, U.S.A.

GATES, F.C. 1940. Flora of Kansas. Agric. Exp. Stat., Contr. No. 391. Kansas State College of Agriculture and Applied Science, Topeka, Kansas, U.S.A.

GBIF (Global Biodiversity Information Facility). 2018. What is GBIF? www.gbif.org/what-is-gbif.

GiBson, E.S. 1963. Vascular flora of Crawford County, Kansas. Trans. Kansas Acad. Sci. 66(4):684-726.

GIER, L.J. 1931. A preliminary key to the herbaceous dicotyledons of the Cherokee Strip of southeast Kansas. Trans. Kansas Acad. Sci. 34:255-267.

Gillespie, L.J., J.M. SAARELA, P.C. Sokoloff, \& R.D. Bull. 2017. New vascular plant records for the Canadian Arctic Archipelago. PhytoKeys 52:23-79.

GPFA (Great Plains Flora Association). 1977. Atlas of the flora of the Great Plains. lowa State University Press, Ames, U.S.A. GPFA (Great Plains Flora Association). 1986. Flora of the Great Plains. University Press of Kansas, Lawrence, Kansas. U.S.A. Grant, V. 1981. Plant speciation. Columbia University Press, New York, U.S.A.

Gries, C., E.E. GILber, \& N.M. Franz. 2014. Symbiota - A virtual platform for creating voucher-based biodiversity information communities. Biodiv. Data J. 2:e1114. doi: 10.3897//BDJ.2.e1114

Hartman, R.L. \& B.E. Nelson. 1998. Taxonomic novelties from North America north of Mexico: A 20-year vascular plant diversity baseline. Monogr. Syst. Bot., Missouri Bot. Gard. 67:1-59. 
HAZLETT, D.L. 2004. Vascular plant species of the Comanche National Grassland in southeastern Colorado. U.S.D.A. Forest Service. Gen. Tech. Rep. RMRS-GTR-130.

Hodkinson, I.D. 1984. Chapter 3: The biology and ecology of the gall-formaing Psylloidea (Homoptera). In: T.N. Ananthakrishnan, ed. Biology of Galls. Oxford \& IBH Publishing Co., New Delhi, Bombay, Calcutta, India. Pp. 59-77.

James, S.A., P.S. Soltis, L. Belbin, A.D. Chapman, G. Nelson, D.L. Paul, \& M. Collins. 2018. Herbarium data: Global biodiversity and societal botanical needs for novel research. Appl. PI. Sci. 6(2):e1024.

Kansas Department of Health and Environment. Abandoned Mine Land Program. www.kdheks.gov/mining/abandoned_ mineland.html. Accessed May 2018.

Kansas Department of Health and Environment. Regulatory Program. www.kdheks.gov/mining/regulatory_program.html. Accessed May 2018.

Kansas Department of Wildlife Parks and Tourism. Mined Land Wildlife Area Pamphlet. https://ksoutdoors.com/Services/ Publications. Accessed May 2018.

KARTESZ, J. 2017. The Biota of North America's Floristic Synthesis of North America. Beta version (unpublished). Univ. of North Carolina Botanical Garden, Chapel Hill, North Caroina, U.S.A.

Kaul, R.B., D. Sutherland, \& S. Rolfsmeier. 2011. The flora of Nebraska. Second edition. School of Natural Resources, University of Nebraska, Lincoln, Nebraska, U.S.A.

KILPATRICK, R.A., A.E. RICH, \& J.G. ConkLIN. 1961. Juncus effusus, a new host for Epichloe typhina. PI. Dis. Reporter 45:899.

KIRKPATRICK, R. 1973. The Vitaceae of southeast Kansas. M.Sc. thesis. Pittsburg State University, Pittsburg, Kansas, U.S.A.

Larson, J., B. Reif, B.E. Nelson, \& R.L. Hartman. 2014. Floristic studies in north central New Mexico, U.S.A., the Sangre de Cristo Mountains. J. Bot. Res. Inst. Texas 8(1):271-303.

LAVOIE, C. 2013. Biological collections in an ever changing world: Herbaria as tools for biogeographical and environmental studies. Perspect. PI. Ecol. Evol. Syst. 15:68-76.

LEGLER, B.S. 2008. A floristic inventory of Vermejo Park Ranch, New Mexico and Colorado. M.Sc. thesis, University of Wyoming, Laramie, Wyoming, U.S.A.

LEGLER, B.S. 2010. Additions to the vascular flora of New Mexico. J. Bot. Res. Inst. Texas 4:777-784.

LoRING, H., K. KInDSCher, \& J. DelisLe. 2005. A survey of natural area sites in Cherokee County, Kansas. Kansas Natural Heritage Inventory, U.S.A.

MAJURE, L.M., W.S. JUdD, P.S. SoltIS, \& D.E. SoltIs. 2017. Taxonomic revision of the Opuntia humifusa complex (Opuntieae: Cactaceae) of the eastern United States. Phytotaxa 290(1):001-065.

MAnders, G.C. \& J.S. Aber. 2014. Tri-State mining district legacy in northeastern Oklahoma. Emporia State Res. Stud. 49(2):29-51.

MARTINE, C.T. \& M.E. WARD. 2013. Establishment of regional herbarium leads to more than 200 new flora atlas records for New York. J. Torrey Bot. Soc. 140:125-131.

MCCAULEY, J.R., L.L. BRADY, \& F.W. WILSON. 1983. A study of the stability problems and hazard evaluation of the Kansas portion of the tri-state mining area. Kansas Geol. Surv., Open-file Rep. 83(2):193.

van der Merwe, D., J.W. CarpenteR, J.C. Nietfeld, \& J.F. Miesner. 2011. Adverse health effects in Canada geese (Branta canadensis) associated with waste from zinc and lead mines in the tri-state mining district (Kansas, Oklahoma, and Missouri, USA). J. Wildlife Dis. 47(3):650-660.

Mosyakin, S.L. \& K.R. RoberTson. 2003. Amaranthus. In: Flora of North America, Vol. 4. Oxford University Press, New York, U.S.A. Pp. $410-425$.

NCDC-CDO (National Climatic Data Center-Climate Data Online). 2018. National Centers for Environmental Information. National Oceanic and Atmospheric Administration. www.ncdc.noaa.gov/cdo-web/.

Nelson, E.T. 1952. A taxonomic and ecological study of the St. Louis - San Francisco Tree Farm, Farlington, Crawford County, Kansas. M.S. thesis, Kansas State Teachers College (= Pittsburg State University), Pittsburg, Kansas, U.S.A.

Nesom, G.L. 2008. Ranunculus ficaria (Ranunculaceae), naturalized in Texas. J. Bot. Res. Inst. Texas 2:741-742.

Nesom, G.L. 2015. Ranunculus ficaria (Ranunculaceae) naturalized in Texas: Update. Phytoneuron 27:1-7.

Palmer, M.W. \& J.C. Richardson. 2012. Biodiversity data in the information age: Do $21^{\text {st }}$ century floras make the grade? Castanea 77(1):46-59.

PATCH, E.M. 1916. A psyllid gall on Juncus (Livia Maculipennis Fitch). Maine Agric. Exp. Sta. Entomology 82:21-23.

Pavilck, L.E. \& L.K. Anderton. 2007. Bromus L. In: Flora of North America, Vol. 24. Oxford University Press, New York, U.S.A. Pp. 193-237.

Peterson, P.M. 2003. Muhlenbergia. In: Flora of North America, Vol. 25. Oxford University Press, New York, U.S.A. Pp. 145-200. 
Post, A.R., A. KRINGS, W.A. WALL, \& J.C. NeAL. 2009. Introduced lesser celandine (Ranunculus ficaria, Ranunculaceae) and its putative subspecies in the United States: A morphometric analysis. J. Bot. Res. Inst. Texas 3:193-209.

Prather, A., A.O. Alvarez-Fuentes, M.H. Mayfield, \& C.J. Ferguson. 2004a. The decline of plant collecting in the United States: A threat to the infrastructure of biodiversity studies. Syst. Bot. 29(1):15-28.

Prather, A., A.O. Alvarez-Fuentes, M.H. Mayfield, \& C.J. Ferguson. 2004b. Implications of the decline of plant collecting for systematic and floristic research. Syst. Bot. 29(1):216-220.

PYKe, G.H. \& P.R. EhrLich. 2010. Biological collections and ecological/environmental research: A review, some observations and a look to the future. Biol. Rev. 85:247-266.

QueEnBorough, S. 2017. Collections-based studies of plant functional traits. In: I. Friis \& H. Balslev, eds. Tropical plant collections: Legacies of the past? Essential tools for the future? Proc. Internat. Symp. held at the Royal Danish Academy of Sciences and Letters in Copehenhagen, 19-21 May 2015. Scientia Danica, Ser. B, Biol. 6:223-236.

Ríos-Saldaña, C.A., M. Delibes-Mateos, \& C.C. Ferreira. 2018. Are fieldwork studies being relegated to second place in conservation science? Global Ecol. Conservation 14: e00389. https://doi.org/10.1016/j.gecco.2018.e00389.

RohreR, J.R. 2014. Prunus rivularis. In: Flora of North America, Vol. 24. Magnoliphyta: Picramniaceae to Rosaceae. Oxford University Press, New York, U.S.A. Pp. 382.

Ross, B. \& H.H. Hall. 1939. A preliminary survey of the flora of Crawford County State Park, Kansas. Trans. Kansas Acad. Sci. 42:157-164.

Schiebout, M.H., D.L. Hazlett, \& N. Snow. 2008. A floristic survey of vascular plants over parts of northeastern New Mexico. J. Bot. Res. Inst. Texas 2(2):1407-1447.

SnOw, N. 1997. Application of the phylogenetic species concept: A botanical monographic perspective. Austrobaileya 5:1-8.

Snow, N., S. Young, C. Curran, \& J. Kartesz. 2017. Vascular plant survey of the Neosho Bottoms Management Area (Grand River Dam Authority) in Ottawa County, Oklahoma. Phytoneuron 26:1-27.

THIERS, B. 2019 [and continuously updated]. Index Herbariorum: A global directory of public herbaria and associated staff. New York Botanical Garden's Virtual Herbarium. http://sweetgum.nybg.org/ih/.

ThomAs, J.R. 2015. Revision of Dichanthelium sect. Lanuginosa (Poaceae). Phytoneuron 50:1-58.

USFWS (United States Fish and Wildlife Service). 2003. Mead's milkweed (Asclepias meadii) recovery plan. U.S. Fish and Wildlife Service, For Snelling, Minnesota, U.S.A.

Web Soll Survey Staff. 2018. Web Soil Survey. Natural Resources Conservation Service, United States Department of Agriculture. websoilsurvey.sc.egov.usda.gov/.

Wieczorek, J., D. Bloom, R. Guralnick, S. Blum, M. Döring, R. Giovanni, T. Robertson, \& D. Vieglais. 2012. Darwin Core: An evolving community-developed biodiversity data standard. PLoS ONE 7(1):e29715. doi: 10.1371/journal.pone.0029715.

WIER, P.J.M. 1986. Galls of Juncus scirpoides formed by Livia maculipennis (Liviidae, Homoptera). Indiana Acad. Sci. 95:247-252.

YATSKIEVYCH, G. 1999. Steyermarks's flora of Missouri: Volume 1. Missouri Botanical Garden Press, St. Louis, Missouri, U.S.A. YATSKIEVYCH, G. 2006. Steyermarks's flora of Missouri: Volume 2. Missouri Botanical Garden Press, St. Louis, Missouri, U.S.A. YATSKIEVYCH, G. 2013. Steyermarks's flora of Missouri: Volume 3. Missouri Botanical Garden Press, St. Louis, Missouri, U.S.A. 